Números de Lê e fórmulas de Lê-Iomdine para germes de hipersuperfícies singulares

Michelle Ferreira Zanchetta 


\title{
Números de Lê e fórmulas de Lê-Iomdine para germes de hipersuperfícies singulares
}

\author{
Michelle Ferreira Zanchetta
}

Orientador: Prof. Dr. Marcelo José Saia

Dissertação apresentada ao Instituto de Ciências Matemáticas e de Computação - ICMC-USP, como parte dos requisitos para obtenção do título de Mestre em Matemática.

\section{"VERSÃO REVISADA APÓS A DEFESA"}

Data da Defesa:
Visto do Orientador:

USP - São Carlos

outubro / 2006 
Aos meus pais

Luiz e Etelvina 
"De tudo ficam três coisas:

A certeza de que estamos sempre começando, A certeza de que é preciso continuar,

E a certeza de que podemos ser interrompidos antes de terminarmos.

Fazer da interrupção um caminho novo,

Da queda um passo de dança,

Do medo uma escada,

Do sonho uma ponte,

Da procura um encontro."

E assim, terá valido a pena existir...

Fernando Sabino 


\section{Agradecimentos}

A Deus, por tudo, principalmente por mais esta conquista.

A minha família pelo constante apoio e compreensão. Aos meus queridos pais, Luiz e Etelvina, pelo amor, paciência, dedicação e por suas orações, que foram fundamentais para me fortalecer e me guiar em minhas escolhas e aos meus irmãos, Gustavo e Gabriela, pela amizade e pelo carinho.

Ao meu namorado Leandro, pelo amor e apoio, por confiar no meu caráter, na minha competência e no nosso futuro.

Ao Prof. Marcelo José Saia pela orientação, atenção, incentivo, confiança e principalmente pela amizade.

Ao Prof. Roberto Callejas Bedregal, pelos ensinamentos e colaboração, que foram fundamentais para o término dessa dissertação.

Ao Rodrigo pela ajuda com os gráficos e a Grazielle, que além de colaborar para este trabalho esteve presente nos momentos que mais precisei da sua amizade.

Aos meus colegas do mestrado, pela amizade e momentos de descontração.

A todos os professores que contribuíram para a minha chegada até aqui.

Enfim, agradeço a todos que direta ou indiretamente colaboraram para a concretização deste trabalho.

Este trabalho foi patrocinado pela Fapesp, através de bolsa de mestrado processo 04/10950-7

junto ao ICMC-USP. 


\section{Resumo}

Considerando germes de hipersuperfícies em $\mathbb{C}^{n+1}$ com conjunto singular de dimensão $s$, Massey em [14] introduz um conjunto de $(s+1)$ números chamados de números de Lê. Estes números se mostram como a generalização natural do número de Milnor para singularidades isoladas. O principal objetivo deste trabalho é mostrar como estes números são obtidos e que os números de Lê de uma hipersuperfície singular estão relacionados com os números de Lê de uma certa sequência de hipersuperfícies singulares $X_{0}, \ldots, X_{s-1}$ que se aproxima da singularidade original de tal forma que os conjuntos críticos de seus termos $X_{i}$ têm dimensão $i$. Essas relações são dadas pelas fórmulas de Lê-Iomdine. 


\section{Abstract}

For any germ of hypersurface in $\mathbb{C}^{n+1}$ with singular set of dimension $s$, Massey in [14] introduces a set of $(s+1)$ numbers called Lê numbers. These numbers are a natural generalization of the Milnor number for isolated singularity hypersurfaces. The main goal of this work is to show how to obtain these numbers and to show the Lê numbers of a singular hypersurface are related with the the Lê numbers of a sequence of singular hypersurfaces $X_{0}, \ldots, X_{s-1}$ which approach the original singularity in such a way that the critical set of each $X_{i}$ has dimension $i$. These relationship are given by the Lê-Iomdine formulas. 


\section{Sumário}

1 Preliminares 3

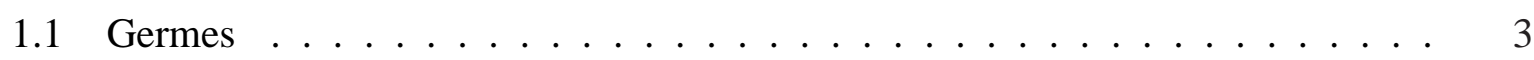

1.2 Variedades e Ideais . . . . . . . . . . . . . . . . . . 5

1.3 Espaços Analíticos Complexos . . . . . . . . . . . . . . . 7

1.4 Ciclos Analíticos . . . . . . . . . . . . . . . . . 9

2 O teorema da fibração e o número de Milnor 11

2.1 Teorema da fibração . . . . . . . . . . . . . . . . . . . . . . 11

2.2 Teorema da fibração de Lê . . . . . . . . . . . . . . . . 15

2.3 O número de Milnor . . . . . . . . . . . . . . . . . 15

3 Generalizando o Número de Milnor 17

3.1 Ciclos polares . . . . . . . . . . . . . . . . . 17

3.2 Ciclos de Lê e números de Lề . . . . . . . . . . . . . . . . . . . 21

3.3 Propriedades dos ciclos de Lề . . . . . . . . . . . . . . . . 26

3.4 Exemplos . . . . . . . . . . . . . . . . . . . . . . . . 29

3.5 Números de Lê e seções hiperplanas . . . . . . . . . . . . . . . . . . . 32

3.6 Coordenadas pré-polares . . . . . . . . . . . . . . 37

4 Fórmulas de Lê-Iomdine 44

4.1 Fórmula de Plücker e característica de Euler . . . . . . . . . . . . . . . . . . 53

4.2 Uso do Singular no cálculo dos números de Lê de um polinômio . . . . . . . . 58

4.3 Fórmulas de Lê-Iomdine generalizadas . . . . . . . . . . . . . . . . 62

$\begin{array}{ll}\text { Bibliografia } & 66\end{array}$ 


\section{Introdução}

O estudo da topologia de germes de hipersuperfícies em $\mathbb{C}^{n}$ se constitui em um dos mais importantes tópicos da teoria de singularidades e para o caso de hipersuperfícies com singularidades isoladas, o trabalho de Milnor [16] aparece com destaque. Neste é mostrado que os germes de hipersuperfícies obtidas como imagem inversa de 0 por germes de funções holomorfas $f:\left(\mathbb{C}^{n+1}, 0\right) \rightarrow(\mathbb{C}, 0)$ tem uma estrutura de fibrado, e um importante invariante numérico associado a esta estrutura de fibrado é obtido, o número de Milnor.

Desde então, o caso de hipersuperfícies com conjunto singular não isolado tem sido estudado por vários pesquisadores e dentre estes, os trabalhos de Lê, Ramanujam, Saito e Massey se destacam. Lê em [12] descreve a homologia da fibra de Milnor, Lê e Ramanujam em [10] mostram a independência do tipo de homotopia da fibra de Milnor e Lê e Saito em [11] descrevem as condições de Thom para estes germes de hipersuperfícies.

Massey em [14] generaliza o conceito de número de Milnor para germes de hipersuperfícies com conjunto singular de dimensão $s$. Associado à fibra de Milnor deste germe, ele introduz um conjunto de $s+1$ números, chamados de números de Lê.

Um dos principais objetivos deste trabalho é mostrar como estes números são obtidos.

Além disso, Massey mostra como associar uma sequência de $s$ hipersuperfícies $X_{i}$, sendo cada $X_{i}$ com conjunto singular de dimensão $i$, para $i=0, \ldots, s-1$ e relaciona os números de Lê da hipersuperfície original com os números de Lê destas hipersuperfícies, incluindo o número de Milnor da hipersuperfície $X_{0}$ com singularidade isolada. Estas relações são chamadas fórmulas de Lê-Iomdine e a determinação destas é outro objetivo principal.

No capítulo 1 descrevemos os conceitos algébricos e de Teoria de Singularidade necessários para o entendimento dos números de Lê. Como os números de Lê estão associados à fibração de Milnor da hipersuperfície, no segundo capítulo é dada uma breve descrição do Teorema da 
Fibração de Milnor e suas consequências. Além disso é definido o número de Milnor para hipersuperfícies com singularidade isolada.

No capítulo 3 são definidos os números de Lê e descritas todas as propriedades básicas relacionadas a estes.

Finalmente, no capítulo 4, é mostrado como se obter recursivamente as $s$ hipersuperfícies associadas à hipersuperfície original e as fórmulas de Lê e Iomdine. 


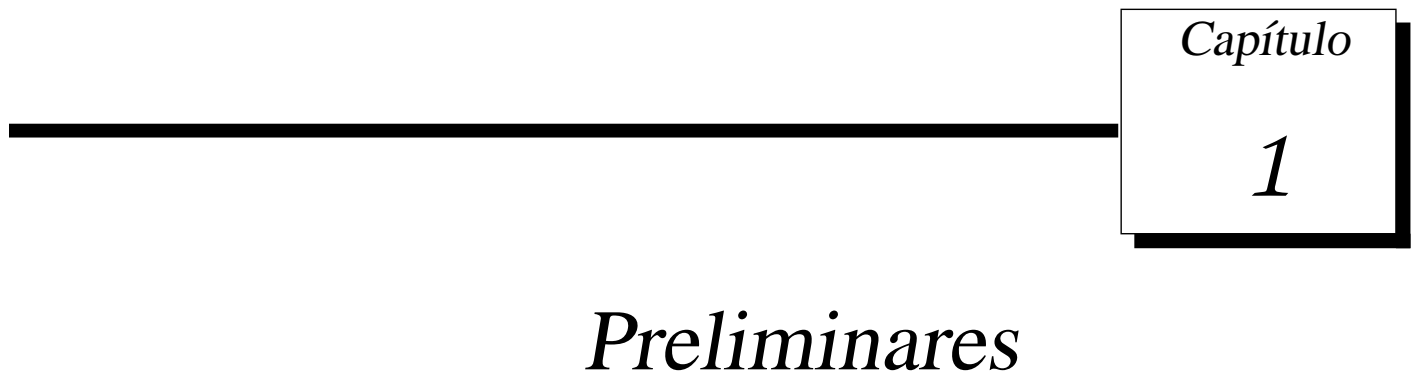

Neste capítulo damos noções básicas necessárias para o entendimento dos conceitos que são usados ao longo desse trabalho.

As referências básicas deste capítulo são Gibson [7] para os conceitos de Teoria de Singularidades e Atiyah [1], Fulton [4] e Eisenbud [3] para os conceitos de Álgebra Comutativa.

\subsection{Germes}

Sejam $X \subset \mathbb{C}^{r}$ e $Y \subset \mathbb{C}^{s}$ subconjuntos. Uma aplicação $f: X \rightarrow Y$ é chamada suave se para todo $x \in X$ existem uma vizinhança aberta $U$ de $x$ em $\mathbb{C}^{r}$ e uma aplicação holomorfa $F: U \rightarrow \mathbb{C}^{s}$ tais que $f=F$ em $U \cap X$. Dizemos que $f: X \rightarrow Y$ é um difeomorfismo se $f$ é bijetora e $f$ e $f^{-1}$ são suaves.

Definição 1.1. Uma parametrização $n$-dimensional de um conjunto $X \subset \mathbb{C}^{k}$ é uma aplicação suave $\varphi: V \rightarrow \mathbb{C}^{k}$, onde $V$ é um aberto de $\mathbb{C}^{n}$ tal que $\varphi(V)=X$ e a aplicação $\varphi: V \rightarrow \varphi(V)$ é um difeomorfismo.

Definição 1.2. Um subconjunto $N \subset \mathbb{C}^{k}$ é uma variedade diferenciável $n$-dimensional se para cada $x \in N$ existe um aberto $U$ em $\mathbb{C}^{k}$ tal que $U \cap N$ admite uma parametrização n-dimensional $\varphi: V \rightarrow \mathbb{C}^{k}$. Notação: $N^{n}$.

Definição 1.3. O espaço tangente a uma variedade $N^{n} \subset \mathbb{C}^{k}$ em um ponto $x \in N$ é a imagem da diferencial $D_{u} \varphi: \mathbb{C}^{n} \rightarrow \mathbb{C}^{k}$, onde $\varphi: V \rightarrow \mathbb{C}^{k}$ é uma parametrização de uma vizinhança de $x$ em $N^{n} \operatorname{com} \varphi(u)=x$. Notação: $T_{x} N$. 
Sejam $M^{m} \subset \mathbb{C}^{r}$ e $N^{n} \subset \mathbb{C}^{s}$ variedades, $f: M \rightarrow N$ suave, $x \in M$ e $y=f(x) \in N$. Logo existem uma vizinhança aberta $U$ de $x$ em $\mathbb{C}^{r}$ e uma aplicação de classe $\mathbb{C}^{\infty} F: U \rightarrow \mathbb{C}^{s}$ tais que $f=F$ em $U \cap M$. Temos que $D_{x} F: \mathbb{C}^{r} \rightarrow \mathbb{C}^{s}$ é uma transformação linear e que $D_{x} F\left(T_{x} M\right) \subseteq T_{y} N$.

Definição 1.4. Definimos a derivada de $f$ em $x$ como sendo a restrição de $D_{x} F$ a $T_{x} M$ e denota$\operatorname{mos} \operatorname{por} d_{x} f: T_{x} M \rightarrow T_{y} N$.

Sejam $N$ e $P$ variedades de dimensões $n$ e $p$ respectivamente, e $x \in N$. No conjunto das aplicações suaves definidas numa vizinhança de $x$ em $N$ e com valores em $P$ introduzimos a seguinte relação de equivalência $(\sim)$ :

Definição 1.5. Duas aplicações suaves $f_{1}: U_{1} \rightarrow P$ e $f_{2}: U_{2} \rightarrow P$ são equivalentes $\left(f_{1} \sim\right.$ $f_{2}$ ) quando existir uma vizinhança $U \subset U_{1} \cap U_{2}$ de $x$ em $N$ tal que as restrições $\left.f_{1}\right|_{U}$ e $\left.f_{2}\right|_{U}$ coincidem.

As classes de equivalência dessa relação são chamadas de germes de aplicações em $x$ e um elemento da classe de equivalência é chamado de representante do germe.

Notação : $f:(N, x) \rightarrow(P, y), f(x)=y$ (chamamos $N$ de fonte e $P$ de meta).

Para cada germe $f:(N, x) \rightarrow(P, y)$ temos a derivada $d_{x} f: T_{x} N \rightarrow T_{y} P$ que é definida como sendo a derivada em $x$ de qualquer representante. Dizemos que $f:(N, x) \rightarrow(P, y)$ é um germe de difeomorfismo se, e somente se, um de seus representantes (e portanto qualquer) é um difeomorfismo local, e portanto, segue do Teorema da Função Inversa que sua derivada é um isomorfismo.

No nosso caso especial que $N=\mathbb{C}^{n}$ e $P=\mathbb{C}^{p}$, denotamos por $\mathscr{O}_{n, p}$ o conjunto dos germes na origem das aplicações suaves $f:\left(\mathbb{C}^{n}, 0\right) \rightarrow \mathbb{C}^{p}$ em que não é necessariamente $f(0)=0$. Quando $p=1, \mathscr{O}_{n}$ denota este conjunto, que é um anel local cujo ideal maximal $\mathscr{M}_{n}=\left\{f \in \mathscr{O}_{n} / f(0)=\right.$ $0\}$. Então $\mathscr{O}_{n, p}$ é um $\mathscr{O}_{n}$-módulo livre com $p$-geradores.

Definição 1.6. Uma variedade analítica (algébrica) em um conjunto aberto $U \subset \mathbb{C}^{n}$ é um subconjunto de $U$ onde existe uma vizinhança aberta de cada ponto desse subconjunto que é o conjunto de zeros comuns de um número finito de funções holomorfas (polinomiais) definidas nessa vizinhança.

Observe que uma variedade analítica (algébrica) de $U$ é um subconjunto fechado de $U$. 
Considere os pares $\left(X_{\alpha}, U_{\alpha}\right)$, onde $U_{\alpha}$ é uma vizinhança da origem em $\mathbb{C}^{n}$ e $X_{\alpha}$ é uma variedade analítica (algébrica) de $U_{\alpha}$.

Definição 1.7. Dois pares $\left(X_{1}, U_{1}\right)$ e $\left(X_{2}, U_{2}\right)$ são equivalentes se existe uma vizinhança $W \subset$ $U_{1} \cap U_{2}$ da origem tal que $W \cap X_{1}=W \cap X_{2}$.

Isto define uma relação de equivalência. Uma classe de equivalência destes pares é chamado o germe de uma variedade analítica (algébrica) na origem em $\mathbb{C}^{n}$.

\subsection{Variedades e Ideais}

O desenvolvimento deste trabalho considera fortemente a relação entre germes de variedades analíticas definidas na origem em $\mathbb{C}^{n}$ e os ideais $\mathscr{O}_{n}$.

Dado um germe de variedade analítica $X$ definido na origem de $\mathbb{C}^{n}$, associamos a este o ideal $I(X)$ em $\mathscr{O}_{n}$ constituído dos germes $f \in \mathscr{O}_{n}$ tais que $f(x)=0, \forall x \in X$.

Por outro lado, para cada ideal $I$ de $\mathscr{O}_{n}, I=<f_{1}, \ldots, f_{r}>$ (que é finitamente gerado pois $\mathscr{O}_{n}$ é Noetheriano) existe a variedade canonicamente associada $V(I)=\left\{x \in \mathbb{C}^{n} \mid f_{1}(x)=\cdots=\right.$ $\left.f_{r}(x)=0\right\}$.

Um tipo especial de variedades que serão consideradas são as intersecções completas.

Definição 1.8. Seja $V^{k}$ uma variedade em $\mathbb{C}^{n}$ de dimensão $k . V^{k}$ é uma intersecção completa se $I(V)=<g_{1}, \ldots, g_{n-k}>$, ou seja, o número de geradores de $I(V)=\operatorname{codim}_{\mathbb{C}^{n}} V^{k}=n-k$.

Definição 1.9. Um germe $X$ de uma variedade analítica na origem em $\mathbb{C}^{n}$ é redutível se pode ser escrito como $X=X_{1} \cup X_{2}$, onde $X_{i}, i=1,2$ são também germes de subvariedade analíticas na origem em $\mathbb{C}^{n}$. Um germe que não é redutível é dito ser irredutível.

Observamos que um germe $X$ é irredutível se, e somente se, $I(X)$ é um ideal primo em $\mathscr{O}_{n}$ e qualquer germe $\mathrm{X}$ de uma variedade analítica na origem em $\mathbb{C}^{n}$ pode ser escrito unicamente como a união finita de variedades analíticas irredutíveis, $X=X_{1} \cup \cdots \cup X_{r}$. As variedades analíticas irredutíveis $X_{i}, 1 \leq i \leq r$ são chamadas componentes irredutíveis de $X$.

Definição 1.10. Dizemos que $X$ tem estrutura reduzida, ou simplesmente é reduzido, se $I(X)$ é igual ao seu radical $\sqrt{I(X)}$, onde $\sqrt{I(X)}=\left\{f \in \mathscr{O}_{n} \mid f^{k} \in I(X)\right.$ para algum inteiro $\left.k>0\right\}$. 
Definição 1.11. Seja $P$ um ideal de um anel A. Dizemos que $P$ é um ideal primário de A se

i) $P \varsubsetneqq A$ (ideal próprio);

ii) $\forall a, b \in A$ com $a b \in P$ mas $a \notin P$ então $b \in \sqrt{P}$.

Como $\mathscr{O}_{n}$ é Noetheriano, todo ideal $Q$ tem decomposição primária, ou seja, $Q=\bigcap_{i=1}^{r} Q_{i}$ onde $Q_{i}$ é um ideal primário.

Definição 1.12. Seja $Q$ um ideal de A. Suponhamos que $Q=\bigcap_{i=1}^{r} Q_{i}$ onde $Q_{i}$ é um ideal primário. Dizemos que Q tem decomposição primária minimal se

i) $\sqrt{Q_{i}}$ são ideais primos distintos de A;

ii) Para todo $j=1, \ldots, r$, temos $Q_{j} \nsupseteq \bigcap_{i=1, j \neq i}^{r} Q_{i}$.

Como $\mathscr{O}_{n}$ é Noetheriano, todo ideal $Q$ também tem decomposição primária minimal.

Teorema 1.13. (Teorema da Unicidade) Seja $Q$ decomponível e seja $Q=\bigcap_{i=1}^{r} Q_{i}$ uma decomposição primária minimal de $Q$. Os ideais primos $P_{i}=\sqrt{Q_{i}}, i=1, \ldots, r$ são independentes da decomposição.

Os primos $\left\{P_{1}, \ldots, P_{n}\right\}$ são chamados primos associados de $Q$ e denotamos por $\operatorname{Ass}(Q)=$ $\left\{P_{1}, \ldots, P_{n}\right\}$

Os elementos minimais de $\operatorname{Ass}(Q)$ são chamados ideais primos minimais e os outros são chamados ideais primos imersos.

Seja $X$ um germe de uma variedade analítica na origem em $\mathbb{C}^{n}, X=X_{1} \cup \cdots \cup X_{r}$ a sua decomposição em componentes irredutíveis.

Note que esses $X_{i}$ 's correspondem exatamente aos primos minimais de $I(X)$. De fato, se $P_{1}, \ldots, P_{r}$ são os ideais primos minimais de $I(X)$ então, a menos de ordem, $X_{i}=V\left(P_{i}\right)$. Daí, dizemos que $X_{i}$ é uma componente isolada de $X$.

As variedades associadas aos primos imersos de $I(X)$ são componentes mergulhadas de $X$.

Observação 1.14. Ressaltamos que toda variedade $V^{k}$ que é intersecção completa não tem componentes mergulhadas. (Ver [14] página 12) 


\subsection{Espaços Analíticos Complexos}

Definição 1.15. Seja X um espaço topológico. Um feixe $\mathscr{F}$ de anéis (respectivamente, módulos) sobre $X$, consiste do seguinte:

i) Para cada aberto $U$ de $X$, existe um anel (módulo) $\Gamma(U, \mathscr{F})$ chamado conjunto de seções de $\mathscr{F}$ sobre $U$.

ii) Para cada par de abertos $V \subseteq U$ de $X$, existe uma aplicação de restrição

$$
r_{U, V}: \Gamma(U, \mathscr{F}) \rightarrow \Gamma(V, \mathscr{F}), r_{U, V}(s)=s_{\left.\right|_{V}}
$$

satisfazendo:

i) $r_{U, U}=i d_{\Gamma(U, \mathscr{F})}$ para cada aberto $U$ de $X$;

ii) $r_{V, W} \circ r_{U, V}=r_{U, W}$ para cada $W \subseteq V \subseteq U$ abertos de $X$.

iii) Para cada aberto $U$ de $X$ e cada cobertura de $U$ por abertos $U=\cup_{i \in I} U_{i}$, de X tem-se :

i) para quaisquer $s_{1}, s_{2} \in \Gamma(U, \mathscr{F})$, se $s_{\left.1\right|_{U_{i}}}=\left.s_{2}\right|_{U_{i}} \forall i \in I$, então $s_{1}=s_{2}$;

ii) para cada $i \in I$, tomamos $s_{i} \in \Gamma\left(U_{i}, \mathscr{F}\right)$. Se $s_{\left.i\right|_{U_{i} \cap U_{j}}}=s_{j_{\left.\right|_{i} \cap U_{j}}}$ para todo $i, j \in I$, então existe $s \in \Gamma(U, \mathscr{F})$ tal que $s_{\left.\right|_{i}}=s_{i}$ para todo $i \in I$.

Exemplo 1.16. Em $\mathbb{C}^{n}$, considerando a topologia usual, definimos o feixe $\mathscr{O}_{\mathbb{C}^{n}}$ da seguinte maneira.

Dado $U \subseteq \mathbb{C}^{n}$ aberto, $\mathscr{O}_{\mathbb{C}^{n}}(U):=\{f: U \rightarrow \mathbb{C} \mid f$ holomorfa $\}$. Para $V \subseteq U$ abertos, consideremos as restrições naturais $\rho_{V}^{U}: \mathscr{O}_{\mathbb{C}^{n}}(U) \rightarrow \mathscr{O}_{\mathbb{C}^{n}}(V), \operatorname{com} \rho_{V}^{U}(s)=s_{\left.\right|_{V}}$.

É fácil verificar que $\mathscr{O}_{\mathbb{C}^{n}}$ é um feixe de anéis.

Definição 1.17. Seja $\mathscr{F}$ um feixe de anéis (módulos) sobre X. Definimos o talo de $\mathscr{F}$ em x como o anel (módulo)

$$
\mathscr{F}_{x}:=\{(U, s): U \text { é aberto de } X, x \in U \text { e } s \in \Gamma(U, \mathscr{F})\}
$$

módulo a relação $\sim$

$(U, s) \sim(V, t) \Leftrightarrow \exists W$ aberto de $X$ contido em $U \cap V$ tal que $s_{\left.\right|_{W}}=t_{\left.\right|_{W}}$.

Ressaltamos que $\mathscr{O}_{\mathbb{C}^{n}, 0}=\mathscr{O}_{n}$ é o anel local de germes de funções holomorfas de $\mathbb{C}^{n}$ em 0 . 
Definição 1.18. Um espaço anelado é um par $(X, \mathscr{F})$ onde $X$ é um espaço topológico e $\mathscr{F}$ é um feixe de anéis.

Exemplo 1.19. Seja $D \subseteq \mathbb{C}^{n}$ domínio com a topologia induzida. Para cada aberto $U$ de $D$ consideremos $\mathscr{O}_{D}(U):=\{f: U \rightarrow \mathbb{C} \mid f$ holomorfa $\}$. Novamente considerando as restrições naturais temos que $\mathscr{O}_{D}$ é um feixe de anéis sobre $D$.

É claro que $\mathscr{O}_{D, x} \cong \mathscr{O}_{\mathbb{C}^{n}, x}, \forall x \in D$

Definição 1.20. Um morfismo $\alpha: \mathscr{F} \rightarrow \mathscr{G}$ entre dois feixes de X é uma coleção de aplicações

$$
\alpha(V): \Gamma(V, \mathscr{F}) \rightarrow \Gamma(V, \mathscr{G})
$$

para cada $V$ aberto de $X$ tal que o diagrama

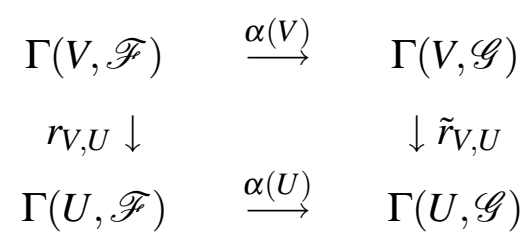

comuta sempre que $U \subset V$ são abertos de $X$.

Um morfismo $\alpha: \mathscr{F} \rightarrow \mathscr{G}$ induz um homomorfismo natural $\alpha_{x}: \mathscr{F}_{x} \rightarrow \mathscr{G}_{x}$ para todo $x \in X$. O morfismo $\alpha$ é injetor (sobrejetor) se para todo $x \in X, \alpha_{x}$ é injetor (sobrejetor).

Definição 1.21. Consideremos o espaço anelado $(X, \mathscr{F})$. Seja $Z$ um subespaço topológico de $X$. Definimos $\mathscr{F}_{\mid Z}$ como sendo o feixe onde $\Gamma\left(V, \mathscr{F}_{\mid Z}\right):=\lim _{U \supseteq i(V)} \Gamma(U, \mathscr{F})$ (limite direto), onde $V$ é um aberto de $Z, U$ é um aberto de $X$ e i é a aplicação inclusão.

Definição 1.22. Seja $\psi: X \rightarrow Y$ contínua e $\mathscr{F}$ um feixe sobre $X$. Para cada aberto $U$ de $Y$ $\operatorname{definimos}\left(\psi_{*}(\mathscr{F})\right)(U)=\mathscr{F}\left(\psi^{-1}(U)\right)$.

Verifica-se que $\psi_{*}(\mathscr{F})$ é um feixe sobre $Y$.

Definição 1.23. Um espaço analítico complexo é um espaço anelado $\left(X, \mathscr{O}_{X}\right)$ onde $\mathscr{O}_{X}$ é um feixe de $\mathbb{C}$ álgebra locais (isto é, $\mathscr{O}_{X, x}$ é uma $\mathbb{C}$-álgebra local, $\forall x \in X$ ) e tal que para cada aberto $U \subseteq X,\left(U, \mathscr{O}_{\left.X\right|_{U}}\right)$ é isomorfo a $\left(D, \mathscr{O}_{D}\right)$ para algum domínio $D \subseteq \mathbb{C}^{n}$, isto é, existe um $\operatorname{par}(\varphi, \widetilde{\varphi})$ tal que $\varphi: U \rightarrow D$ é homeomorfismo e $\widetilde{\varphi}: \mathscr{O}_{D} \rightarrow \varphi_{*}\left(\mathscr{O}_{\left.X\right|_{U}}\right)$ é isomorfismo de feixes. 
No decorrer no texto, para simplificar a notação, denotamos por $\mathscr{O}_{U}$ o feixe $\mathscr{O}_{\left.X\right|_{U}}$, onde $U$ é um aberto de $X$.

Observação 1.24. $\left(\mathbb{C}^{n}, \mathscr{O}_{\mathbb{C}^{n}}\right)$ é um espaço analítico complexo.

Definição 1.25. Sejam $\left(X, \mathscr{O}_{X}\right)$ e $\left(Y, \mathscr{O}_{Y}\right)$ espaços analíticos complexos. Um morfismo entre eles é um par $(\varphi, \widetilde{\varphi})$ tal que $\varphi: Y \rightarrow X$ é contínua e $\widetilde{\varphi}: \mathscr{O}_{X} \rightarrow \varphi_{*}\left(\mathscr{O}_{Y}\right)$ é morfismo de feixes.

Notação: $(\varphi, \widetilde{\varphi}):\left(Y, \mathscr{O}_{Y}\right) \rightarrow\left(X, \mathscr{O}_{X}\right)$.

Definição 1.26. Sejam $\left(X, \mathscr{O}_{X}\right)$ e $\left(Y, \mathscr{O}_{Y}\right)$ espaços analíticos complexos. Uma imersão fechada é um morfismo $(\varphi, \widetilde{\varphi}):\left(Y, \mathscr{O}_{Y}\right) \rightarrow\left(X, \mathscr{O}_{X}\right)$ tal que $\varphi(Y) \subseteq X$ é fechado e $\widetilde{\varphi}$ é sobrejetora.

A esta imersão fechada associamos um "feixe" $\mathscr{I}_{Y}$ sobre $X$ da seguinte maneira:

$\mathscr{I}_{Y}:=\operatorname{Ker}(\widetilde{\varphi})$, isto é, $\mathscr{I}_{Y}(U):=\operatorname{Ker}(\widetilde{\varphi}(U))$, onde $\widetilde{\varphi}(U): \mathscr{O}_{X}(U) \rightarrow \varphi_{*}\left(\mathscr{O}_{Y}\right)(U)$.

Temos que $\mathscr{I}_{Y}(U)$ é um ideal de $\mathscr{O}_{X}(U), \forall U \subseteq X$ aberto. Dizemos, neste caso, que $\mathscr{I}_{Y}$ é um feixe de ideais.

Além disso, se $\mathscr{O}_{X}$ é um feixe de anéis Noetherianos então $\mathscr{I}_{Y}(U)$ é finitamente gerado, para todo aberto $U$ de $X$. Neste caso, dizemos que $\mathscr{I}_{Y}$ é um feixe coerente de ideais.

Durante todo o trabalho denotaremos o espaço analítico complexo $\left(X, \mathscr{O}_{X}\right)$ simplesmente por $X$, ficando subentendido seu feixe. Como componentes irredutíveis do espaço analítico complexo $X$ queremos dizer as componentes irredutíveis do espaço topológico $X$.

\subsection{Ciclos Analíticos}

Seja $S$ um conjunto não vazio. Consideremos o conjunto $\mathbb{Z}(S)=\{f: S \rightarrow \mathbb{Z} \mid f(s)=0$ para quase todo $s \in S$ exceto para um número finito $\}$.

Com a adição usual de aplicações temos que $\mathbb{Z}(S)$ é um grupo abeliano.

Dado $f \in \mathbb{Z}(S)$ existem finitos $s_{1}, \ldots, s_{n} \in S$ tais que $f\left(s_{i}\right) \neq 0$. Denotemos $f(s)=a_{s}, \forall s \in S$. Assim cada elemento $f \in \mathbb{Z}(S)$ pode ser escrito na forma simbólica $\sum_{s \in S} a_{s}[s]$.

Notação: $f=\sum_{s \in S} a_{s}[s]=\sum_{i} a_{s_{i}}\left[s_{i}\right]$.

Para $f=\sum_{s \in S} a_{S}[s]$ e $g=\sum_{s \in S} b_{s}[s]$ temos $f+g=\sum_{s \in S} a_{s}[s]+\sum_{s \in S} b_{S}[s]=\sum_{s \in S}\left(a_{s}+b_{s}\right)[s]$.

Uma base para o grupo $\mathbb{Z}(S)$ é o conjunto das funções características $\chi_{s}: S \rightarrow \mathbb{Z}$ com 


$$
\chi_{s}(t)=\left\{\begin{array}{lll}
0 & \text { se } & t \neq s \\
1 & \text { se } & t=s
\end{array}, \text { isto é, } \chi_{s}=1[s]\right.
$$

Logo $\mathbb{Z}(S)$ é um grupo abeliano livre chamado de grupo abeliano livre gerado por $S$.

Seja $X$ um espaço analítico complexo de dimensão $d$. Dado $0 \leq k \leq d$ definimos $S_{k}:=\{V \mid V$ é um subespaço analítico complexo irredutível de $X$ e $\operatorname{dim} V=k\}$.

Denotamos $\mathbb{Z}\left(S_{k}\right)$ por $\mathbb{Z}_{k}(X)$.

Um $k$-ciclo é um elemento deste grupo abeliano livre gerado por $S_{k}$, isto é, é um elemento da forma $\sum_{V \in S_{k}} \eta_{V}[V] \in \mathbb{Z}_{k}(X)$.

Define-se $\mathbb{Z}(X):=\oplus_{k=0}^{d} \mathbb{Z}_{k}(X)$. Os elementos de $\mathbb{Z}(X)$ são chamados de ciclos de $X$.

Consideremos agora um espaço analítico complexo $X$ com estrutura reduzida. Sejam $V_{1}, \ldots, V_{r}$ as suas componentes irredutíveis. O ciclo analítico associado a $X$, denotado por $[X]$, é o elemento $\sum_{i=1}^{r} \eta_{V_{i}}\left[V_{i}\right]$ de $\mathbb{Z}(X)$, onde os $\eta_{V_{i}}$ são definidos como segue.

Tome um ponto $p$ em $V_{i}$ e considere o germe de $V_{i}$ em $p$. Sejam $\left(V_{i p}\right)_{j}$ as componentes irredutíveis deste germe.

Tome algum dos $\left(V_{i p}\right)_{j}$ e seja $\eta_{V_{i}}$ igual ao comprimento do anel $\left(\mathscr{O}_{V_{i}, p}\right)_{\left(\widetilde{V_{i p}}\right)_{j}}$, onde $\left(\widetilde{V_{i p}}\right)_{j}$ é o ideal primo correspondente a $\left(V_{i p}\right) j$.

Massey observa na página 9 de [14] que esse número independe do ponto $p$ e da escolha de $\left(V_{i p}\right)_{j}$.

Com essa notação, é fundamental que, se $f, g \in \mathscr{O}_{U}$ então $[V(f g)]=[V(f)]+[V(g)]$ e, em particular, $\left[V\left(f^{m}\right)\right]=m[V(f)]$.

Observação 1.27. Sejam $V$ e $W$ subespaços analíticos complexos de $X$. Se os ciclos $[V]$ e $[W]$ são iguais, então como espaços analíticos complexos, $V$ e $W$ são iguais a menos de componentes mergulhadas.

Durante o trabalho denotamos $[X]$ para o ciclo e o conjunto analítico com um par de linhas verticais $|X|$. Ocasionalmente, a notação será simplificada, explicitando se estamos considerando $X$ como um espaço analítico complexo, um ciclo ou um conjunto. 


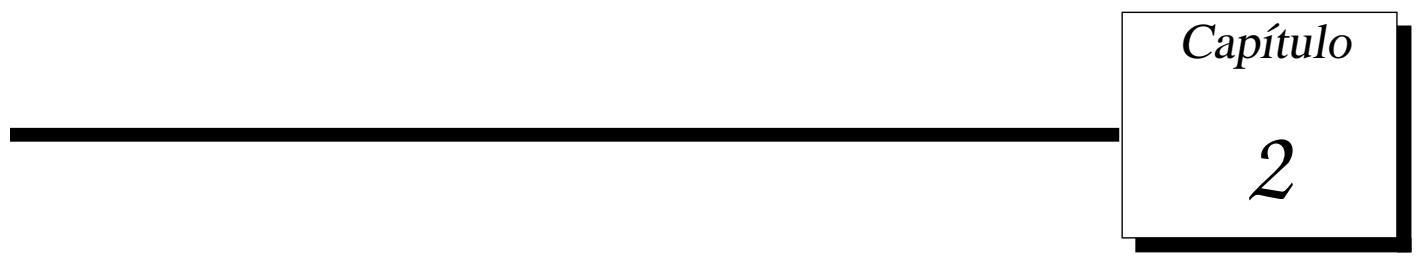

\section{O teorema da fibração e o número de Milnor}

\subsection{Teorema da fibração}

Nesta secção descrevemos os principais resultados de Milnor em [16].

Sejam $f:\left(\mathbb{C}^{n+1}, 0\right) \rightarrow(\mathbb{C}, 0)$ um germe de função não nula em $\mathscr{O}_{n}$ e $V=f^{-1}(0)$ a hipersuperfície complexa definida por $f$.

Dado $\varepsilon>0$, consideremos $D_{\varepsilon}=\left\{z \in \mathbb{C}^{n+1}:\left|z-z^{o}\right| \leq \varepsilon\right\}, S_{\varepsilon}=\left\{z \in \mathbb{C}^{n+1}:\left|z-z^{o}\right|=\varepsilon\right\} \mathrm{e}$ $K=V \cap S_{\varepsilon}$.

O teorema a seguir estabelece uma relação entre a topologia de $K$ e a de $V$.

Teorema 2.1. Para $\varepsilon>0$ suficientemente pequeno $V \cap D_{\varepsilon}$ é homeomorfo ao cone $C(K)=\{t z$ : $0 \leq t \leq 1, z \in K\}$

Seja $\phi: S_{\varepsilon}-K \rightarrow S^{1}$ dada por $\phi(z)=\frac{f(z)}{|f(z)|}, \operatorname{com} \phi^{-1}\left(e^{i \theta}\right)=F_{\theta}$.

O resultado básico da teoria de Milnor é o teorema da fibração, que mostra que $\phi$ define uma fibração de $S_{\varepsilon}-K$ sobre $S^{1}$ com fibra $F_{\theta}$.

Teorema 2.2. Se $z^{o}$ é um ponto qualquer de $V$ e se $S_{\varepsilon}$ é uma esfera suficientemente pequena centrada em $z^{o}$ então $S_{\varepsilon}-K$ é um fibrado diferenciável localmente trivial sobre $S^{1}$, com projeção $\phi(z)=\frac{f(z)}{|f(z)|}$ e fibra $F_{\theta}=\phi^{-1}\left(e^{i \theta}\right)$.

Corolário 2.3. Para $\varepsilon>0$ suficientemente pequeno, a aplicação $\phi: S_{\mathcal{\varepsilon}}-K \rightarrow S^{1}$ não tem pontos críticos. 
Segue que, para cada $e^{i \theta} \in S^{1}$, a imagem inversa $F_{\theta}=\phi^{-1}\left(e^{i \theta}\right) \subset S_{\varepsilon}-K$ é uma variedade diferenciável de dimensão $2 n$.

Teorema 2.4. Cada fibra $F_{\theta} \subset S_{\varepsilon}-K$ tem o mesmo tipo de homotopia de um complexo $C W$ finito n-dimensional.

Isso implica que todos os grupos de Homologia são finitamente gerados, são zero acima do grau n, e que $H_{n}\left(F_{\theta}\right)$ é abeliano livre.

Teorema 2.5. A fibra $F_{\theta} e ́(n-1)$-conexa.

Teorema 2.6. Se o número complexo $c \neq 0$ tem módulo suficientemente pequeno, então a hipersuperfície complexa $f^{-1}(c)$ intercepta o disco $\stackrel{\circ}{D_{\varepsilon}}$ numa variedade diferenciável difeomorfa a fibra $F_{\theta}$.

O teorema anterior nos leva a considerar uma fibração equivalente, porém mais conveniente que vive dentro da bola de raio $\varepsilon$. Daí, durante esse trabalho, é usada esta fibração de Milnor como descrita abaixo.

Para todo $\varepsilon>0$, seja $\stackrel{\circ}{B_{\varepsilon}}$ a bola aberta de raio $\varepsilon$ centrada na origem em $\mathbb{C}^{n+1}$. Para todo $\eta>0$, sejam $\mathbb{D}_{\eta}$ o disco fechado centrado na origem em $\mathbb{C}$ e $\partial \mathbb{D}_{\eta}$ denotando sua fronteira, que é um círculo de raio $\eta$. Então, tendo fixado uma função analítica, $f$, existe $\varepsilon_{0}>0$ tal que, para todo $\varepsilon$ tal que $0<\varepsilon \leq \varepsilon_{0}$, exista $\eta_{\varepsilon}>0$ tal que, para todo $\eta$ tal que $0<\eta \leq \eta_{\varepsilon}$, a restrição de $f$ por uma aplicação $\stackrel{\circ}{B_{\varepsilon}} \cap f^{-1}\left(\partial \mathbb{D}_{\eta}\right) \rightarrow \partial \mathbb{D}_{\eta}$ é uma fibração localmente trivial suave cujo tipo de difeomorfismo independe da escolha de $\varepsilon$ e $\eta$.

Essa fibração é chamada de Fibração de Milnor de $f$ na origem e a fibra é a fibra de Milnor de $f$ na origem, que a partir daqui é denotada por $F_{f, 0}$.

Para fazer um exemplo, existe um resultado final de Milnor que devemos mencionar aqui. Suponha que $f$ é um polinômio quase homogêneo, isto é, existem inteiros positivos $r_{0}, \ldots, r_{n}$ tais que $f\left(z_{0}^{r_{0}}, \ldots, z_{n}^{r_{n}}\right)$ é um polinômio homogêneo (Neste caso, dizemos que $f$ é quase homogêneo do tipo $\left(r_{0}, \ldots, r_{n} ; d\right)$, onde $d$ é o grau de homogeneidade de $\left.f\right)$. Então, ([16], Lema 9.4) a fibra de Milnor, $F_{f, 0}$, é difeomorfa a $f^{-1}(1)$. 
Exemplo 2.7. Considere $f=x y z$, que define uma hipersuperfície em $\mathbb{C}^{3}$ consistindo dos três planos coordenados. Assim, $V(f)$ é uma hipersuperfície com um conjunto singular 1dimensional consistindo dos três eixos coordenados.

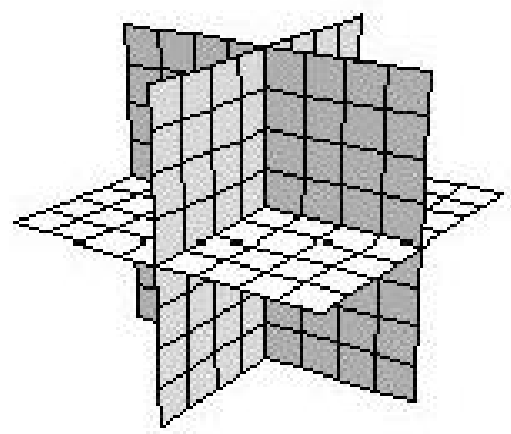

Figura 2.1: Os hiperplanos coordenados

Pelo resultado de polinômios quase homogêneos, a fibra de Milnor é difeomorfa ao conjunto de pontos onde $x y z=1$; mas, isso é onde $y \neq 0, z \neq 0$ e $x=\frac{1}{y z}$.

Assim $F_{f, 0} \cong \mathbb{C}^{*} \times \mathbb{C}^{*}$, onde $\mathbb{C}^{*}=\mathbb{C}-0$, pois $y \neq 0$ e $z \neq 0$, então $F_{f, 0}$ é homotopicamente equivalente ao produto de dois círculos, e deste modo tem homologia não nula nos graus $0,1 \mathrm{e}$ 2.

Exemplo 2.8. O guarda-chuva de Whitney é a hipersuperfície em $\mathbb{C}^{3}$ definida pelo anulamento de $f=y^{2}-z x^{2}$.

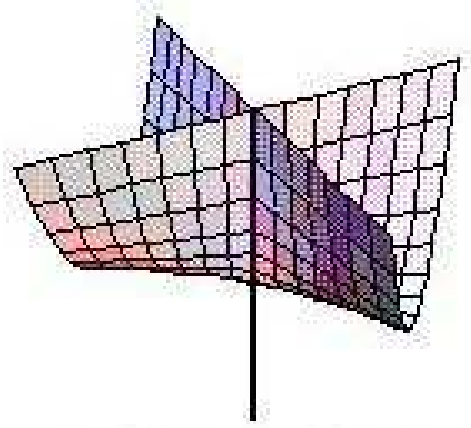

Figura 2.2: O guarda-chuva de Whitney 
Aqui, a figura é desenhada considerando os números reais - este é o quadro raramente visto que explica a palavra "guarda-chuva" no nome deste exemplo. No caso complexo, a dimensão de todas as componentes de $X$ é sempre constante, portanto não aparece o "cabo do guardachuva". Uma segunda razão por que raramente se vê a figura anterior é que frequentemente o guarda-chuva de Whitney é encontrado como uma família de nós que se degeneram em uma cúspide; esta representação é alcançada fazendo a mudança analítica de coordenadas $z=x+t$ para obter $f=y^{2}-x^{3}-t x^{2}$.

Para determinar o tipo de homotopia da fibra de Milnor do guarda-chuva de Whitney na origem, é necessário um novo resultado:

Teorema 2.9. Se $f:(U, 0) \rightarrow(\mathbb{C}, 0)$ e $g:\left(U^{\prime}, 0\right) \rightarrow(\mathbb{C}, 0)$ são funções analíticas, então a fibra de Milnor da função $h:\left(U \times U^{\prime}, 0\right) \rightarrow(\mathbb{C}, 0)$ definida por $h(w, z):=f(w)+g(z)$ é homotopicamente equivalente a uma junção, $F_{f, 0} * F_{g, 0}$ das fibras de Milnor de $f$ e $g$.

A demonstração deste resultado pode ser vista em várias formas em [18], [19] e [20].

Isso determina a homologia de $F_{h, 0}$ em um caminho simples, desde que a homologia reduzida da junção de dois espaços $X$ e $Y$ seja dada por

$$
\widetilde{H}_{j+1}(X * Y)=\sum_{k+l=j} \widetilde{H}_{k}(X) \otimes \widetilde{H}_{l}(Y) \oplus \sum_{k+l=j-1} \operatorname{Tor}\left(\widetilde{H}_{k}(X), \widetilde{H}_{l}(Y)\right) .
$$

Em especial, dada uma função analítica $g\left(z_{0}, \ldots, z_{n}\right)$ e uma variável $y$, disjunta das $z^{\prime} s$, então a fibra de Milnor de $y^{2}+g\left(z_{0}, \ldots, z_{n}\right)$ é homotopicamente equivalente a suspensão da fibra de Milnor de $g$. Por um abuso de linguagem, frequentemente dizemos que a singularidade de $y^{2}+g\left(z_{0}, \ldots, z_{n}\right)$ é a suspensão da singularidade de $g$.

Assim, no exemplo, $F_{y^{2}-z x^{2}, 0}$ é homotopicamente equivalente a suspensão de $F_{z x^{2}, 0}$. Mas, como $z x^{2}$ é homogênea,

$$
F_{z x^{2}, 0} \cong\left\{(z, x) \mid z x^{2}=1\right\}=\left\{\left(\frac{1}{x^{2}}, x\right) \mid x \neq 0\right\} \cong \mathbb{C}^{*}
$$

Assim, $F_{y^{2}-z x^{2}, 0}$ é homotopicamente equivalente a suspensão de um círculo, isto é, a fibra de Milnor de $f$ na origem é homotopicamente equivalente a uma 2-esfera. 


\subsection{Teorema da fibração de Lê}

O resultado de Lê em [12] descrito a seguir permite calcular a homologia da fibra de Milnor para uma hipersuperfície singular arbitrária.

Sejam $U$ uma vizinhança aberta da origem em $\mathbb{C}^{n+1}, f:(U, 0) \rightarrow(\mathbb{C}, 0)$ uma função analítica e $L:\left(\mathbb{C}^{n+1}, 0\right) \rightarrow(\mathbb{C}, 0)$ uma função linear genérica.

Teorema 2.10. A fibra de Milnor $F_{f, 0}$ é obtida da fibra de Milnor $F_{f_{V_{V(L)}}, 0}$ pela união de um certo número de n-alças (n-célula no nível de homotopia); esse número de n-alças unidas é dado pelo número de intersecções $\left(\Gamma_{f, L}^{1} \cdot V(f)\right)_{0}$, onde L é uma aplicação linear genérica e $\Gamma_{f, L}^{1}$ denota a curva polar relativa de $f$ com respeito a $L$.

Observação 2.11. Os números de n-alças dão origem aos números de Lê.

No capítulo 3 é definida a curva polar e discutido como calcular números de intersecções.

O resultado de Lê ganha importância pois permite calcular algumas informações importantes até mesmo nos casos onde o tipo de homotopia da fibra de Milnor não pode ser determinada por outros meios.

\subsection{O número de Milnor}

Nesta secção descrevemos os resultados de Milnor em [16] e [2].

Quando $f$ tem singularidade isolada em 0 , o teorema abaixo caracteriza o tipo de homotopia da fibra $F_{f, 0}$.

Teorema 2.12. Cada fibra $F_{f, 0}$ tem o mesmo tipo de homotopia de um bouquet $S^{n} \vee \ldots \vee S^{n}$ de esferas.

Definição 2.13. O número de esferas $S^{n}$ do bouquet, ou o número de geradores da homologia média da fibra $F_{f, 0}$ é chamado número de Milnor de $f$ em 0 e é denotado por $\mu(f)$.

Mais precisamente temos que

$$
H_{j}\left(F_{f, 0}\right)=\left\{\begin{array}{cccc}
\mathbb{Z} & \text { se } & j=0 \\
0 & \text { se } & j \neq 0 \text { e } j \neq n \\
\mathbb{Z} \oplus \cdots \oplus \mathbb{Z} & \text { se } & j=n
\end{array}\right.
$$


onde $\mu(f)=$ posto $H_{n}\left(F_{f, 0}\right)$.

Definição 2.14. Duas funções holomorfas $f$ e $g:\left(\mathbb{C}^{n+1}, 0\right) \rightarrow(\mathbb{C}, 0)$ com 0 como ponto crítico isolado de $f$ e g possuem o mesmo tipo topológico local em 0 se existem vizinhanças $U$ e $V$ de 0 em $\mathbb{C}^{n+1}$ e um homeomorfismo $\psi:(U, 0) \rightarrow(V, 0)$ tais que $\psi\left(f^{-1}(0) \cap U\right)=g^{-1}(0) \cap V$.

O seguinte teorema mostra que o número de Milnor é um invariante completo do tipo topológico de V.

Teorema 2.15. (Lê Dûng Tráng) Se dois germes $f$ e g possuem o mesmo tipo topológico então $\mu(f)=\mu(g)$.

Definição 2.16. A multiplicidade algébrica de $f$, denotada por $\mu_{a}(f)$, é dada por $\mu_{a}(f)=\operatorname{dim}_{\mathbb{C}} \frac{\mathscr{O}_{n+1}}{J(f)}$, onde $J(f)$ denota o ideal jacobiano $\left\langle\frac{\partial f}{\partial z_{0}}, \ldots, \frac{\partial f}{\partial z_{n}}\right\rangle$.

Teorema 2.17. $\mu_{a}(f)=\mu(f)$.

No caso de polinômios quase homogêneos, Milnor e Orlik em [17] mostram como calcular o número de Milnor.

Teorema 2.18. Seja $f\left(z_{0}, \ldots, z_{n}\right)$ um polinômio quase-homogêneo do tipo $\left(r_{0}, \ldots, r_{n} ; d\right)$ com um ponto crítico isolado na origem. Então

$$
\mu=\frac{\left(d-r_{0}\right)\left(d-r_{1}\right) \cdots\left(d-r_{n}\right)}{r_{0} \ldots r_{n}}
$$

Retornando agora ao exemplo 2.7 onde $f=x y z$, foi visto que $F_{f, 0}$ não precisa ter o mesmo tipo de homotopia de um bouquet de esferas se a singularidade não é isolada. Contudo, existe um resultado mais geral de Kato e Matsumoto [9]:

Teorema 2.19. Se $s:=\operatorname{dim}_{0} \Sigma(f)$, então $F_{f, 0} e ́(n-s-1)$ - conexo; em particular, quando $s=0$, recuperamos o resultado de Milnor.

Assumindo que $\left(\Gamma_{f, L}^{1} \cdot V(f)\right)_{0}$ é efetivamente calculável, é possível ver que os resultados de Kato e Matsumoto seguem intuitivamente do Teorema 2.10 e do fato que unindo alças de índice $\mathrm{k}$ não afeta a conexão em dimensões $\leq k-2$. 


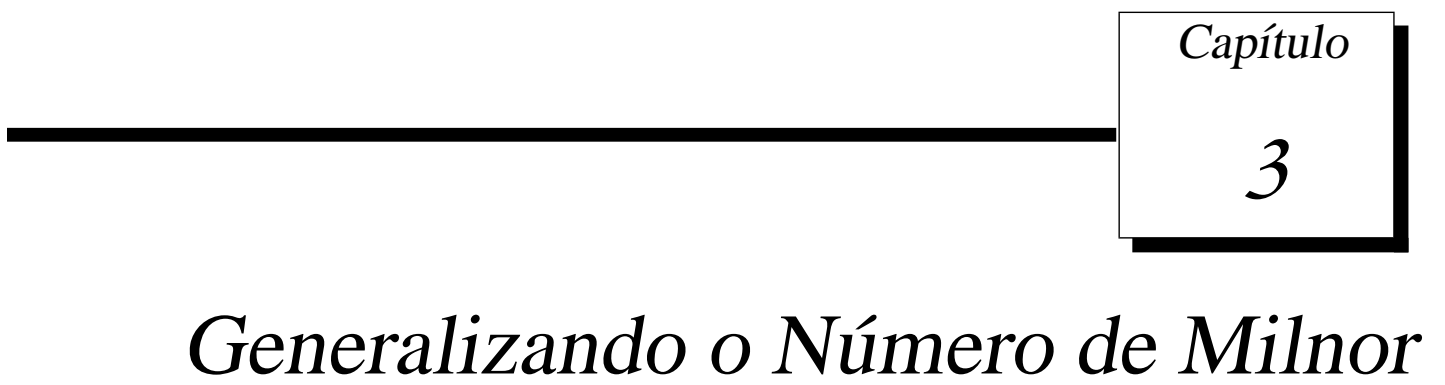

Neste capítulo, são definidos e demonstrados resultados sobre os objetos fundamentais do estudo deste trabalho - os ciclos de Lê e os números de Lê.

Durante todo esse capítulo, $U$ denotará um subconjunto aberto de $\mathbb{C}^{n+1}$ contendo a origem e $h:(U, 0) \rightarrow(\mathbb{C}, 0)$ será uma função analítica tal que $\operatorname{dim}_{0} \Sigma(h)=s$.

Então a seguinte questão se coloca naturalmente: Quais propriedades devemos ter para generalizar o número de Milnor na origem?

Primeiro, associado a $h$, queremos encontrar $s+1$ números que são efetivamente calculáveis; chamemos os números $\lambda_{h}^{0}, \ldots, \lambda_{h}^{s}$. No caso de singularidade isolada, queremos que $\lambda_{h}^{0}$ seja o número de Milnor de $h$ e qualquer outro $\lambda_{h}^{i}$ seja zero.

Para $s$ arbitrário, gostaríamos de generalizar o resultado de Milnor para singularidades isoladas e mostrar que a fibra de Milnor de $h$ na origem tem uma decomposição em alças na qual o número de alças de cada índice é obtido como $\lambda_{h}^{i}$. Os números de Lê se mostram como os números adequados para este objetivo.

\subsection{Ciclos polares}

Veremos que os ciclos de Lê são ciclos analíticos que decompõem o lugar crítico de uma função analítica e que os números de Lê são números de intersecções dos ciclos de Lê com subespaços lineares genéricos. Observemos que o termo genérico aqui é usado para descrever os planos que possuem o maior número possível de intersecções com os ciclos. 
Para definir os ciclos de Lê, primeiro necessitamos definir ciclos polares, que são ciclos associados a variedades polares relativas. Por isso, começamos por discutir as operações algébricas que são usadas para obter uma variedade polar relativa não reduzida.

Um espaço $X$ é dito ser puramente $k$-dimensional no ponto $p$, se a dimensão em $p$ de qualquer uma de suas componentes é $k$ ou $X$ não tem nenhuma componente por $p$.

Sejam $V$ e $W$ dois espaços analíticos complexos irredutíveis em um subconjunto aberto $U$ de algum espaço afim. Dizemos que $V$ e $W$ se interceptam propriamente em $U$ desde que codim $V \cap W=\operatorname{codim} V+\operatorname{codim} W$. Então, neste caso, a intersecção produto de $[V]$ e $[W]$ é definida por $[V] \cdot[W]=[V \cap W]$.

Dois ciclos $\sum m_{i}\left[V_{i}\right]$ e $\sum n_{j}\left[V_{j}\right]$ são ditos que se interceptam propriamente se $V_{i}$ e $W_{j}$ se interceptam propriamente, $\forall i, j$. Então neste caso a intersecção produto é estendida bilinearmente pela definição

$$
\sum m_{i}\left[V_{i}\right] \cdot \sum n_{j}\left[W_{j}\right]=\sum m_{i} n_{j}\left(\left[V_{i}\right] \cdot\left[W_{j}\right]\right)=\sum m_{i} n_{j}\left(\left[V_{i} \cap W_{j}\right]\right)
$$

Se dois ciclos $C_{1}$ e $C_{2}$ se interceptam propriamente e $C_{1} \cdot C_{2}=\sum p_{k}\left[Z_{k}\right]$ onde $Z_{k}$ são irredutíveis então o número de intersecções de $C_{1}$ e $C_{2}$ em $Z_{k},\left(C_{1} \cdot C_{2}\right) Z_{k}$, é definido como sendo $p_{k}$; que é o número de vezes que $Z_{k}$ acontece na intersecção, contando com multiplicidade. Durante todo o trabalho, usamos números de intersecções somente quando $C_{1}$ e $C_{2}$ têm codimensões complementares; neste caso, todos os $Z_{k}$ são simplesmente pontos.

Finalmente, dados um ponto $p \in U$, uma curva $W$ em $U$ que é irredutível em $p$ e uma hipersuperfície $V(f) \subseteq U$ que intercepta $W$ propriamente em $p$, existe um modo muito útil para calcular o número de intersecções $([W] \cdot[V(f)])_{p}$. Toma-se uma parametrização local $\phi(t)$ de $W$ que leva 0 em $p$, e então $([W] \cdot[V(f)])_{p}=$ mult $_{t} f(\phi(t))=$ o grau do menor termo não nulo.

Exemplo 3.1. Seja $U$ um aberto em $\mathbb{C}^{2}$ contendo a origem. Seja $W$ a reta $y=x$ em $U$. Considere como parametrização local de $W$ a aplicação $\phi(t)=(t, t)$. Seja $f=x^{2}-y^{3}$. Temos que $V(f) \subset U$ intercepta $W$ propriamente na origem pois $\operatorname{codim} V(f)=1, \operatorname{codim} W=1$ e $\operatorname{codim}(V(f) \cap W)=$ 
2 (a intersecção é um ponto). Portanto

$$
([W] \cdot[V(f)])_{0}=\text { mult }_{t} f(\phi(t))=\text { mult }_{t}\left(t^{2}-t^{3}\right)=2 .
$$

Agora, desejamos definir o artifício algébrico que usaremos para definir variedades polares relativas como possíveis espaços analíticos complexos não redutíveis.

Seja $W$ um subconjunto analítico de algum subconjunto aberto $U$ em algum espaço afim e seja $\alpha$ um feixe coerente de ideais em $\mathscr{O}_{U}$.

Em cada ponto $x \in V(\alpha)$ desejamos considerar as componentes do espaço analítico complexo $V(\alpha)$ passando por $x$ que não estão contidas em $|W|$.

Definição 3.2. Consideremos $\alpha_{x}$ o talo de $\alpha$ em $\mathscr{O}_{U, x}$. Seja $S$ o conjunto multiplicativamente fechado $\mathscr{O}_{U, x}-\bigcup p$, onde a união é para todo $p \in A s s\left(\mathscr{O}_{U, x} / \alpha_{x}\right)$ com $|V(p)| \nsubseteq|W|$. Então, definimos $\alpha_{x} / W$ igual a $S^{-1} \alpha_{x} \cap \mathscr{O}_{U, x}$. Assim, $\alpha_{x} / W$ é o ideal em $\mathscr{O}_{U, x}$ consistindo da intersecção destas componentes primárias, $q$ (possivelmente mergulhadas), de $\alpha_{x}$ tais que $|V(q)| \nsubseteq|W|$.

Agora, tendo definido $\alpha_{x} / W$ em cada talo, por [21], se executamos essa operação simultaneamente em todos os pontos de $V(\alpha)$, então obtemos um feixe coerente de ideais chamado feixe lacuna; escrevemos esse feixe como $\alpha / W$. Se $V=V(\alpha), V / W$ é denotado pelo espaço analítico complexo $V(\alpha / W)$.

Massey observa em [14] que $V / W$ não depende da estrutura de $W$ como um espaço analítico complexo mas somente como um conjunto analítico.

Exemplo 3.3. Seja $f=y^{2}-x^{3}-t x^{2}$. Considere

$$
W=V\left(\frac{\partial f}{\partial t}, \frac{\partial f}{\partial x}, \frac{\partial f}{\partial y}\right)=V\left(-x^{2},-3 x^{2}-2 t x, 2 y\right)=V(x, y)
$$

Seja $\alpha=\left\langle\frac{\partial f}{\partial x}, \frac{\partial f}{\partial y}\right\rangle=<-3 x^{2}-2 t x, 2 y>$. 
Se $t \neq 0$, as componentes de $V(\alpha)$ são: $V_{1}:=\left\{\begin{array}{r}x=0 \\ y=0\end{array}\right.$ e $V_{2}:=\left\{\begin{array}{r}-3 x-2 t=0 \\ y=0\end{array}\right.$. Então, $V(\alpha) / W=V_{2}$.

Se $t=0, V(\alpha)$ tem apenas uma componente: $V_{1}:=\left\{\begin{array}{l}x=0 \\ y=0\end{array}\right.$.

Então, $V(\alpha) / W=\varnothing$.

O lema seguinte é muito útil para calcular $V / W$.

Lema 3.4. Seja $\left(X, \mathscr{O}_{X}\right)$ um espaço analítico complexo, sejam $\alpha, \beta$ e $\gamma$ feixes coerentes de ideais em $\mathscr{O}_{X}$, sejam $f, g \in \mathscr{O}_{X}$, e seja $W$ um subconjunto analítico de X. Então,

i) $(\alpha+\beta) / W=(\alpha / W+\beta) / W$, e assim, como espaços analíticos complexos, $(V(\alpha) \cap$ $V(\beta)) / W=(V(\alpha / W) \cap V(\beta)) / W$;

ii) $\operatorname{Se} V(\alpha+\gamma) \subseteq W$, então $((\alpha \cap \beta)+\gamma) / W=(\beta+\gamma) / W$, e assim, como espaços analíticos complexos, $((V(\alpha) \cup V(\beta)) \cap V(\gamma)) / W=(V(\beta) \cap V(\gamma)) / W$;

iii) Se $V(\alpha+g) \subseteq W$, então $(\alpha+f g) / W=(\alpha+f) / W$, e assim, como espaços analíticos complexos, $(V(\alpha) \cap V(f g)) / W=(V(\alpha) \cap V(f)) / W$.

Esses feixes lacunas são usados para definir as variedades polares relativas da função analítica $h:(U, 0) \rightarrow(\mathbb{C}, 0)$, onde $U$ é um subconjunto aberto de $\mathbb{C}^{n+1}$. As características fundamentais desta definição são que as variedades polares não são necessariamente reduzidas e que a dimensão do conjunto crítico de $h$ pode ser arbitrária.

Definição 3.5. Para $0 \leq k \leq n$, a $k$-ésima variedade polar (relativa), $\Gamma_{h, z}^{k}$, de $h$ com respeito a $z$ é o espaço analítico complexo $V\left(\frac{\partial h}{\partial z_{k}}, \ldots, \frac{\partial h}{\partial z_{n}}\right) / \Sigma(h)$. Se a escolha do sistema de coordenadas é clara, escrevemos simplesmente $\Gamma_{h}^{k}$.

Assim, ao nível de ideais, $\Gamma_{h, z}^{k}$ consiste das componentes de $V\left(\frac{\partial h}{\partial z_{k}}, \ldots, \frac{\partial h}{\partial z_{n}}\right)$ que não estão contidas em $|\Sigma(h)|$. Note, em particular, que $\Gamma_{h, z}^{0}$ é vazio. Naturalmente, definimos o $k$-ésimo ciclo polar de $h$ com respeito a $z$ sendo o ciclo analítico $\left[\Gamma_{h, z}^{k}\right]$. 
Claramente, temos a seguinte sequência entre esses conjuntos:

$$
\varnothing=\Gamma_{h, z}^{0} \subseteq \Gamma_{h, z}^{1} \subseteq \ldots \subseteq \Gamma_{h, z}^{n+1}=U
$$

\subsection{Ciclos de Lê e números de Lê}

Do Lema 3.4.i, temos que

Proposição 3.6. $\left(\Gamma_{h, z}^{k+1} \cap V\left(\frac{\partial h}{\partial z_{k}}\right)\right) / \Sigma(h)=\Gamma_{h, z}^{k}$ como espaço analítico complexo, e assim todas as componentes do ciclo $\left[\Gamma_{h, z}^{k+1} \cap V\left(\frac{\partial h}{\partial z_{k}}\right)\right]-\left[\Gamma_{h, z}^{k}\right]$ estão contidas no conjunto crítico da aplicação $h$.

Como o ideal $\left\langle\frac{\partial h}{\partial z_{k}}, \ldots, \frac{\partial h}{\partial z_{n}}\right\rangle$ é invariante sobre qualquer mudança linear de coordenadas que deixa $V\left(z_{0}, \ldots, z_{k-1}\right)$ invariante, vemos que $\Gamma_{h, z}^{k}$ depende somente de $h$ e da escolha das primeiras $k$-coordenadas. No momento, ele será conveniente para a subscrição da $k$-ésima variedade polar com somente as primeiras $k$ coordenadas ao invés daquele sistema de coordenadas. Por enquanto, escrevemos $\Gamma_{h, z_{0}}^{1}$ para a curva polar.

É imediato, do número de equações definidas, que toda componente isolada do conjunto analítico $\left|\Gamma_{h, z}^{k}\right|$ tem dimensão no mínimo $k$, na verdade queremos que o sistema de coordenadas seja adequadamente genérico de modo que $\Gamma_{h, z}^{k}$ tenha dimensão pura $k$.

Quando essa condição de dimensão é satisfeita, nosso objetivo é verificar que $\Gamma_{h, z}^{k}$ não tem componentes mergulhadas.

Para isso, necessitamos de dois lemas.

Lema 3.7. Seja $p \in V:=V\left(g_{1}, \ldots, g_{d}\right) \subseteq U$, onde $U$ é um subconjunto aberto de $\mathbb{C}^{n+1}$ e seja $W$ igual a união das componentes de $V$ através de p de dimensão maior que $n+1-d$. Então, $V / W$ não tem subvariedades mergulhadas através de p.

Demonstraçãa. Por definição, $V / W$ não tem componentes - mergulhadas ou isoladas contidas em $\mathrm{W}$. Assim, se $V / W$ tem uma componente mergulhada através de $p$, essa componente 
tem que conter pontos $q$, arbitrariamente próximos em $p$, que não estão contidos em $W$. Mas a localização de $V / W$ em qualquer ponto fora de $W$ é uma intersecção completa local e por isso não tem componentes mergulhadas. Isso contradiz a existência de uma componente mergulhada através de $q$.

Lema 3.8. Se $V:=V\left(g_{1}, \ldots, g_{d}\right)$ e $\operatorname{dim}_{p} V / Z=n+1-d$ então $V / Z$ não tem componentes mergulhadas através de $p$.

Demonstração. Na notação do Lema 3.7, isso segue do fato que $Z$ tem que conter $W$.

Corolário 3.9. Se $\operatorname{dim}_{p} \Gamma_{h, z}^{k}=k$, então $\Gamma_{h, z}^{k}$ não tem subvariedades mergulhadas através do ponto $p$.

Proposição 3.10. Se $\operatorname{dim}_{p} \Sigma(h)<k$, então $\Gamma_{h, z}^{k}=V\left(\frac{\partial h}{\partial z_{k}}, \ldots, \frac{\partial h}{\partial z_{n}}\right)$ como ciclo de p. Se, além disso, $\operatorname{dim}_{p} \Gamma_{h, z}^{k}=k$, então a igualdade acima mantem-se como espaço analítico complexo de $p$.

Demonstração. $V:=V\left(\frac{\partial h}{\partial z_{k}}, \ldots, \frac{\partial h}{\partial z_{n}}\right)$ consiste das componentes não contidas em $\Sigma(h)$ - esses constituem $\Gamma_{h, z}^{k}$ - juntos com aquelas contidas em $\Sigma(h)$. Pelo número de equações definidas, toda componente isolada de $V$ tem que ter dimensão no mínimo $k$.

Assim, se $\operatorname{dim}_{p} \Sigma(h)<k$, as componentes de $V$ que estão contidas em $\Sigma(h)$ tem que estar mergulhadas. Por isso, $V\left(\frac{\partial h}{\partial z_{k}}, \ldots, \frac{\partial h}{\partial z_{n}}\right)$ é igual a $\Gamma_{h, z}^{k}$ a menos de componentes mergulhadas e, daí, eles são iguais como ciclos.

Mas isso certamente implica que $\Gamma_{h, z}^{k}$ e $V$ são iguais como germes de conjuntos em $p$.

Assim, se $\operatorname{dim}_{p} \Gamma_{h, z}^{k}=k$ então $\operatorname{dim}_{p} V=k$, isto é, V é uma intersecção local completa de $p$. Daí, $V$ não tem componentes mergulhadas em $p$ e, portanto, $\Gamma_{h, z}^{k}=V\left(\frac{\partial h}{\partial z_{k}}, \ldots, \frac{\partial h}{\partial z_{n}}\right)$.

Definiçãa 3.11. Se a intersecção de $\Gamma_{h, z}^{k}$ e $V\left(z_{0}-p_{0}, \ldots, z_{k-1}-p_{k-1}\right)$ é puramente 0-dimensional no ponto $p=\left(p_{0}, \ldots, p_{n}\right)$ (isto é, cada p é um ponto isolado da intersecção ou p não está 
na intersecção), então dizemos que a k-ésima multiplicidade polar, $\gamma_{h, z}^{k}(p)$, está definida e a igualamos com o número de intersecções

$$
\left(\Gamma_{h, z}^{k} \cdot V\left(z_{0}-p_{0}, \ldots, z_{k-1}-p_{k-1}\right)\right)_{p}
$$

Note que, se $\gamma_{h, z}^{k}$ está definida em $p$, então está definida em todo ponto próximo de $p$. Note também que, se $\gamma_{h, z}^{k}$ está definida, então $\Gamma_{h, z}^{k}$ tem que ser puramente $k$-dimensional em $p$ e assim - por 3.9 - $\Gamma_{h, z}^{k}$ não tem componentes mergulhadas em $p$.

Observação 3.12. Como conjuntos,

$$
\begin{aligned}
\Sigma\left(h_{\mid V\left(z_{0}-p_{0}, \ldots, z_{k-1}-p_{k-1}\right)}\right) & =V\left(z_{0}-p_{0}, \ldots, z_{k-1}-p_{k-1}, \frac{\partial h}{\partial z_{k}}, \ldots, \frac{\partial h}{\partial z_{n}}\right) \\
& =V\left(z_{0}-p_{0}, \ldots, z_{k-1}-p_{k-1}\right) \cap\left(\Sigma(h) \cup \Gamma_{h, z}^{k}\right) .
\end{aligned}
$$

Daí, se $\gamma_{h, z}^{k}(p)$ está definida e $p \in \Sigma(h)$, então

$$
\Sigma\left(h_{\mid V\left(z_{0}-p_{0}, \ldots, z_{k-1}-p_{k-1}\right)}\right)=V\left(z_{0}-p_{0}, \ldots, z_{k-1}-p_{k-1}\right) \cap \Sigma(h) \text { em } p .
$$

Agora, desejamos definir os ciclos de Lê. Diferente das variedades e ciclos polares, os ciclos de Lê estão no conjunto crítico de $h$. Esses ciclos demonstram um número de propriedades que generalizam o número de Milnor numa singularidade isolada.

Definição 3.13. Para $0 \leq k \leq n$, definimos o $k$-ésimo ciclo de Lê de h com respeito a $z$, denotado $\operatorname{por}\left[\Lambda_{h, z}^{k}\right]$, como sendo

$$
\left[\Gamma_{h, z}^{k+1} \cap V\left(\frac{\partial h}{\partial z_{k}}\right)\right]-\left[\Gamma_{h, z}^{k}\right] .
$$

Se a escolha do sistema de coordenadas é clara, simplificaremos escrevendo $\left[\Lambda_{h}^{k}\right]$. Também, como temos dado os ciclos de Lê sem estrutura como espaço analítico complexo, omitiremos os colchetes e escreveremos $\Lambda_{h, z}^{k}$ para denotar o ciclo de Lê - a não ser que explicitemos que estamos considerando-o como um conjunto somente. 
Note que, como toda componente de $\Gamma_{h, z}^{k+1}$ tem dimensão no mínimo $k+1$, toda componente de $\Lambda_{h, z}^{k}$ tem dimensão no mínimo $k$.

Dizemos que o ciclo $\left[\Lambda_{h, z}^{k}\right]$ ou o conjunto $\left|\Lambda_{h, z}^{k}\right|$ tem dimensão própria em um ponto $p$ desde que $\left|\Lambda_{h, z}^{k}\right|$ seja puramente $k$-dimensional em $p$.

Definimos o $k$-ésimo número de Lê de $h$ em $p$ com respeito a $z, \lambda_{h, z}^{k}(p)$, como sendo igual ao número de intersecções

$$
\left(\Lambda_{h, z}^{k} \cdot V\left(z_{0}-p_{0}, \ldots, z_{k-1}-p_{k-1}\right)\right) p
$$

desde que essa intersecção seja 0-dimensional em $p$.

Se essa intersecção não é puramente 0 - dimensional em $p$, então dizemos que o $k$-ésimo número de Lê (de $h$ em $p$ com respeito a $z$ ) está indefinido.

Aqui, quando $k=0$, queremos dizer que

$$
\lambda_{h, z}^{0}(p)=\left(\Lambda_{h, z}^{0} \cdot U\right)_{p}=\left(\left[\Gamma_{h, z}^{1} \cap V\left(\frac{\partial h}{\partial z_{0}}\right)\right]\right)_{p}=\left(\left[\Gamma_{h, z}^{1}\right] \cdot\left[V\left(\frac{\partial h}{\partial z_{0}}\right)\right]\right)_{p}
$$

( Essa última igualdade mantem-se sempre que $\Gamma_{h, z}^{1}$ é 1-dimensional em $p$, por isso $\Gamma_{h, z}^{1}$ não tem componentes mergulhadas por 3.9 e $\Gamma_{h, z}^{1} \cap V\left(\frac{\partial h}{\partial z_{0}}\right)$ é 0-dimensional).

Note que se $\lambda_{h, z}^{k}(p)$ está definido, então $\lambda_{h, z}^{k}$ está definido em todos os pontos próximos de $p$.

Também note, desde que $\Gamma_{h, z}^{k+1}$ e $\Gamma_{h, z}^{k}$ dependam somente da escolha das coordenadas $z_{0}$ a $z_{k}$, o $k$-ésimo ciclo de Lê depende somente da escolha de $\left(z_{0}, \ldots, z_{k}\right)$. Finalmente, note que se $h$ é um polinômio, então desde que estamos tomando coordenadas lineares, permanecemos dentro da categoria algébrica.

Exemplo 3.14. Seja $h=y^{2}-x^{3}-t x^{2}$; esse é o guarda-chuva de Whitney do exemplo 2.8 . Fixamos o sistema de coordenadas $z=(t, x, y)$.

Temos $\Sigma(h)=V\left(-x^{2},-3 x^{2}-2 t x, 2 y\right)=V(x, y)$. Assim o conjunto crítico de $h$ é 1-dimensional, enquanto a dimensão de toda componente de $V\left(\frac{\partial h}{\partial y}\right)$ é 2 . 


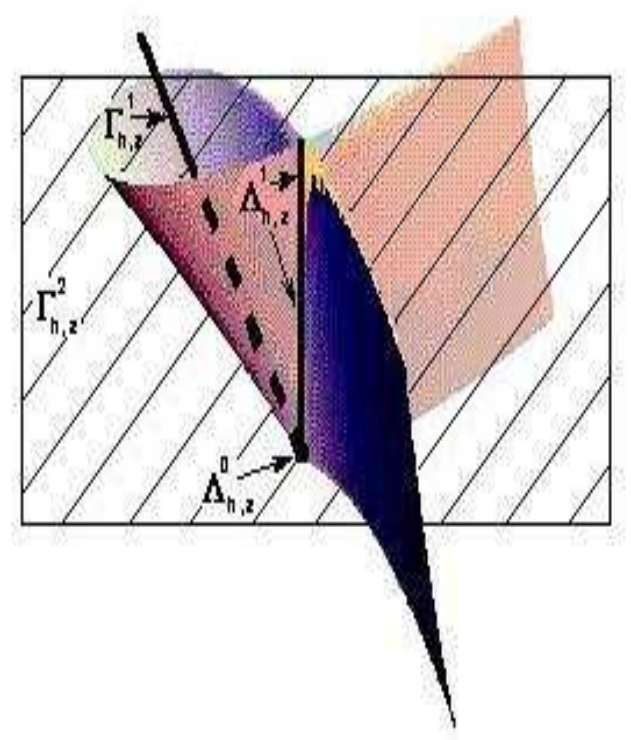

Figura 3.1: Ciclos Polares e Ciclos de Lê

Daí, $V\left(\frac{\partial h}{\partial y}\right)$ não possui nenhuma componente contida em $\Sigma(h)$ e, por isso, começamos calculando variedades polares com $\Gamma_{h}^{2}$.

Temos $\Gamma_{h}^{2}=V\left(\frac{\partial h}{\partial y}\right)=V(2 y)=V(y)$ e, portanto,

$$
\Gamma_{h}^{2} \cap V\left(\frac{\partial h}{\partial x}\right)=V(y) \cap V\left(-3 x^{2}-2 t x\right)=V\left(y,-3 x^{2}-2 t x\right) .
$$

Aplicando 3.6 e 3.4 iii, achamos

$$
\Gamma_{h}^{1}=\left(\Gamma_{h}^{2} \cap V\left(\frac{\partial h}{\partial x}\right)\right) / \Sigma(h)=V\left(y,-3 x^{2}-2 t x\right) / V(x, y)=V(y,-3 x-2 t) .
$$

Da definição do ciclo de Lê (3.13) obtemos

$$
\begin{aligned}
\Lambda_{h}^{1} & =\left[V\left(y,-3 x^{2}-2 t x\right)\right]-[V(y,-3 x-2 t)] \\
& =([V(y, x)]+[V(y,-3 x-2 t)])-[V(y,-3 x-2 t)] \\
& =[V(y, x)] .
\end{aligned}
$$

Assim, $\Lambda_{h}^{1}$ tem como seu conjunto básico o eixo $t$ e esse eixo ocorre com multiplicidade 1. 
Agora achamos

$$
\Lambda_{h}^{0}=\left[\Gamma_{h}^{1} \cap V\left(\frac{\partial h}{\partial t}\right)\right]=\left[V(y,-3 x-2 t) \cap V\left(-x^{2}\right)\right]=2[V(t, x, y)]=2[0] .
$$

Finalmente calculamos os números de Lê: $\lambda_{h}^{1}(0)=(V(y, x) \cdot V(t))_{0}=1$ e $\lambda_{h}^{0}(0)=2$.

\subsection{Propriedades dos ciclos de Lê}

Provaremos agora resultados gerais sobre ciclos de Lê e números de Lê. Em particular, queremos obter a Proposição 3.19, que fala como que, quando calculando ciclos de Lê, podemos trabalhar unicamente ao nível de ciclos, ao invés de nos preocuparmos com a estrutura da variedade polar como espaço analítico complexo.

Proposição 3.15. Os ciclos de Lê são todos não negativos e estão contidos no conjunto crítico de $h$. Toda componente de $\left|\Lambda_{h, z}^{k}\right|$ tem dimensão no mínimo $k$. Se $s:=\operatorname{dim}_{p} \Sigma(h)$ então, para $s<k<n+1$, p não está contido em $\left|\Lambda_{h, z}^{k}\right|$, isto é, $\left|\Lambda_{h, z}^{k}\right|$ é vazio em p; assim, para $s<k<n+1$, $\lambda_{h, z}^{k}(p)$ está definido e é igual a 0.

Demonstraçãa. A primeira sentença segue de 3.6. A segunda sentença segue da definição de ciclo de Lê e do fato que toda componente de $\Gamma_{h, z}^{k}$ tem dimensão no mínimo $k+1$. A terceira sentença segue das duas primeiras.

Devido a proposição acima, são considerados apenas $\lambda^{0}, \ldots, \lambda^{s}$.

Proposição 3.16. Como conjuntos, para todo $k, \Gamma_{h, z}^{k+1} \cap \Sigma(h)=\cup_{i \leq k} \Lambda_{h, z}^{i}$ Em particular, deixando $k=s:=\operatorname{dim}_{p} \Sigma(h)$, como germes de conjuntos em $p, \Sigma(h)=\cup_{i \leq k} \Lambda_{h, z}^{i}$.

Demonstração. Como conjuntos temos

$$
\begin{aligned}
\Gamma_{h, z}^{k+1} \cap \Sigma(h) & =\Gamma_{h, z}^{k+1} \cap V\left(\frac{\partial h}{\partial z_{k}}\right) \cap \Sigma(h)=\left(\Gamma_{h, z}^{k} \cup \Lambda_{h, z}^{k}\right) \cap \Sigma(h)=\left(\Gamma_{h, z}^{k} \cap \Sigma(h)\right) \cup \Lambda_{h, z}^{k} \\
& =\left(\left(\Gamma_{h, z}^{k-1} \cap \Sigma(h)\right) \cup \Lambda_{h, z}^{k-1}\right) \cup \Lambda_{h, z}^{k}=\cdots=\cup_{i \leq k} \Lambda_{h, z}^{i} .
\end{aligned}
$$


Da observação 3.12, se $\gamma_{h, z}^{1}(p)$ está definido e $p \in \Sigma(h)$, então

$$
\Sigma\left(h_{\mid V\left(z_{0}-p_{0}\right)}\right)=V\left(z_{0}-p_{0}\right) \cap \Sigma(h)
$$

em $p$. Esse é especialmente usado em provas de indução quando combinado com o seguinte resultado:

Proposição 3.17. Se $s:=\operatorname{dim}_{p} \Sigma(h) \geq 1, \Lambda_{h, z}^{i}$ tem dimensão própria em p para todo $i \leq s-$ 1 (quer dizer que p não está contido em nenhum dos $\left.\Lambda_{h, z}^{i}{ }^{\prime} s\right)$ e $\lambda_{h, z}^{s}(p)$ está definido, então $\operatorname{dim}_{p}\left(\Sigma(h) \cap V\left(z_{0}-p_{0}\right)\right)=s-1$.

Demonstração. Como estamos assumindo que $\Lambda_{h, z}^{i}$ tem dimensão própria em $p$ para todo $i \leq s-1$, segue de 3.16 que é suficiente mostrar que a fatia do hiperplano $V\left(z_{0}-p_{0}\right)$ reduz a dimensão de $\Lambda_{h, z}^{s}$. Mas, esse tem que ser o caso, desde que

$$
\Lambda_{h, z}^{s} \cap V\left(z_{0}-p_{0}, \ldots, z_{s-1}-p_{s-1}\right)
$$

é 0-dimensional em $p$.

Necessitamos do seguinte lema, que é uma consequência imediata de 7.1.b de [4].

Lema 3.18. Se uma hipersuperfície $V(h)$ não contem componentes, mergulhadas ou isoladas, de um espaço analítico complexo $V(\alpha)$ através de p, então os ciclos $[V(\alpha)] \cdot[V(h)]$ e $[V(\alpha) \cap V(h)]$ são iguais em $p$.

Proposição 3.19. Se, para todo $j$ com $0 \leq j \leq k, \Lambda_{h, z}^{j}$ é puramente j-dimensional em p, então $\Gamma_{h, z}^{k+1} \cap V\left(\frac{\partial h}{\partial z_{k}}\right)$ é puramente $k$-dimensional em $p$, e também $\Gamma_{h, z}^{k+1}$ é puramente $(k+1)$ dimensional em $p$ e os ciclos

$$
\left[\Gamma_{h, z}^{k+1} \cap V\left(\frac{\partial h}{\partial z_{k}}\right)\right] e\left[\Gamma_{h, z}^{k+1}\right] \cdot\left[V\left(\frac{\partial h}{\partial z_{k}}\right)\right]
$$

são iguais em p. Além disso, nesse caso, toda componente k-dimensional (isolada) do conjunto crítico de hatravés de p está contida em $\left|\Lambda_{h, z}^{k}\right|$. 
Demonstração. A demonstração da primeira sentença é por indução sobre $k$.

Para $k=0$, a sentença é trivialmente vista ser verdade pois $\left[\Lambda_{h, z}^{0}\right]=\left[\Gamma_{h, z}^{1} \cap V\left(\frac{\partial h}{\partial z_{0}}\right)\right]$.

Assuma a hipótese para $k-1$ e suponha que $\Lambda_{h, z}^{j}$ é puramente $j$-dimensional em $p$ para todo $0 \leq j \leq k$. Pela hipótese indutiva, $\Gamma_{h, z}^{k}$ é puramente $k$-dimensional em $p$.

Desde que, como conjuntos $\Gamma_{h, z}^{k+1} \cap V\left(\frac{\partial h}{\partial z_{k}}\right)=\Gamma_{h, z}^{k} \cup \Lambda_{h, z}^{k}$ e estamos assumindo que $\Lambda_{h, z}^{k}$ é puramente $k$-dimensional em $p$, temos que $\Gamma_{h, z}^{k+1} \cap V\left(\frac{\partial h}{\partial z_{k}}\right)$ é puramente $k$-dimensional em $p$.

Agora, assuma que $\Gamma_{h, z}^{k+1} \cap V\left(\frac{\partial h}{\partial z_{k}}\right)$ é puramente $k$-dimensional em $p$. Então $\Gamma_{h, z}^{k+1}$ é puramente $(k+1)$-dimensional em $p$ e $V\left(\frac{\partial h}{\partial z_{k}}\right)$ não contem componentes (isoladas) de $\Gamma_{h, z}^{k+1}$ através de $p$.

Agora, pela Proposição 3.9, desde que $\Gamma_{h, z}^{k+1}$ é puramente $(k+1)$-dimensional em $p, \Gamma_{h, z}^{k+1}$ não tem componentes mergulhadas através de $p$. Assim, $V\left(\frac{\partial h}{\partial z_{k}}\right)$ não contem componentes isoladas ou mergulhadas - de $\Gamma_{h, z}^{k+1}$ através de $p$, e daí pelo Lema 3.18, concluímos que os ciclos

$$
\left[\Gamma_{h, z}^{k+1} \cap V\left(\frac{\partial h}{\partial z_{k}}\right)\right] e\left[\Gamma_{h, z}^{k+1}\right] \cdot\left[V\left(\frac{\partial h}{\partial z_{k}}\right)\right]
$$

são iguais em $p$.

Finalmente, seja $C$ uma componente $k$-dimensional de $|\Sigma(h)|$. Então, $C \subseteq C^{\prime}$, onde $C^{\prime}$ é uma componente de $\left|V\left(\frac{\partial h}{\partial z_{k+1}}, \ldots, \frac{\partial h}{\partial z_{n}}\right)\right|$. Agora, como $C$ é uma componente de $|\Sigma(h)| \mathrm{e}$ $\operatorname{dim} C^{\prime} \geq k+1$, concluímos que $C^{\prime} \nsubseteq|\Sigma(h)|$, isto é, $C^{\prime} \subseteq\left|\Gamma_{h, z}^{k+1}\right|$. Por isso, como conjuntos,

$$
C=C \cap V\left(\frac{\partial h}{\partial z_{k}}\right) \subseteq \Gamma_{h, z}^{k+1} \cap V\left(\frac{\partial h}{\partial z_{k}}\right)=\Gamma_{h, z}^{k} \cup \Lambda_{h, z}^{k}
$$

A conclusão agora segue da primeira parte da proposição.

Na prática, usamos a primeira parte da Proposição 3.19 para calcular os ciclos de Lê como segue:

Assuma, no momento, que todos os ciclos de Lê têm dimensão própria, e seja $s$ a dimensão 
do conjunto crítico de $h$. Então,

$$
\begin{gathered}
{\left[\Gamma_{h, z}^{s+1}\right]=\left[V\left(\frac{\partial h}{\partial z_{s+1}}, \ldots, \frac{\partial h}{\partial z_{n}}\right)\right] .} \\
{\left[\Gamma_{h, z}^{s+1}\right] \cdot\left[V\left(\frac{\partial h}{\partial z_{s}}\right)\right]=\left[\Gamma_{h, z}^{s}\right]+\left[\Lambda_{h, z}^{s}\right] .} \\
{\left[\Gamma_{h, z}^{s}\right] \cdot\left[V\left(\frac{\partial h}{\partial z_{s-1}}\right)\right]=\left[\Gamma_{h, z}^{s-1}\right]+\left[\Lambda_{h, z}^{s-1}\right] .} \\
\left.\vdots \Gamma_{h, z}^{2}\right] \cdot\left[V\left(\frac{\partial h}{\partial z_{1}}\right)\right]=\left[\Gamma_{h, z}^{1}\right]+\left[\Lambda_{h, z}^{1}\right] . \\
{\left[\Gamma_{h, z}^{1}\right] \cdot\left[V\left(\frac{\partial h}{\partial z_{0}}\right)\right]=\left[\Lambda_{h, z}^{0}\right] .}
\end{gathered}
$$

Em cada linha acima, $\left[\Gamma_{h, z}^{k}\right]$ é usado para o cálculo da próxima linha. Agora, para escrever as equações anteriores, tivemos que usar que os ciclos de Lê têm dimensão própria - mas, em qualquer caso, as equações são verdadeiras como conjuntos (usando intersecção e união de conjuntos). E assim, depois de fazer os cálculos anteriores, verifica-se que os ciclos de Lê que têm dimensão própria, as equações estão corretas. Por outro lado, se os ciclos de Lê não têm dimensão própria, as equações anteriores podem ser falsas.

\subsection{Exemplos}

O exemplo a seguir mostra que, no caso de singularidade isolada, o número de Lê $\lambda_{h, z}^{0}$ é o número de Milnor de $h$ em 0.

Exemplo 3.20. Se 0 é uma singularidade isolada de $h$, então indiferentemente do sistema de coordenadas $z$, segue da Proposição 3.15 que a única possibilidade não nula do número de Lê é $\lambda_{h, z}^{0}(0)$. Além disso, como $V\left(\frac{\partial h}{\partial z_{0}}, \ldots, \frac{\partial h}{\partial z_{n}}\right)$ é 0 -dimensional, $V\left(\frac{\partial h}{\partial z_{1}}, \ldots, \frac{\partial h}{\partial z_{n}}\right)$ é 
1-dimensional sem componentes contidas em $\Sigma(h)$ e sem componentes mergulhadas. Então,

$$
\Gamma_{h, z}^{1}=V\left(\frac{\partial h}{\partial z_{1}}, \ldots, \frac{\partial h}{\partial z_{n}}\right)
$$

e assim,

$$
\begin{aligned}
\lambda_{h, z}^{0} & =\left(\Gamma_{h, z}^{1} \cdot V\left(\frac{\partial h}{\partial z_{0}}\right)\right)_{0}=\left(V\left(\frac{\partial h}{\partial z_{1}}, \ldots, \frac{\partial h}{\partial z_{n}}\right) \cdot V\left(\frac{\partial h}{\partial z_{0}}\right)\right)_{0} \\
& =\left[V\left(\frac{\partial h}{\partial z_{0}}, \ldots, \frac{\partial h}{\partial z_{n}}\right)\right]_{0}=\text { o número de Milnor de } h \text { em } 0 .
\end{aligned}
$$

Exemplo 3.21. Aqui, generalizamos o exemplo 3.14. Seja $h=y^{2}-x^{a}-t x^{b}$, onde $a>b>1$. Fixamos o sistema de coordenadas $z=(t, x, y)$.

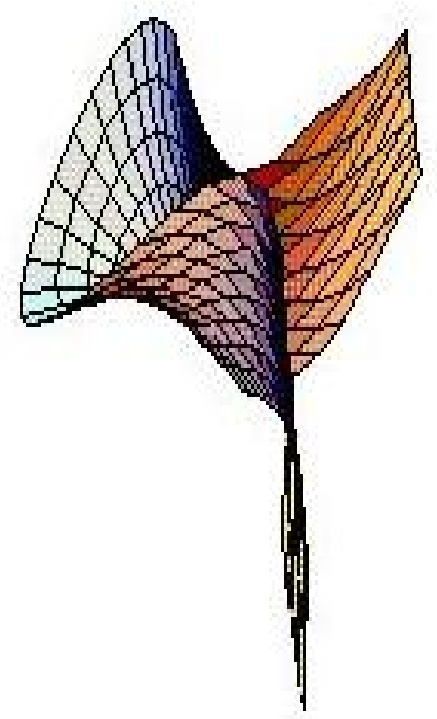

Figura 3.2: Generalização de nós que se degeneram em uma cúspide quando $a=3$ e $b=2$

$$
\begin{gathered}
\Sigma(h)=V\left(-x^{b},-a x^{a-1}-b t x^{b-1}, 2 y\right)=V(x, y) . \\
\Gamma_{h}^{2}=V\left(\frac{\partial h}{\partial y}\right)=V(2 y)=V(y) .
\end{gathered}
$$




$$
\begin{aligned}
& \Gamma_{h}^{2} \cdot V\left(\frac{\partial h}{\partial x}\right)=V(y) \cdot V\left(-a x^{a-1}-b t x^{b-1}\right) \quad=V(y) \cdot\left(V\left(-a x^{a-b}-b t\right)+V\left(x^{b-1}\right)\right) \\
& =V\left(-a x^{a-b}-b t, y\right)+(b-1) V(x, y)=\Gamma_{h}^{1}+\Lambda_{h}^{1} . \\
& \Gamma_{h}^{1} \cdot V\left(\frac{\partial h}{\partial t}\right)=V\left(-a x^{a-b}-b t, y\right) \cdot V\left(-x^{b}\right)=b V(t, x, y)=b[0]=\Lambda_{h}^{0}
\end{aligned}
$$

Assim, $\lambda_{h}^{0}(0)=b$ e $\lambda_{h}^{1}(0)=b-1$.

Observamos que neste exemplo, o expoente $a$ não interfere nos cálculos. Isso deve-se ao fato de que $h=y^{2}-x^{a}-t x^{b}=y^{2}-x^{b}\left(x^{a-b}-t\right)$ que é, depois de uma mudança de coordenadas na origem, igual a $y^{2}-x^{b} u$.

Exemplo 3.22. Seja $h=y^{2}-x^{3}-\left(u^{2}+v^{2}+w^{2}\right) x^{2}$. Fixamos o sistema de coordenadas $z=$ $(u, v, w, x, y)$.

$$
\Sigma(h)=V\left(-2 u x^{2},-2 v x^{2},-2 w x^{2},-3 x^{2}-2 x\left(u^{2}+v^{2}+w^{2}\right), 2 y\right)=V(x, y) .
$$

Como $\Sigma(h)$ é 3-dimensional, começamos nosso cálculo com $\Gamma_{h}^{4}$.

$$
\begin{gathered}
\Gamma_{h}^{4}=V(-2 y)=V(y) . \\
\Gamma_{h}^{4} \cdot V\left(\frac{\partial h}{\partial x}\right)=V(y) \cdot V\left(-3 x^{2}-2 x\left(u^{2}+v^{2}+w^{2}\right)\right) \\
=V\left(-3 x-2\left(u^{2}+v^{2}+w^{2}\right), y\right)+V(x, y) \\
=\Gamma_{h}^{3}+\Lambda_{h}^{3} . \\
\Gamma_{h}^{3} \cdot V\left(\frac{\partial h}{\partial w}\right)=V\left(-3 x-2\left(u^{2}+v^{2}+w^{2}\right), y\right) \cdot V\left(-2 w x^{2}\right) \\
=\quad V\left(-3 x-2\left(u^{2}+v^{2}\right), w, y\right)+2 V\left(u^{2}+v^{2}+w^{2}, x, y\right) \\
=\Gamma_{h}^{2}+\Lambda_{h}^{2} . \\
=\quad V\left(-3 x-2\left(u^{2}+v^{2}\right), w, y\right) \cdot V\left(-2 v x^{2}\right) \\
=\quad V\left(-3 x-2 u^{2}, v, w, y\right)+2 V\left(u^{2}+v^{2}, w, x, y\right) \\
=\Gamma_{h}^{1}+\Lambda_{h}^{1} .
\end{gathered}
$$




$$
\begin{aligned}
\Gamma_{h}^{1} \cdot V\left(\frac{\partial h}{\partial u}\right) & =V\left(-3 x-2 u^{2}, v, w, y\right) \cdot V\left(-2 u x^{2}\right) \\
& =V(u, v, w, x, y)+2 V\left(u^{2}, v, w, x, y\right)=5[0] \\
& =\Lambda_{h}^{0}
\end{aligned}
$$

Consequentemente, $\Lambda_{h}^{3}=V(x, y), \Lambda_{h}^{2}=2 V\left(u^{2}+v^{2}+w^{2}, x, y\right)$ (um cone, como conjunto), $\Lambda_{h}^{1}=2 V\left(u^{2}+v^{2}, w, x, y\right)$ e $\Lambda_{h}^{0}=5[0]$. Assim, na origem, $\lambda_{h}^{3}=1, \lambda_{h}^{2}=4, \lambda_{h}^{1}=4$ e $\lambda_{h}^{0}=5$.

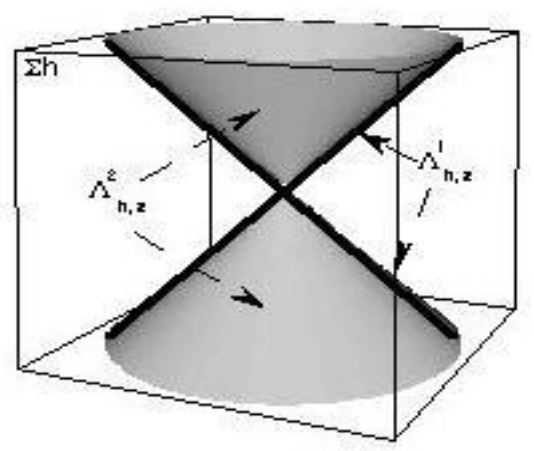

Figura 3.3: O conjunto crítico de $h$

Observamos que neste exemplo, a determinação dos ciclos de Lê dependem da escolha das coordenadas fixadas.

\subsection{Números de Lê e seções hiperplanas}

Na próxima proposição é mostrado como os números de Lê comportam-se tomando seções de hiperplanos - um resultado fundamental, que é combinação das Proposições 2.5, 2.6 e 2.9 de [13];

Proposição 3.23. Suponha $\Sigma(h) \cap V\left(z_{0}-p_{0}\right)=\Sigma\left(h_{\left.\right|_{V\left(z_{0}-p_{0}\right)}}\right)$ e use as coordenadas $\tilde{z}=\left(z_{1}, \ldots, z_{n}\right)$ para $V\left(z_{0}-p_{0}\right)$. Seja $k \geq 1$ e suponha que $\gamma_{h, z}^{k}(p) e \lambda_{h, z}^{k}(p)$ estão definidos. Então, $\gamma_{\left.\right|_{V\left(z_{0}-p_{0}\right)} ^{k-1}, \tilde{z}}(p)$ $e \lambda_{h_{V\left(z_{0}-p_{0}\right)}}^{k-1}, \tilde{z}(p)$ estão definidos, $e$

$$
\Gamma_{h_{\mid V\left(z_{0}-p_{0}\right)}^{k}, \tilde{z}}^{k}=\Gamma_{h, z}^{k+1} \cdot V\left(z_{0}-p_{0}\right)
$$




$$
\Gamma_{h_{V\left(z_{0}-p_{0}\right)}^{k-1}, \tilde{z}}^{k-\Lambda_{h_{V\left(z_{0}-p_{0}\right)}, z}^{k-1}}=\left(\Gamma_{h, z}^{k}+\Lambda_{h, z}^{k}\right) \cdot V\left(z_{0}-p_{0}\right)
$$

$e$

$$
\gamma_{h_{V\left(z_{0}-p_{0}\right)}^{k-1}, \tilde{z}}^{k}(p)+\lambda_{h_{V\left(z_{0}-p_{0}\right)}^{k-1}, \tilde{z}}^{k-1}(p)=\gamma_{h, z}^{k}(p)+\lambda_{h, z}^{k}(p)
$$

Em particular, quando $k=1$, segue que se $\gamma_{h, z}^{1}(p)$ e $\lambda_{h, z}^{1}(p)$ estão definidos, então $\lambda_{h_{V\left(z_{0}-p_{0}\right)}^{0}, \tilde{z}}(p)$ também está, $e$

$$
\lambda_{h_{V\left(z_{0}-p_{0}\right)}^{0}, \tilde{z}}^{0}(p)=\gamma_{h, z}^{1}(p)+\lambda_{h, z}^{1}(p) .
$$

Além disso, se $k \geq 1$ e $\gamma_{h, z}^{k}(p), \lambda_{h, z}^{k}(p), \gamma_{h, z}^{k+1}(p)$ e $\lambda_{h, z}^{k+1}(p)$ estão definidos, então $\gamma_{h_{V\left(z_{0}-p_{0}\right)}^{k-1}, \tilde{z}}(p), \lambda_{h_{V\left(z_{0}-p_{0}\right)}^{k-1}, \tilde{z}}^{k}(p), \gamma_{h_{V\left(z_{0}-p_{0}\right)}^{k}, \tilde{z}}^{k}(p) e \lambda_{\left.\right|_{V\left(z_{0}-p_{0}\right)} ^{k}, \tilde{z}}^{k}(p)$ também estão, $e$

$$
\begin{gathered}
\Gamma_{h_{V\left(z_{0}-p_{0}\right)}, \tilde{z}}^{k}=\Gamma_{h, z}^{k+1} \cdot V\left(z_{0}-p_{0}\right), \\
\Lambda_{h_{V\left(z_{0}-p_{0}\right)}, \tilde{z}}^{k}=\Lambda_{h, z}^{k+1} \cdot V\left(z_{0}-p_{0}\right),
\end{gathered}
$$

e assim

$$
\begin{aligned}
& \gamma_{\left.\right|_{V\left(z_{0}-p_{0}\right)} ^{k}, \tilde{z}}^{k}(p)=\gamma_{h, z}^{k+1}(p), \\
& \lambda_{h_{V\left(z_{0}-p_{0}\right)}^{k}, \tilde{z}}^{k}(p)=\lambda_{h, z}^{k+1}(p) .
\end{aligned}
$$

Demonstração. É suficiente demonstrar a afirmação para $p=0$. A suposição que $\gamma_{h, z}^{k}(0)$ e $\lambda_{h, z}^{k}(0)$ estão definidos é equivalente a

$$
\operatorname{dim}_{0}\left(\Gamma_{h, z}^{k+1} \cap V\left(\frac{\partial h}{\partial z_{k}}\right) \cap V\left(z_{0}, \ldots, z_{k-1}\right)\right) \leq 0 .
$$

Daí, $\Gamma_{h, z}^{k+1}$ é puramente $(k+1)$-dimensional na origem e assim não tem componentes mergulhadas (Proposição 3.9). 
Também, $\Gamma_{h, z}^{k+1} \cap V\left(\frac{\partial h}{\partial z_{k}}\right)$ é puramente $k$-dimensional na origem e assim, pelo Lema 3.18, é obtida a igualdade de ciclos

$$
\left[\Gamma_{h, z}^{k+1} \cap V\left(\frac{\partial h}{\partial z_{k}}\right)\right]=\Gamma_{h, z}^{k+1} \cdot V\left(\frac{\partial h}{\partial z_{k}}\right)=\Gamma_{h, z}^{k}+\Lambda_{h, z}^{k}
$$

Além disso, vimos que $\Gamma_{h, z}^{k+1} \cap V\left(\frac{\partial h}{\partial z_{k}}\right) \cap V\left(z_{0}\right)$ é puramente $(k-1)$-dimensional na origem; Concluímos que

$$
\operatorname{dim}_{0}\left(\Gamma_{h, z}^{k+1} \cap \Sigma(h) \cap V\left(z_{0}\right)\right) \leq k-1 .
$$

Agora, considere o ciclo $\Gamma_{h_{\mid V\left(z_{0}\right)}}^{k}, \tilde{z}$. Por definição,

$$
\Gamma_{h_{V\left(z_{0}\right)}, z}^{k}=V\left(z_{0}, \frac{\partial h}{\partial z_{k+1}}, \ldots, \frac{\partial h}{\partial z_{n}}\right) / \Sigma\left(h_{\left.\right|_{V\left(z_{0}\right)}}\right) .
$$

Usando o Lema 3.4 ii e a hipótese que $\Sigma(h) \cap V\left(z_{0}\right)=\Sigma\left(h_{\left.\right|_{V\left(z_{0}\right)}}\right)$, da igualdade anterior concluímos que

$$
\Gamma_{h_{\mid\left(z_{0}\right)}, \tilde{z}}^{k}=\left(V\left(z_{0}\right) \cap \Gamma_{h, z}^{k+1}\right) /\left(\Sigma(h) \cap V\left(z_{0}\right)\right)=\left(V\left(z_{0}\right) \cap \Gamma_{h, z}^{k+1}\right) / \Sigma(h) .
$$

Porém, $V\left(z_{0}\right) \cap \Gamma_{h, z}^{k+1}$ é puramente $k$-dimensional na origem e $\operatorname{dim}_{0}\left(\Gamma_{h, z}^{k+1} \cap \Sigma(h) \cap V\left(z_{0}\right)\right) \leq$ $k-1$. Por isso, $\Sigma(h)$ não contem componentes isoladas de $V\left(z_{0}\right) \cap \Gamma_{h, z}^{k+1}$ e assim, como ciclos,

$$
\begin{gathered}
\Gamma_{h_{\mid V\left(z_{0}\right)}, z}^{k}=\Gamma_{h, z}^{k+1} \cap V\left(z_{0}\right)=\Gamma_{h, z}^{k+1} \cdot V\left(z_{0}\right) . \\
\Gamma_{h_{V\left(z_{0}\right)}, z}^{k-1}+\Lambda_{h_{\mid V\left(z_{0}\right)}, z}^{k-1}=\Gamma_{h_{\mid V\left(z_{0}\right)}, z}^{k} \cdot V\left(\frac{\partial h}{\partial z_{k}}\right)=\Gamma_{h, z}^{k+1} \cdot V\left(z_{0}\right) \cdot V\left(\frac{\partial h}{\partial z_{k}}\right)=\left(\Gamma_{h, z}^{k}+\Lambda_{h, z}^{k}\right) \cdot V\left(z_{0}\right) .
\end{gathered}
$$

Que $\gamma_{\left.h_{V\left(z_{0}\right)}\right), \tilde{z}}^{k-1}(0)$ e $\lambda_{h_{V\left(z_{0}\right)}^{k-1}, \tilde{z}}^{k-1}(0)$ estão definidos e que $\gamma_{h_{V\left(z_{0}\right)}^{k-1}}^{k-z_{z}}(0)+\lambda_{h_{V\left(z_{0}\right)}, \tilde{z}^{-1}}^{k-1}(0)=\gamma_{h, z}^{k}(0)+$ $\lambda_{h, z}^{k}(0)$ segue da intersecção do ciclo $V\left(z_{1}, \ldots, z_{n}\right)$ com cada lado da equação anterior.

Analogamente, as outras igualdades são verificadas.

Corolário 3.24. Seja $k \geq 0$. Suponha $\Sigma(h) \cap V\left(z_{0}-p_{0}, \ldots, z_{k}-p_{k}\right)=\Sigma\left(h_{\left.\right|_{V\left(z_{0}-p_{0}, \ldots, z_{k}-p_{k}\right)}}\right)$, e que $\gamma_{h, z}^{i}(p)$ e $\lambda_{h, z}^{i}(p)$ estão definidos para todo $i \leq k$. Então, $\gamma_{h, z}^{k+1}(p)$ está definido. 
Demonstração. Novamente, demostramos a afirmação para $p=0$.

Caso 1: Se $0 \notin \Sigma(h)$, então próximo de $0, \Gamma_{h, z}^{k+1}=V\left(\frac{\partial h}{\partial z_{k+1}}, \ldots, \frac{\partial h}{\partial z_{n}}\right)$ e assim

$$
\Gamma_{h, z}^{k+1} \cap V\left(z_{0}, \ldots, z_{k}\right)=\Sigma\left(h_{\left.\right|_{\left(z_{0}, \ldots, z_{k}\right)}}\right)=\Sigma(h) \cap V\left(z_{0}, \ldots, z_{k}\right)=\varnothing
$$

Daí, $\gamma_{h, z}^{k+1}(0)$ está definido e é igual a zero.

Caso 2: $0 \in \Sigma(h)$. A demonstração é por indução em $k$.

Para $k=0$, a exigência é que se $0 \in \Sigma(h), \lambda_{h, z}^{0}(0)$ está definido, e $\Sigma(h) \cap V\left(z_{0}\right)=\Sigma\left(h_{\left.\right|_{V\left(z_{0}\right)}}\right)$, então $\operatorname{dim}_{0}\left(\Gamma_{h, z}^{1} \cap V\left(z_{0}\right)\right) \leq 0$.

Como $0 \in \Sigma(h)$ e $\lambda_{h, z}^{0}(0)$ está definido, devemos ter que $\operatorname{dim}_{0} \Gamma_{h, z}^{1} \leq 1$. Assim, se $\operatorname{dim}_{0} \Gamma_{h, z}^{1} \cap$ $V\left(z_{0}\right) \geq 1$, então $V\left(z_{0}\right)$ deve conter uma componente de $\Gamma_{h, z}^{1}$ através da origem. Mas, desde que $\Sigma(h) \cap V\left(z_{0}\right)=\Sigma\left(h_{\left.\right|_{V\left(z_{0}\right)}}\right)$,

$$
\Gamma_{h, z}^{1} \cap V\left(z_{0}\right) \subseteq V\left(z_{0}, \frac{\partial h}{\partial z_{1}}, \ldots, \frac{\partial h}{\partial z_{n}}\right)=V\left(z_{0}, \frac{\partial h}{\partial z_{0}}, \ldots, \frac{\partial h}{\partial z_{n}}\right)
$$

Daí, qualquer componente de $\Gamma_{h, z}^{1}$ contida em $V\left(z_{0}\right)$ também deve estar contida em $\Sigma(h)$; isso contradiz a definição de $\Gamma_{h, z}^{1}$.

Suponha agora que o corolário é verdadeiro até $k-1$, onde $k \geq 1$. Suponha $0 \in \Sigma(h), \Sigma(h) \cap$ $V\left(z_{0}, \ldots, z_{k}\right)=\Sigma\left(h_{\left.\right|_{V\left(z_{0}, \ldots, z_{k}\right)}}\right)$ e que $\gamma_{h, z}^{i}(0)$ e $\lambda_{h, z}^{i}(0)$ estão definidos para todo $i \leq k$.

Como $0 \in \Sigma(h)$ e $\gamma_{h, z}^{i}(0)$ está definido para todo $i \leq k$, da Observação 3.12 implica que $\Sigma(h) \cap V\left(z_{0}, \ldots, z_{i}\right)=\Sigma\left(h_{\left.\right|_{V\left(z_{0}, \ldots, z_{i}\right)}}\right)$ para todo $i \leq k-1$. Em particular, como $k \geq 1, \Sigma(h) \cap V\left(z_{0}\right)=$ $\Sigma\left(h_{\left.\right|_{V\left(z_{0}\right)}}\right)$.

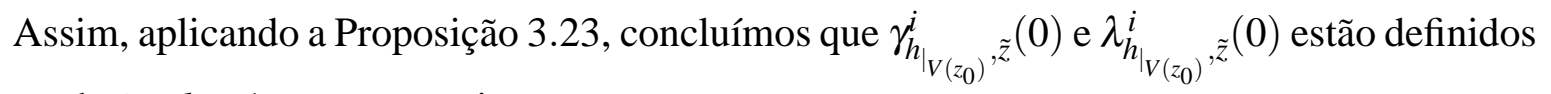
para todo $i \leq k-1$ e, como conjuntos,

$$
\Gamma_{h_{V\left(z_{0}\right)}, \tilde{z}}^{k}=\Gamma_{h, z}^{k+1} \cap V\left(z_{0}\right)
$$

Desde que $\Sigma\left(h_{\left.\right|_{V\left(z_{0}\right)}}\right) \cap V\left(z_{1}, \ldots, z_{k}\right)=\Sigma\left(h_{\left.\right|_{V\left(z_{0}, \ldots, z_{k}\right)}}\right)$, aplicando a hipótese indutiva para $h_{\left.\right|_{V\left(z_{0}\right)}}$, concluímos que $\gamma_{h_{V\left(z_{0}\right)}^{k}, \tilde{z}}(0)$ está definido, isto é, $\operatorname{dim}_{0}\left(\Gamma_{h_{\left.\right|_{V\left(z_{0}\right)}}^{k}, \tilde{z}} \cap V\left(z_{1}, \ldots, z_{k}\right)\right) \leq 0$. 
Como $\Gamma_{h_{\mid\left(z_{0}\right)}, \tilde{z}}^{k}=\Gamma_{h, z}^{k+1} \cap V\left(z_{0}\right)$, o resultado segue.

Necessitamos da seguinte relação entre três números de intersecções.

Proposição 3.25. Seja $p \in \Sigma(h)$. Então, $\lambda_{h, z}^{0}(p)$ está definido se, e somente se, $\operatorname{dim}_{p} \Gamma_{h, z}^{1} \leq 1$. Além disso, se $\gamma_{h, z}^{1}(p)$ está definido, então $\lambda_{h, z}^{0}(p)$ está definido, $\operatorname{dim}_{p}\left(\Gamma_{h, z}^{1} \cap V(h-h(p))\right) \leq 0$ $e$

$$
\left(\Gamma_{h, z}^{1} \cdot V(h-h(p))\right)_{p}=\lambda_{h, z}^{0}(p)+\gamma_{h, z}^{1}(p) .
$$

Demonstração. $\Gamma_{h, z}^{1}$ consiste das componentes de $V\left(\frac{\partial h}{\partial z_{1}}, \ldots, \frac{\partial h}{\partial z_{n}}\right)$ que não estão contidas em $|\Sigma(h)|$. Assim, $V\left(\frac{\partial h}{\partial z_{0}}\right)$ não contem componentes de $\Gamma_{h, z}^{1}$. Por isso, $\Gamma_{h, z}^{1}$ é puramente 1dimensional em $p$ se, e somente se, $\Gamma_{h, z}^{1} \cap V\left(\frac{\partial h}{\partial z_{0}}\right)$ é puramente 0 -dimensional em $p$, isto é, se, e somente se, $\lambda_{h, z}^{0}(p)$ está definido.

Se $\gamma_{h, z}^{1}(p)$ está definido, então $\Gamma_{h, z}^{1}$ é puramente 1-dimensional em $p$ e assim $\lambda_{h, z}^{0}(p)$ está definido.

Agora seguimos a demonstração na Proposição 1.3 de [12] para mostrar que uma aplicação da regra da cadeia produz $\operatorname{dim}_{p}\left(\Gamma_{h, z}^{1} \cap V(h-h(p))\right) \leq 0$ e $\left(\Gamma_{h, z}^{1} \cdot V(h-h(p))\right)_{p}=\lambda_{h, z}^{0}(p)+$ $\gamma_{h, z}^{1}(p)$.

Por conveniência, assuma que $p=0$ e que $h(0)=0$.

Suponha $\Gamma_{h, z}^{1}=\sum m_{W}[W]$ como ciclos. Podemos calcular o número de intersecções entre uma curva e uma hipersuperfície parametrizando a curva e achando a multiplicidade da composição da função definida pela hipersuperfície com a parametrização. Assim, para cada componente $W$, tome uma parametrização analítica local $\alpha(t)$ de $W$ tal que $\alpha(0)=0$.

Devemos mostrar duas coisas: que $h(\alpha(t))$ não é identicamente nula e que

$$
\text { mult }_{t} h(\alpha(t))=\text { mult }_{t}\left(\frac{\partial h}{\partial z_{0}}\right)_{\left.\right|_{\alpha(t)}}+\text { mult }_{t} z_{0}(\alpha(t)) \text {. }
$$

Como sabemos que o lado direito da igualdade acima é finito, temos somente que mostrar que a igualdade se mantem para concluir que $h(\alpha(t))$ não é identicamente nula. 


$$
\text { mult }_{t} h(\alpha(t))=1+\text { mult }_{t} \frac{d}{d t}\{h(\alpha(t))\}=1+\operatorname{mult}_{t}\left\{\left(\frac{\partial h}{\partial z_{0}}\right)_{\left.\right|_{\alpha(t)}} \cdot \alpha_{0}^{\prime}(t)\right\},
$$

onde os termos restantes que vem da regra da cadeia são zero desde que $\alpha(t)$ parametrize uma componente da curva polar.

Assim,

$$
\begin{aligned}
\text { mult }_{t} h(\alpha(t)) & =1+\text { mult }_{t}\left(\frac{\partial h}{\partial z_{0}}\right)_{\left.\right|_{\alpha(t)}}+\text { mult }_{t}\left(\alpha_{0}^{\prime}(t)\right) \\
& =\text { mult }_{t}\left(\frac{\partial h}{\partial z_{0}}\right)_{\left.\right|_{\alpha(t)}}+\text { mult }_{t}\left(\alpha_{0}(t)\right) \\
& =\text { mult }_{t}\left(\frac{\partial h}{\partial z_{0}}\right)_{\left.\right|_{\alpha(t)}}+\text { mult }_{t} z_{0}(\alpha(t))
\end{aligned}
$$

\subsection{Coordenadas pré-polares}

Ressaltamos que até agora não é possível garantir a existência das multiplicidades polares e dos números de Lê. Porém, se considerarmos uma escolha genérica de coordenadas, estes estão definidos. As coordenadas são escolhidas genericamente com respeito a um certo tipo de estratificação da hipersuperfície definida pela $h$.

Definição 3.26. Se X é um espaço analítico, uma estratificação analítica de X é uma partição localmente finita, $\left\{S_{\alpha}\right\}$, de X em subvariedades analíticas - os estratos - cujo fecho de cada estrato é também analítico, e tal que $\left\{S_{\alpha}\right\}$ satisfaz as condições de fronteira, isto é, o fecho de cada estrato é a união de estratos.

Definição 3.27. Uma boa estratificação de $h$ no ponto $p \in V(h)$ é uma estratificação, $\mathscr{B}$, da hipersuperfície $V(h)$ em uma vizinhança, $U$, de p tal que a parte suave de $V(h)$ é um estrato e de modo que a estratificação satisfaça $a_{h}$ condição de Thom com respeito a $U-V(h)$, ou seja, se $q_{i}$ é uma sequência de pontos em $U-V(h)$ tal que $q_{i} \rightarrow q \in S \in \mathscr{B}$ e $T_{q_{i}} V\left(h-h\left(q_{i}\right)\right)$ converge para algum hiperplano $T$, então $T_{q} S \subseteq T$.

Proposição 3.28. Existe uma boa estratificação para toda $h:(U, 0) \rightarrow(\mathbb{C}, 0)$ em todo $p \in V(h)$. 
A noção definida a seguir, coordenadas pré-polares, é crucial durante todo o trabalho. Ela fornece uma condição genérica na escolha linear das coordenadas que implica que todos as multiplicidades polares e os números de Lê estão definidos. Além disso, a prepolaridade pode ser vista como condição para obter alguns resultados topológicos.

Definição 3.29. Suponha que $\left\{S_{\alpha}\right\}$ é uma boa estratificação de hem uma vizinhança, $U$, da origem. Seja $p \in V(h)$. Então, um hiperplano, $H$, em $\mathbb{C}^{n+1}$ através de pé um pedaço pré-polar de hem $p$ com respeito a $\left\{S_{\alpha}\right\}$ se $H$ intercepta transversalmente todos os estratos de $\left\{S_{\alpha}\right\}$ exceto talvez o estrato $\{p\}$ nele mesmo - em uma vizinhança de $p$.

Se $H$ é um pedaço pré-polar de $h$ em $p$ com respeito a $\left\{S_{\alpha}\right\}$, então os germes de conjuntos em $p, \Sigma\left(h_{\mid H}\right)=(\Sigma(h)) \cap H$ e $\operatorname{dim}_{p} \Sigma\left(h_{\mid H}\right)=\left(\operatorname{dim}_{p} \Sigma(h)\right)-1$ fornece que $\operatorname{dim}_{p} \Sigma(h) \geq 1$; além disso $\left\{H \cap S_{\alpha}\right\}$ é uma boa estratificação de $h_{\left.\right|_{H}}$ em $p$ (ver [8]).

Por 2.1.3 de [8], para uma boa estratificação de $h$ fixada, os pedaços pré-polares são genéricos.

Dizemos simplesmente que $H$ é um pedaço pré-polar de $h$ em $p$ se existe uma boa estratificação tal que $H$ é pedaço pré-polar.

Sejam $\left(z_{0}, \ldots, z_{n}\right)$ uma escolha linear de coordenadas para $\mathbb{C}^{n+1}, p \in V(h)$ e $\left\{S_{\alpha}\right\}$ uma boa estratificação para $h$ em $p$.

Para $0 \leq i \leq n,\left(z_{0}, \ldots, z_{i}\right)$ é uma $(i+1)$-upla pré-polar de $h$ em $p$ com respeito a $\left\{S_{\alpha}\right\}$ se, e somente se, $V\left(z_{0}-p_{0}\right)$ é um pedaço pré-polar de $h$ em $p$ com respeito a $\left\{S_{\alpha}\right\}$ e para todo $j$ tal que $1 \leq j \leq i, V\left(z_{j}-p_{j}\right)$ é um pedaço pré-polar de $h_{\left.\right|_{V\left(z_{0}-p_{0}, \ldots, z_{j-1}-p_{j-1}\right)}}$ em $p$ com respeito a $\left\{S_{\alpha} \cap V\left(z_{0}-p_{0}, \ldots, z_{j-1}-p_{j-1}\right)\right\}$.

Como os pedaços pré-polares são genéricos, também são as $(i+1)$-uplas pré-polares.

Naturalmente, dizemos que $\left(z_{0}, \ldots, z_{i}\right)$ é uma $(i+1)$-upla pré-polar de $h$ em $p$ se existe uma boa estratificação de $h$ em $p$ tal que $\left(z_{0}, \ldots, z_{i}\right)$ é uma $(i+1)$-upla pré-polar.

Finalmente, dizemos que as coordenadas $\left(z_{0}, \ldots, z_{n}\right)$ são pré-polares de $h$ se, e somente se, para todo $p \in V(h)$, se $s$ denota $\operatorname{dim}_{p} \Sigma(h)$, então $\left(z_{0}, \ldots, z_{s-1}\right)$ é uma $s$-upla pré-polar de $h$ em $p$ (se $s=0$ ou $p \notin \Sigma(h)$, queremos dizer que não existe condição nas coordenadas). 
Mostramos que por uma escolha de coordenadas que são pré-polares é garantida a existência das multiplicidades polares e dos números de Lê.

Lema 3.30. Seja $L_{k}=\left(z_{0}, \ldots, z_{k}\right)$ pré-polar de $h$ na origem. Então, para todo $p \in V(h) \cap$ $V\left(z_{0}, \ldots, z_{k-1}\right)$ próximo de 0 , se $T_{p}$ é um hiperplano tal que exista uma sequência $p_{i} \notin \Sigma(h)$ com $p_{i} \rightarrow$ p e $T_{p_{i}} V\left(h-h\left(p_{i}\right)\right) \rightarrow T_{p}$, então $V\left(L_{k}\right)=T_{p} V\left(L_{k}-L_{k}(p)\right) \nsubseteq T_{p}$.

Demonstração. Fixe uma boa estratificação, $\mathscr{B}$, com respeito a que $L_{k}$ é prepolar. A demonstração é por indução em $k$.

Para $k=0, V\left(z_{0}, \ldots, z_{k-1}\right)$ é igual ao espaço ambiente inteiro, $\mathbb{C}^{n+1}$ e a afirmação segue da definição de pedaço pré-polar.

Suponha que o lema é verdadeiro para $k$, mas falso para $k+1$. Então, deve existir pontos $p$ arbitrariamente próximos da origem com as seguintes propriedades:

$p \in V(h) \cap V\left(z_{0}, \ldots, z_{k}\right)$ e existe uma sequência $p_{i} \notin \Sigma(h)$ com $p_{i} \rightarrow p$ e $T_{p_{i}} V\left(h-h\left(p_{i}\right)\right)$ convergindo para algum hiperplano $T_{p} \operatorname{com} V\left(z_{0}, \ldots, z_{k+1}\right) \subseteq T_{p}$.

Tomando uma subsequência, podemos assumir que os $p_{i}^{\prime} s$ estão contidos em um único bom estrato, $G$. Pela hipótese indutiva, também podemos assumir que $V\left(z_{0}, \ldots, z_{k}\right) \nsubseteq T_{p}$.

Assim,

$\operatorname{dim}\left(T_{p} \cap V\left(z_{0}, \ldots, z_{k}\right)\right)=n-k-1=\operatorname{dim}\left(T_{p} \cap V\left(z_{0}, \ldots, z_{k+1}\right)\right)$ e $T_{p} \cap V\left(z_{0}, \ldots, z_{k}\right) \subseteq V\left(z_{k+1}\right)$.

Por isso, $T_{p}\left(G \cap V\left(z_{0}, \ldots, z_{k}\right)\right)=T_{p}(G) \cap V\left(z_{0}, \ldots, z_{k}\right) \subseteq T_{p} \cap V\left(z_{0}, \ldots, z_{k}\right) \subseteq V\left(z_{k+1}\right)$, que é uma contradição, pois $V\left(z_{k+1}\right)$ é um pedaço pré-polar de $h_{\left.\right|_{V\left(z_{0}, \ldots, z_{k}\right)}}$ em 0 com respeito a boa estratificação $\mathscr{B} \cap V\left(z_{0}, \ldots, z_{k}\right)$.

Teorema 3.31. Sejam $p \in V(h)$ e $s=\operatorname{dim}_{p} \Sigma(h)$. Então, para uma escolha genérica de coordenadas, todos os números de Lê e as multiplicidades polares de hem p em dimensões menores ou iguais a s estão definidos. Mais precisamente, se $\left(z_{0}, \ldots, z_{k}\right)$ é pré-polar de h em p então, para todo i com $0 \leq i \leq k, \lambda_{h, z}^{i}(p)$ e $\gamma_{h, z}^{i+1}(p)$ existem. Além disso, se $\left(z_{0}, \ldots, z_{s-1}\right)$ é pré-polar de $h$ em p então, para todo $i$ com $0 \leq i \leq s, \lambda_{h, z}^{i}(p)$ e $\gamma_{h, z}^{i}(p)$ existem. 
Demonstração. Note que, como pedaços pré-polares interceptam transversalmente o estrato suave, pelo Corolário 3.24 temos que demonstrar somente que: se $\left(z_{0}, \ldots, z_{k}\right)$ é pré-polar de $h$ em $p$ então, para todo $i \operatorname{com} 0 \leq i \leq k, \lambda_{h, z}^{i}(p)$ e $\gamma_{h, z}^{i}(p)$ existem. Logo, a existência de $\gamma_{h, z}^{i+1}(p)$ segue. É suficiente demonstrar isso para $p=0$.

Se $\left(z_{0}, \ldots, z_{k}\right)$ é pré-polar, então segue da definição que $\left(z_{0}, \ldots, z_{i}\right)$ é pré-polar para todo $i \leq k$. Daí, é suficiente demonstrar que se $\left(z_{0}, \ldots, z_{k}\right)$ é pré-polar, então $\lambda_{h, z}^{k}(0)$ e $\gamma_{h, z}^{k}(0)$ existem.

Sejam $L_{k-1}=\left(z_{0}, \ldots, z_{k-1}\right)$ e $L_{k}=\left(z_{0}, \ldots, z_{k}\right)$. Seja $\mathscr{B}$ uma boa estratificação de $h$ na origem tal que $L_{k}$ é pré-polar.

Desejamos mostrar que $\Gamma_{h, L_{k}}^{k+1} \cap V\left(\frac{\partial h}{\partial z_{k}}\right) \cap V\left(L_{k-1}\right)$ é 0 -dimensional na origem.

Notemos que é suficiente mostrar que $\Gamma_{h, L_{k}}^{k+1} \cap V(h) \cap V\left(L_{k-1}\right)$ é 0-dimensional na origem. Suponha que exista uma curva analítica $\alpha$ passando pela origem em $\Gamma_{h, L_{k}}^{k+1} \cap V\left(\frac{\partial h}{\partial z_{k}}\right) \cap V\left(L_{k-1}\right)$. Seja $c(t)=h(\alpha(t))$.

Temos que

$$
\frac{\partial c}{\partial t}(t)=\frac{\partial h}{\partial z_{0}}(\alpha(t)) \alpha_{0}^{\prime}(t)+\ldots+\frac{\partial h}{\partial z_{n}}(\alpha(t)) \alpha_{n}^{\prime}(t)
$$

que é igual a 0 pois $\alpha(t) \in \Gamma_{h, L_{k}}^{k+1} \cap V\left(\frac{\partial h}{\partial z_{k}}\right) \cap V\left(L_{k-1}\right)$.

Daí, c(t) é constante e como $c(0)=0, c \equiv 0$. Isso implica que $\alpha \in \Gamma_{h, L_{k}}^{k+1} \cap V(h) \cap V\left(L_{k-1}\right)$. Agora, isso contradiz com o fato de $\Gamma_{h, L_{k}}^{k+1} \cap V(h) \cap V\left(L_{k-1}\right)$ ser 0-dimensional.

Suponha que temos uma sequência de $p$ em $\Gamma_{h, L_{k}}^{k+1} \cap V(h) \cap V\left(L_{k-1}\right)$ aproximando-se de 0 . Para cada $p$ é possível considerar uma sequência $p_{i}$ em $\Gamma_{h, L_{k}}^{k+1} \cap V(h) \cap V\left(L_{k-1}\right)$ com $p_{i} \rightarrow p$ tal que $p_{i} \notin \Sigma(h)$ e $V\left(L_{k}-L_{k}\left(p_{i}\right)\right)=T_{p_{i}} V\left(L_{k}-L_{k}\left(p_{i}\right)\right) \subseteq T_{p_{i}} V\left(h-h\left(p_{i}\right)\right)$ pois $T_{p_{i}} V\left(h-h\left(p_{i}\right)\right)$ é da forma

$$
\left\{\left(z_{0}, \ldots, z_{n}\right) \mid\left(\frac{\partial h}{\partial z_{0}}\right)\left(p_{i}\right)\left(z_{0}-\left(p_{i}\right)_{0}\right)+\ldots+\left(\frac{\partial h}{\partial z_{n}}\right)\left(p_{i}\right)\left(z_{n}-\left(p_{i}\right)_{n}\right)=0\right\}
$$

Tomando uma subsequência dos $p_{i}^{\prime}$ s, podemos assumir que $T_{p_{i}} V\left(h-h\left(p_{i}\right)\right)$ converge para algum hiperplano, $T_{p}$. Mas, por construção, $V\left(L_{k}\right)=T_{p} V\left(L_{k}-L_{k}(p)\right) \subseteq T_{p}$, que contradiz o lema. 
Falta mostrar que se $\left(z_{0}, \ldots, z_{s-1}\right)$ é pré-polar de $h$ em 0 , então $\lambda_{h, z}^{s}(0)$ existe; em outras palavras obtemos a última dimensão sem afirmação extra.

Independente da escolha de $z_{s}, \Gamma_{h, L_{s}}^{s+1}=V\left(\frac{\partial h}{\partial z_{s+1}}, \ldots, \frac{\partial h}{\partial z_{n}}\right)$ e assim, como conjuntos,

$$
\Gamma_{h, L_{s}}^{s+1} \cap V\left(\frac{\partial h}{\partial z_{s}}\right) \cap V\left(z_{0}, \ldots, z_{s-1}\right)=\Sigma\left(h_{\left.\left.\right|_{V\left(z_{0}, \ldots, z_{s-1}\right)}\right)}\right) .
$$

Agora, $\Sigma\left(h_{\left.\left.\right|_{V\left(z_{0}, \ldots, z_{s-1}\right)}\right)}\right)$ é 0-dimensional na origem desde que cada pedaço sucessível seja prépolar.

Observação 3.32. Enquanto é verdadeiro que coordenadas pré-polares ocorrem genericamente e garantem a existência dos números de Lê, não é verdadeiro que todos os conjuntos de coordenadas pré-polares produzem o mesmo número de Lê.

Se $\operatorname{dim}_{p} \Sigma(h)=1$, então $\{V(h)-\Sigma(h), \Sigma(h)-p, p\}$ é uma boa estratificação de $h$ em uma vizinhança de $p$; daí, $V\left(z_{0}-p_{0}\right)$ é um pedaço pré-polar se, e somente se, $\operatorname{dim}_{p} \Sigma\left(h_{\left.\right|_{V\left(z_{0}-p_{0}\right)}}\right)=0$. Neste caso se, e somente se, $\gamma_{h, z}^{1}(p)$ e $\lambda_{h, z}^{1}(p)$ estão definidos.

Exemplo 3.33. Seja $h=x y z$ e assim $V(h)$ consiste dos planos coordenados em $\mathbb{C}^{3}$. (Ver exemplo 2.7). Então, $\Sigma(h)=V(x, y) \cup V(x, z) \cup V(y, z)$, que é a união dos três eixos coordenados.

As coordenadas $(x, y, z)$ são extremamente não genéricas pois estas são assintóticas ao conjunto singular. Assim escolhemos as coordenadas genéricas $\tilde{z}=\left(\tilde{z}_{0}, \tilde{z}_{1}, \tilde{z}_{2}\right)$. Então, o conjunto $\left|\Lambda_{h, \tilde{z}}^{1}\right|=\Sigma(h)$. Consequentemente,

$$
\lambda_{h, \tilde{z}}^{1}(0)=\left(\Lambda_{h, \tilde{z}}^{1} \cdot V\left(\tilde{z}_{0}\right)\right)_{0}=\sum_{p}\left(\Lambda_{h, \tilde{z}}^{1} \cdot V\left(\tilde{z}_{0}-\xi\right)\right)_{p}=\sum_{p} \lambda_{h, \tilde{z}}^{1}(p)
$$

onde a soma é para todo $p \in \stackrel{\circ}{B_{\varepsilon}} \cap \Lambda_{h, \tilde{z}}^{1} \cap V\left(\tilde{z}_{0}-\xi\right)$ para $\varepsilon$ pequeno e $0<\xi \leq \varepsilon$; esse conjunto consiste de três pontos e, por simetria, $\lambda^{1}$ deve ser o mesmo em cada um desses três pontos.

Desejamos usar a Proposição 3.23 para calcular $\lambda_{h, \tilde{z}}^{1}(p)$.

Como cada $p \in \Sigma(h)$, segue de 3.12 que $\gamma_{h, \tilde{z}}^{1}$ está contido somente em $\Lambda_{h, \tilde{z}}^{0}$, que é genericamente 0-dimensional. Assim, nossos pontos $p$ são tais que $\gamma_{h, \tilde{z}}^{1}(p)=0$, e segue de 3.23 que 
$\lambda_{h, \tilde{z}}^{1}(p)=\lambda_{h_{V\left(\tilde{z}_{0}-p_{0}\right)}^{0}}^{0}(p)$, onde $\widehat{z}$ denota a restrição das coordenadas $\widetilde{z}$ por $V\left(\tilde{z}_{0}-p_{0}\right)$.

Agora, $h_{\left.\right|_{V\left(\tilde{z}_{0}-p_{0}\right)}}$ tem uma singularidade isolada em cada um dos nossos três pontos $p$, e $\operatorname{assim} \lambda_{h_{\left.\right|_{V\left(\tilde{z}_{0}-p_{0}\right)}}^{0}, \widehat{z}}(p)=$ número de Milnor de $h_{\left.\right|_{V\left(\tilde{z}_{0}-p_{0}\right)}}$ em $p$, e esse é visto ser igual a 1. Segue, finalmente, que $\lambda_{h, \tilde{z}}^{1}(0)=3$.

O valor genérico de $\lambda_{h, \tilde{z}}^{0}(0)$ é um tanto complicado para calcular e será mostrado como um caso mais geral no exemplo 3.35. Contudo, afirmamos que $\lambda_{h, \tilde{z}}^{0}(0)=2$.

Observação 3.34. Seja $U$ um subconjunto aberto de $\mathbb{C}^{n+1}$, seja $z=\left(z_{0}, \ldots, z_{n}\right)$ as coordenadas para $\mathbb{C}^{n+1}$ e $h: U \rightarrow \mathbb{C}$ uma função analítica qualquer. As coordenadas $z$ podem não ser genéricas para $h$.

Desejamos ver como calcular $\lambda_{h, \tilde{z}}^{0}$ para uma escolha linear genérica de $\tilde{z}$.

Assim, seja $\tilde{z}$ uma escolha linear genérica de coordenadas para $\mathbb{C}^{n+1}$ e seja $a_{i j}=\frac{\partial z_{i}}{\partial \tilde{z}_{j}}$.

Agora,

$$
\begin{aligned}
\Gamma_{h, \tilde{z}}^{1} & =V\left(\frac{\partial h}{\partial \tilde{z}_{1}}, \ldots, \frac{\partial h}{\partial \tilde{z}_{n}}\right) / \Sigma(h) \\
& =V\left(a_{01} \frac{\partial h}{\partial z_{0}}+\ldots+a_{n 1} \frac{\partial h}{\partial z_{n}}, \ldots, a_{0 n} \frac{\partial h}{\partial z_{0}}+\ldots+a_{n n} \frac{\partial h}{\partial z_{n}}\right) / \Sigma(h) .
\end{aligned}
$$

Pela realização de sucessivas operações elementares, achamos que o ideal

$$
\left\langle a_{01} \frac{\partial h}{\partial z_{0}}+\ldots+a_{n 1} \frac{\partial h}{\partial z_{n}}, \ldots, a_{0 n} \frac{\partial h}{\partial z_{0}}+\ldots+a_{n n} \frac{\partial h}{\partial z_{n}}\right\rangle
$$

é gerado por

$$
\frac{\partial h}{\partial z_{0}}+b_{0} \frac{\partial h}{\partial z_{n}}, \frac{\partial h}{\partial z_{1}}+b_{1} \frac{\partial h}{\partial z_{n}}, \ldots, \frac{\partial h}{\partial z_{n-1}}+b_{n-1} \frac{\partial h}{\partial z_{n}}
$$

onde $b_{0}, \ldots, b_{n-1}$ são genéricos $\neq 0$.

Assim,

$$
\Gamma_{h, \tilde{z}}^{1}=V\left(\frac{\partial h}{\partial z_{0}}+b_{0} \frac{\partial h}{\partial z_{n}}, \frac{\partial h}{\partial z_{1}}+b_{1} \frac{\partial h}{\partial z_{n}}, \ldots, \frac{\partial h}{\partial z_{n-1}}+b_{n-1} \frac{\partial h}{\partial z_{n}}\right) / \Sigma(h),
$$


e $\Lambda_{h, z}^{0}$ é dado pela intersecção desses com $a_{00} \frac{\partial h}{\partial z_{0}}+\ldots+a_{n 0} \frac{\partial h}{\partial z_{n}}$.

Exemplo 3.35. Podemos usar a observação anterior para calcular o valor genérico de $\lambda^{0}$ no exemplo 3.33. Na verdade, podemos fazer um cálculo mais geral.

Seja $h=z_{0} z_{1} \ldots z_{n}$, assim $V(h)$ é a união dos planos coordenados em $\mathbb{C}^{n+1}$ e $\Sigma(h)$ a união de intersecções de pares dos diferentes planos coordenados. Desejamos mostrar, por uma escolha genérica de coordenadas, $\tilde{z}$, que $\lambda_{h, \tilde{z}}^{0}(0)=n$.

Pela observação anterior, encontramos que $\Gamma_{h, z}^{1}$ é igual a

$$
V\left(z_{1} z_{2} \ldots z_{n-1}\left(z_{n}+b_{0} z_{0}\right), z_{0} z_{2} \ldots z_{n-1}\left(z_{n}+b_{1} z_{1}\right), \ldots, z_{0} z_{1} \ldots z_{n-2}\left(z_{n}+b_{n-1} z_{n-1}\right)\right) / \Sigma(h)
$$

Aplicando 3.4 iii repetitivamente, concluímos que

$$
\Gamma_{h, z}^{1}=V\left(z_{n}+b_{0} z_{0}, z_{n}+b_{1} z_{1}, \ldots, z_{n}+b_{n-1} z_{n-1}\right)
$$

Finalmente, pela intersecção desse com

$$
V\left(\frac{\partial h}{\partial \tilde{z}_{0}}\right)=V\left(a_{00} z_{1} z_{2} \ldots z_{n}+\ldots+a_{n 0} z_{0} z_{1} \ldots z_{n-1}\right)
$$

obtemos o resultado desejado $\lambda_{h, \tilde{z}}^{0}(0)=n$.

Observamos aqui que se o sistema de coordenadas é suficientemente genérico, então $\lambda_{h, z}^{s}(p)$ é simplesmente a multiplicidade do jacobiano de $h$, considerado como o espaço analítico complexo definido pelo anulamento do ideal jacobiano em $p$. (Ver [14] página 21). 


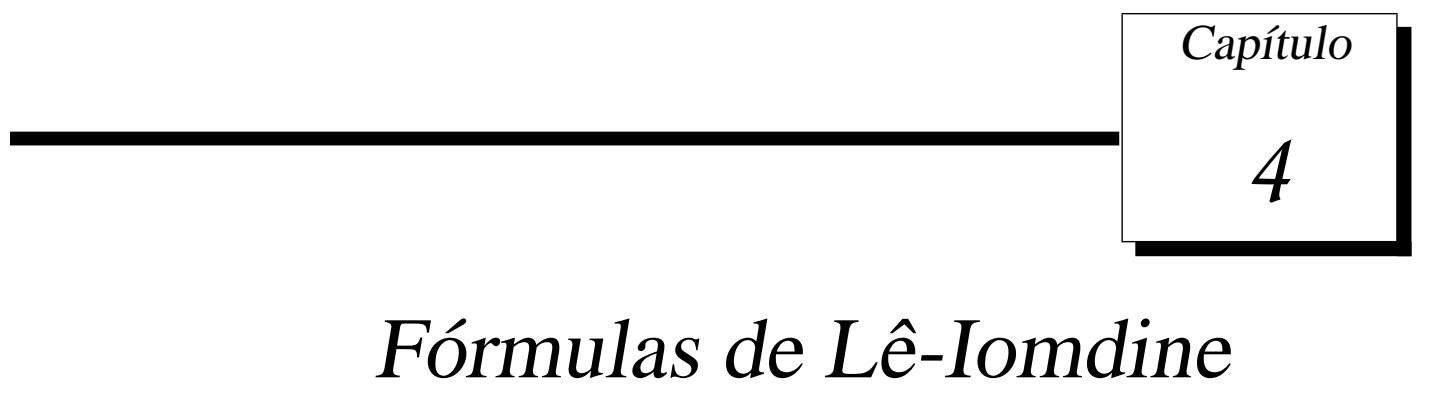

Neste capítulo, as fórmulas de Lê-Iomdine são descritas para funções com conjunto crítico de dimensão arbitrária. Essas fórmulas descrevem como os números de Lê de uma hipersuperfície singular estão relacionados com os números de Lê de uma certa sequência de hipersuperfícies singulares $X_{0}, \ldots, X_{s-1}$ que se aproxima da singularidade original de tal forma que os conjuntos críticos de seus termos $X_{i}$ têm dimensão $i$.

O raio polar é um número associado à curva polar que garante a existência desta sequência de hipersuperfícies.

Definição 4.1. Suponha que $\Gamma_{h, z_{0}}^{1}$ é 1-dimensional na origem. Seja $\eta$ uma componente irredutível de $\Gamma_{h, z_{0}}^{1}$ (com sua estrutura reduzida). Se $\eta \cap V\left(z_{0}\right)$ é 0-dimensional na origem, definimos o raio polar de $\eta$ (de h em 0 com respeito a $z_{0}$ ) como sendo

$$
\frac{(\eta \cdot V(h))_{0}}{\left(\eta \cdot V\left(z_{0}\right)\right)_{0}}
$$

Se $\eta \cap V\left(z_{0}\right)$ não é 0-dimensional na origem $\left(\eta \subseteq V\left(z_{0}\right)\right)$ então o raio polar de $\eta$ é 1 .

Um raio polar (de $h$ em 0 com respeito a $z_{0}$ ) é o raio polar de qualquer componente da curva polar.

Observação 4.2. Temos que

$$
\frac{(\eta \cdot V(h))_{0}}{\left(\eta \cdot V\left(z_{0}\right)\right)_{0}}=\frac{\left(\eta \cdot V\left(\frac{\partial h}{\partial z_{0}}\right)\right)_{0}+\left(\eta \cdot V\left(z_{0}\right)\right)_{0}}{\left(\eta \cdot V\left(z_{0}\right)\right)_{0}}=\frac{\left(\eta \cdot V\left(\frac{\partial h}{\partial z_{0}}\right)\right)_{0}}{\left(\eta \cdot V\left(z_{0}\right)\right)_{0}}+1 .
$$


Observação 4.3. Notemos que no caso em que $h$ é um polinômio homogêneo de grau d e que $\Gamma_{h, z_{0}}^{1}$ é 1- dimensional na origem, as componentes irredutíveis da curva polar são retas e os possíveis raios polares são 1 e $d$.

Nosso próximo resultado será obter as fórmulas de Lê-Iomdine para uma hipersuperfície com conjunto singular qualquer; essas fórmulas são ferramentas muito úteis para questões de redução de hipersuperfícies singulares gerais para casos mais fáceis de hipersuperfícies com singularidades isoladas. As fórmulas dizem como os números de Lê de $h$ mudam quando uma grande potência de uma variável é adicionada.

O próximo lema é o primeiro passo para a obtenção destas fórmulas.

Lema 4.4. Sejam $h:(U, 0) \rightarrow(\mathbb{C}, 0)$ uma função analítica com $U \subseteq \mathbb{C}^{n+1}$ aberto e $s=\operatorname{dim}_{0} \Sigma(h)$ com $s \geq 1$. Considere $z=\left(z_{0}, \ldots, z_{n}\right)$ uma escolha linear de coordenadas tais que $\lambda_{h, z}^{i}(0)$ está definido para todo $i \leq s$. Seja a um número complexo não nulo e use as coordenadas $\widetilde{z}=\left(z_{1}, \ldots, z_{n}, z_{0}\right)$ para $h+a z_{0}^{j}$, onde $j \geq 2$.

Se j é maior ou igual ao raio polar máximo de hentão, para todos exceto um número finito de complexos $a$,

i) $\operatorname{dim}_{0}\left(\Gamma_{h, z}^{1} \cap V\left(\frac{\partial h}{\partial z_{0}}+j a z_{0}^{j-1}\right)\right)=0$;

ii) $\lambda_{h, z}^{0}(0)=\left(\Gamma_{h, z}^{1} \cdot V\left(\frac{\partial h}{\partial z_{0}}+j a z_{0}^{j-1}\right)\right)_{0}$;

iii) $\Sigma\left(h+a z_{0}^{j}\right)$ é $(s-1)$ dimensional na origem e é igual a $\Sigma(h) \cap V\left(z_{0}\right)$ como germes de conjuntos em 0 ;

iv) Se $i \geq 1$ então temos uma igualdade de ciclos $\Gamma_{h+a z_{0}^{j}, \tilde{z}}^{i}=\Gamma_{h, z}^{i+1} \cdot V\left(\frac{\partial h}{\partial z_{0}}+j a z_{0}^{j-1}\right)$ próximo da origem;

v) $\left(\Gamma_{h+a w^{j}, w-z_{0}}^{1} \cdot V\left(h+a w^{j}\right)\right)_{0}=j \lambda_{h, z}^{0}(0)$ onde w é uma variável disjunta das de $h$.

Além disso, se j é estritamente maior que raio polar máximo de $h$, as igualdades acima mantem-se para todo a não nulo. Em particular, esse é o caso se $j \geq 2+\lambda_{h, z}^{0}(0)$. 


\section{Demonstração.}

i) e ii) Como $\lambda_{h, z}^{0}(0)$ está definido, $\Gamma_{h, z}^{1}$ é 1-dimensional em 0. (Proposição 3.25)

Escrevendo $\Gamma_{h, z}^{1}=\sum_{\eta} k_{\eta}[\eta]$, onde $\eta$ são as componentes irredutíveis de $\Gamma_{h, z}^{1}$, temos

$$
\left(\Gamma_{h, z}^{1} \cdot V\left(\frac{\partial h}{\partial z_{0}}+j a z_{0}^{j-1}\right)\right)_{0}=\sum_{\eta} k_{\eta}\left(\eta \cdot V\left(\frac{\partial h}{\partial z_{0}}+j a z_{0}^{j-1}\right)\right)_{0} .
$$

Seja $\alpha_{\eta}(t)$ uma parametrização de $\eta$. Assim,

$$
\left(\eta \cdot V\left(\frac{\partial h}{\partial z_{0}}+j a z_{0}^{j-1}\right)\right)_{0}=\operatorname{mult}_{t}\left(\left(\frac{\partial h}{\partial z_{0}}+j a z_{0}^{j-1}\right)\left(\alpha_{\eta}(t)\right)\right)
$$

e com exceção, possivelmente, do único valor de $a$ que faz com que os termos de menor grau de $\frac{\partial h}{\partial z_{0}}\left(\alpha_{\eta}(t)\right)$ e $j a z_{0}^{j-1}\left(\alpha_{\eta}(t)\right)$ se anulem,

$$
\begin{aligned}
& =\text { mínimo }\left\{\operatorname{mult}_{t}\left(\frac{\partial h}{\partial z_{0}}\left(\alpha_{\eta}(t)\right)\right), \text { mult }_{t}\left(j a z_{0}^{j-1}\left(\alpha_{\eta}(t)\right)\right)\right\} \\
& =\text { mínimo }\left\{\left(\eta \cdot V\left(\frac{\partial h}{\partial z_{0}}\right)\right)_{0},\left(\eta \cdot V\left(j a z_{0}^{j-1}\right)\right)_{0}\right\} \\
& =\text { mínimo }\left\{\left(\eta \cdot V\left(\frac{\partial h}{\partial z_{0}}\right)\right)_{0},\left(\eta \cdot V\left(z_{0}^{j-1}\right)\right)_{0}\right\} .
\end{aligned}
$$

Por outro lado, usando que $\left(\eta \cdot V\left(z_{0}^{j-1}\right)\right)_{0}=(j-1)\left(\eta \cdot V\left(z_{0}\right)\right)_{0}$, e como por hipótese

$$
j \geq \frac{\left(\eta \cdot V\left(\frac{\partial h}{\partial z_{0}}\right)\right)_{0}}{\left(\eta \cdot V\left(z_{0}\right)\right)_{0}}+1
$$

$\operatorname{temos}\left(\eta \cdot V\left(z_{0}^{j-1}\right)\right)_{0} \geq\left(\eta \cdot V\left(\frac{\partial h}{\partial z_{0}}\right)\right)_{0}$

Assim, $\left(\eta \cdot V\left(\frac{\partial h}{\partial z_{0}}+j a z_{0}^{j-1}\right)\right)_{0}=\left(\eta \cdot V\left(\frac{\partial h}{\partial z_{0}}\right)\right)_{0}$ e concluímos que

$$
\left(\Gamma_{h, z}^{1} \cdot V\left(\frac{\partial h}{\partial z_{0}}+j a z_{0}^{j-1}\right)\right)_{0}=\left(\Gamma_{h, z}^{1} \cdot V\left(\frac{\partial h}{\partial z_{0}}\right)\right)_{0}=\lambda_{h, z}^{0}(0) .
$$


iii) Como conjuntos,

$$
\begin{aligned}
\Sigma\left(h+a z_{0}^{j}\right) & =V\left(\frac{\partial h}{\partial z_{0}}+j a z_{0}^{j-1}, \frac{\partial h}{\partial z_{1}}, \ldots, \frac{\partial h}{\partial z_{n}}\right)=V\left(\frac{\partial h}{\partial z_{0}}+j a z_{0}^{j-1}\right) \cap V\left(\frac{\partial h}{\partial z_{1}}, \ldots, \frac{\partial h}{\partial z_{n}}\right) \\
& =V\left(\frac{\partial h}{\partial z_{0}}+j a z_{0}^{j-1}\right) \cap\left(\Gamma_{h, z}^{1} \cup \Sigma(h)\right) \\
& =\left(V\left(\frac{\partial h}{\partial z_{0}}+j a z_{0}^{j-1}\right) \cap \Gamma_{h, z}^{1}\right) \cup\left(V\left(\frac{\partial h}{\partial z_{0}}+j a z_{0}^{j-1}\right) \cap \Sigma(h)\right) \\
& =\left(V\left(\frac{\partial h}{\partial z_{0}}+j a z_{0}^{j-1}\right) \cap \Gamma_{h, z}^{1}\right) \cup\left(V\left(z_{0}\right) \cap \Sigma(h)\right) .
\end{aligned}
$$

Pelo item i) $V\left(\frac{\partial h}{\partial z_{0}}+j a z_{0}^{j-1}\right) \cap \Gamma_{h, z}^{1}$ é 0-dimensional. Assim, próximo da origem, $\Sigma\left(h+a z_{0}^{j}\right)=V\left(z_{0}\right) \cap \Sigma(h)$ e pela Proposição 3.17 tem dimensão $s-1$.

iv) Usando o item $\mathrm{iii}$ ) temos

$$
\begin{aligned}
\Gamma_{h+a z_{0}^{j}, \tilde{z}}^{i} & =V\left(\frac{\partial h}{\partial z_{i+1}}, \ldots, \frac{\partial h}{\partial z_{n}}, \frac{\partial h}{\partial z_{0}}+j a z_{0}^{j-1}\right) / \Sigma\left(h+a z_{0}^{j}\right) \\
& \left.=V\left(\frac{\partial h}{\partial z_{i+1}}, \ldots, \frac{\partial h}{\partial z_{n}}\right) \cap V\left(\frac{\partial h}{\partial z_{0}}+j a z_{0}^{j-1}\right) / \Sigma(h) \cap V\left(z_{0}\right)\right) \\
& =\left(\Gamma_{h, z}^{i+1} \cup R\right) \cap V\left(\frac{\partial h}{\partial z_{0}}+j a z_{0}^{j-1}\right) /\left(\Sigma(h) \cap V\left(z_{0}\right)\right),
\end{aligned}
$$

onde o ideal definindo o espaço analítico complexo $R$ consiste das intersecções das componentes primárias, $q$, de qualquer decomposição primária do ideal $\left\langle\frac{\partial h}{\partial z_{i+1}}, \ldots, \frac{\partial h}{\partial z_{n}}\right\rangle$ tal que $|V(q)| \subseteq$ $|\Sigma(h)|$.

Independente da decomposição primária $|R| \subseteq|\Sigma(h)|$ e assim $\left|R \cap V\left(\frac{\partial h}{\partial z_{0}}+j a z_{0}^{j-1}\right)\right| \subseteq$ $\left|\Sigma(h) \cap V\left(z_{0}\right)\right|$. 
Aplicando 3.4 ii temos

$$
\begin{aligned}
\Gamma_{h+a z_{0}^{j}, \tilde{z}}^{i} & =\left(\Gamma_{h, z}^{i+1} \cup R\right) \cap V\left(\frac{\partial h}{\partial z_{0}}+j a z_{0}^{j-1}\right) /\left(\Sigma(h) \cap V\left(z_{0}\right)\right) \\
& =\left(\Gamma_{h, z}^{i+1} \cap V\left(\frac{\partial h}{\partial z_{0}}+j a z_{0}^{j-1}\right)\right) /\left(\Sigma(h) \cap V\left(z_{0}\right)\right) .
\end{aligned}
$$

Pelo número de equações, a dimensão de toda componente de $\Gamma_{h, z}^{i+1} \cap V\left(\frac{\partial h}{\partial z_{0}}+j a z_{0}^{j-1}\right)$ é no mínimo $i$.

Agora, $\Gamma_{h, z}^{i+1} \cap V\left(\frac{\partial h}{\partial z_{0}}+j a z_{0}^{j-1}\right) \cap \Sigma(h) \cap V\left(z_{0}\right)=\Gamma_{h, z}^{i+1} \cap \Sigma(h) \cap V\left(z_{0}\right)=\bigcup_{k \leq i} \Lambda_{h, z}^{k} \cap V\left(z_{0}\right)$.

Como todos os números de Lê estão definidos na origem e $i \geq 1$, a dimensão de $\bigcup_{k \leq i} \Lambda_{h, z}^{k} \cap V\left(z_{0}\right)$ é no máximo $i-1$.

Assim $\Gamma_{h, z}^{i+1} \cap V\left(\frac{\partial h}{\partial z_{0}}+j a z_{0}^{j-1}\right)$ não tem componente contida em $\Sigma(h) \cap V\left(z_{0}\right)$ e daí concluímos que $\Gamma_{h+a z_{0}^{j}, \tilde{z}}^{i}=\Gamma_{h, z}^{i+1} \cdot V\left(\frac{\partial h}{\partial z_{0}}+j a z_{0}^{j-1}\right)$.

v) Seja $L=w-z_{0}$ e use $\left(L, z_{0}, \ldots, z_{n}\right)$ como coordenadas para $\mathbb{C}^{n+2}$. Então $\Gamma_{h+a w^{j}, w-z_{0}}^{1}=\Gamma_{h+a\left(L+z_{0}\right)^{j}, L}^{1}=V\left(\frac{\partial h}{\partial z_{0}}+j a\left(L+z_{0}\right)^{j-1}, \frac{\partial h}{\partial z_{1}}, \ldots, \frac{\partial h}{\partial z_{n}}\right) / \Sigma\left(h+a\left(L+z_{0}\right)^{j}\right)$

que, com as coordenadas $\left(w, z_{0}, \ldots, z_{n}\right)$,

$$
=V\left(\frac{\partial h}{\partial z_{0}}+j a w^{j-1}, \frac{\partial h}{\partial z_{1}}, \ldots, \frac{\partial h}{\partial z_{n}}\right) / \Sigma\left(h+a w^{j}\right)
$$

Temos que

$$
\Sigma\left(h+a w^{j}\right)=V\left(j a w^{j-1}, \frac{\partial h}{\partial z_{0}}, \ldots, \frac{\partial h}{\partial z_{n}}\right)=\left\{\left(0, z_{0}, \ldots, z_{n}\right) \mid \frac{\partial h}{\partial z_{0}}=\ldots=\frac{\partial h}{\partial z_{n}}=0\right\}=0 \times \Sigma(h) .
$$


Daí, como espaços analíticos complexos,

$$
\begin{aligned}
\Gamma_{h+a w^{j}, w-z_{0}}^{1} & =\left(V\left(\frac{\partial h}{\partial z_{0}}+j a w^{j-1}\right) \cap V\left(\frac{\partial h}{\partial z_{1}}, \ldots, \frac{\partial h}{\partial z_{n}}\right)\right) / 0 \times \Sigma(h) \\
& =\left(V\left(\frac{\partial h}{\partial z_{0}}+j a w^{j-1}\right) \cap\left(\mathbb{C} \times\left(\Gamma_{h, z}^{1} \cup R\right)\right)\right) / 0 \times \Sigma(h) .
\end{aligned}
$$

Temos que $|R| \subseteq|\Sigma(h)|$ e assim $\left|(\mathbb{C} \times R) \cap V\left(\frac{\partial h}{\partial z_{0}}+j a w^{j-1}\right)\right| \subseteq|0 \times \Sigma(h)|$.

Aplicando 3.4 ii concluímos que

$$
\Gamma_{h+a w^{j}, w-z_{0}}^{1}=\left(V\left(\frac{\partial h}{\partial z_{0}}+j a w^{j-1}\right) \cap\left(\mathbb{C} \times \Gamma_{h, z}^{1}\right)\right) / 0 \times \Sigma(h) .
$$

Como $\operatorname{dim}_{0} V\left(\frac{\partial h}{\partial z_{0}}+j a w^{j-1}\right)=n+1$ e $\operatorname{dim}_{0}\left(\mathbb{C} \times \Gamma_{h, z}^{1}\right)=2$ (pois $\Gamma_{h, z}^{1}$ é 1-dimensional $)$, então $\operatorname{dim}_{0}\left(V\left(\frac{\partial h}{\partial z_{0}}+j a w^{j-1}\right) \cap\left(\mathbb{C} \times \Gamma_{h, z}^{1}\right)\right)$ é no mínimo 1 .

Agora,

$$
\operatorname{dim}_{0}\left(V\left(\frac{\partial h}{\partial z_{0}}+j a w^{j-1}\right) \cap\left(\mathbb{C} \times \Gamma_{h, z}^{1}\right) \cap(0 \times \Sigma(h))\right)=\operatorname{dim}_{0}\left(0 \times\left(V\left(\frac{\partial h}{\partial z_{0}}\right) \cap \Gamma_{h, z}^{1} \cap \Sigma(h)\right)\right)=0
$$

Daí, $\left(V\left(\frac{\partial h}{\partial z_{0}}+j a w^{j-1}\right) \cap\left(\mathbb{C} \times \Gamma_{h, z}^{1}\right)\right)$ não tem componente contida em $0 \times \Sigma(h)$.

Portanto como 1-ciclo na origem

$$
\Gamma_{h+a w^{j}, w-z_{0}}^{1}=V\left(\frac{\partial h}{\partial z_{0}}+j a w^{j-1}\right) \cdot\left(\mathbb{C} \times \Gamma_{h, z}^{1}\right) .
$$

Usando ambas coordenadas $\left(w, z_{0}, \ldots, z_{n}\right)$ e $\left(L, z_{0}, \ldots, z_{n}\right)$ temos:

$$
\left(\Gamma_{h+a w^{j}, w-z_{0}}^{1} \cdot V\left(h+a w^{j}\right)\right)_{0}=\left(\Gamma_{h+a\left(L+z_{0}\right)^{j}, L}^{1} \cdot V\left(h+a\left(L+z_{0}\right)^{j}\right)\right)_{0}
$$


e, aplicando a Proposição 3.25,

$$
\begin{aligned}
& =\left(\Gamma_{h+a w^{j}, w-z_{0}}^{1} \cdot V(L)\right)_{0}+\left(\Gamma_{h+a w^{j}, w-z_{0}}^{1} \cdot V\left(\frac{\partial\left(h+a\left(L+z_{0}\right)^{j}\right)}{\partial L}\right)\right)_{0} \\
& =\left(V\left(\frac{\partial h}{\partial z_{0}}+j a w^{j-1}\right) \cdot\left(\mathbb{C} \times \Gamma_{h, z}^{1}\right) \cdot V\left(w-z_{0}\right)\right)_{0} \\
& +\left(V\left(\frac{\partial h}{\partial z_{0}}+j a w^{j-1}\right) \cdot\left(\mathbb{C} \times \Gamma_{h, z}^{1}\right) \cdot V\left(j a w^{j-1}\right)\right)_{0} \\
& =\left(\left(\mathbb{C} \times V\left(\frac{\partial h}{\partial z_{0}}+j a z_{0}^{j-1}\right)\right) \cdot\left(\mathbb{C} \times \Gamma_{h, z}^{1}\right) \cdot V\left(w-z_{0}\right)\right)_{0} \\
& +(j-1)\left(V\left(\frac{\partial h}{\partial z_{0}}+j a w^{j-1}\right) \cdot\left(\mathbb{C} \times \Gamma_{h, z}^{1}\right) \cdot V(w)\right)_{0} \\
& =\left(\left(\mathbb{C} \times\left(V\left(\frac{\partial h}{\partial z_{0}}+j a z_{0}^{j-1}\right) \cdot \Gamma_{h, z}^{1}\right)\right) \cdot V\left(w-z_{0}\right)\right)_{0}+(j-1)\left(0 \times\left(\Gamma_{h, z}^{1} \cdot V\left(\frac{\partial h}{\partial z_{0}}\right)\right)\right)_{0}
\end{aligned}
$$

Pelo item $i i), \lambda_{h, z}^{0}(0)=\left(\Gamma_{h, z}^{1} \cdot V\left(\frac{\partial h}{\partial z_{0}}+j a z_{0}^{j-1}\right)\right)_{0}$

Daí, concluímos que $\left(\Gamma_{h+a w^{j}, w-z_{0}}^{1} \cdot V\left(h+a w^{j}\right)\right)_{0}=\lambda_{h, z}^{0}(0)+(j-1) \lambda_{h, z}^{0}(0)=j \lambda_{h, z}^{0}(0)$.

Observemos, por 4.4iii), que a modificação feita na função $h$ resulta numa aplicação que tem conjunto crítico de dimensão um a menos que a do conjunto crítico de $h$. Procedendo indutivamente, chega-se no caso de singularidade isolada.

Teorema 4.5. Sejam $h:(U, 0) \rightarrow(\mathbb{C}, 0)$ uma função analítica com $U \subseteq \mathbb{C}^{n+1}$ aberto e $s=$ $\operatorname{dim}_{0} \Sigma(h)$ com $s \geq 1$. Considere $z=\left(z_{0}, \ldots, z_{n}\right)$ uma escolha linear de coordenadas tais que $\lambda_{h, z}^{i}(0)$ está definido para todo $i \leq s$. Seja a um número complexo não nulo e use as coordenadas $\widetilde{z}=\left(z_{1}, \ldots, z_{n}, z_{0}\right)$ para $h+a z_{0}^{j}$, onde $j \geq 2$.

Se j é maior ou igual ao raio polar máximo de hentão, para todos exceto um número finito de complexos $a$, 
i) $\Sigma\left(h+a z_{0}^{j}\right)=\Sigma(h) \cap V\left(z_{0}\right)$ como germes de conjuntos em 0 ;

ii) $\operatorname{dim}_{0} \Sigma\left(h+a z_{0}^{j}\right)=s-1$;

iii) $\lambda_{h+a z_{0}^{j}, \tilde{z}}^{i}(0)$ existe para todo $i \leq s-1$;

iv) $\lambda_{h+a z_{0}^{j}, \tilde{z}}^{0}(0)=\lambda_{h, z}^{0}(0)+(j-1) \lambda_{h, z}^{1}(0)$

v) $\lambda_{h+a z_{0}^{j}, z^{i}}^{i}(0)=(j-1) \lambda_{h, z}^{i+1}(0)$, para $1 \leq i \leq s-1$.

Além disso, se jé estritamente maior que o raio polar máximo de h, as igualdades acima mantêm-se para todo a não nulo. Em particular, esse é o caso se $j \geq 2+\lambda_{h, z}^{0}(0)$.

\section{Demonstração.}

$$
\begin{aligned}
\lambda_{h+a z_{0}^{j}, \tilde{z}}^{0}(0) & =\left(\Gamma_{h+a z_{0}^{j}, \tilde{z}}^{1} \cdot V\left(\frac{\partial h}{\partial z_{1}}\right)\right)_{0}=\left(\Gamma_{h, z}^{2} \cdot V\left(\frac{\partial h}{\partial z_{0}}+j a z_{0}^{j-1}\right) \cdot V\left(\frac{\partial h}{\partial z_{1}}\right)\right)_{0} \\
& =\left(\left(\Gamma_{h, z}^{1}+\Lambda_{h, z}^{1}\right) \cdot V\left(\frac{\partial h}{\partial z_{0}}+j a z_{0}^{j-1}\right)\right)_{0} \\
& =\left(\Gamma_{h, z}^{1} \cdot V\left(\frac{\partial h}{\partial z_{0}}+j a z_{0}^{j-1}\right)\right)_{0}+(j-1)\left(\Lambda_{h, z}^{1} \cdot V\left(z_{0}\right)\right)_{0}
\end{aligned}
$$

e, aplicando o Lema 4.4ii),

$$
=\lambda_{h, z}^{0}(0)+(j-1) \lambda_{h, z}^{1}(0) .
$$

Pelo Lema 4.4iv),

$$
\Gamma_{h+a z_{0}^{j}, \tilde{z}}^{i}=\Gamma_{h, z}^{i+1} \cdot V\left(\frac{\partial h}{\partial z_{0}}+j a z_{0}^{j-1}\right), \forall i \geq 1
$$


Então,

$$
\begin{aligned}
\Gamma_{h+a z_{0}^{j}, \tilde{z}}^{i}+\Lambda_{h+a z_{0}^{j}, \tilde{z}}^{i} & =\Gamma_{h+a z_{0}^{j}, \tilde{z}}^{i+1} \cdot V\left(\frac{\partial h}{\partial z_{i+1}}\right)=\Gamma_{h, z}^{i+2} \cdot V\left(\frac{\partial h}{\partial z_{0}}+j a z_{0}^{j-1}\right) \cdot V\left(\frac{\partial h}{\partial z_{i+1}}\right) \\
& =\left(\Gamma_{h, z}^{i+1}+\Lambda_{h, z}^{i+1}\right) \cdot V\left(\frac{\partial h}{\partial z_{0}}+j a z_{0}^{j-1}\right) \\
& =\left(\Gamma_{h, z}^{i+1} \cdot V\left(\frac{\partial h}{\partial z_{0}}+j a z_{0}^{j-1}\right)\right)+(j-1)\left(\Lambda_{h, z}^{i+1} \cdot V\left(z_{0}\right)\right) \\
& =\Gamma_{h+a z_{0}^{j}, \tilde{z}}^{i}+(j-1)\left(\Lambda_{h, z}^{i+1} \cdot V\left(z_{0}\right)\right) .
\end{aligned}
$$

Cancelando $\Gamma_{h+a z_{0}^{j}, \tilde{z}}^{i}$ em cada um dos lados da equação temos que

$$
\Lambda_{h+a z_{0}^{j}, \widetilde{z}}^{i}=(j-1)\left(\Lambda_{h, z}^{i+1} \cdot V\left(z_{0}\right)\right)
$$

Portanto,

$$
\lambda_{h+a z_{0}^{j}, \tilde{z}}^{i}(0)=\left(\Lambda_{h+a z_{0}^{j}, \tilde{z}}^{i} \cdot V\left(z_{1}, \ldots, z_{i}\right)\right)_{0}=(j-1)\left(\Lambda_{h, z}^{i+1} \cdot V\left(z_{0}\right) \cdot V\left(z_{1}, \ldots, z_{i}\right)\right)_{0}=(j-1) \lambda_{h, z}^{i+1}
$$

Aplicando as fórmulas de Lê-Iomdine indutivamente concluímos

Corolário 4.6. Seja $h:(U, 0) \rightarrow(\mathbb{C}, 0)$ uma função analítica e $s=\operatorname{dim}_{0} \sum(h)$. Seja $z=$ $\left(z_{0}, \ldots, z_{n}\right)$ uma escolha linear de coordenadas tal que $\lambda_{h, z}^{i}(0)$ está definido para todo $i \leq s$. Então, para $0 \ll j_{0} \ll j_{1} \ll \cdots \ll j_{s-1}$,

$$
h+z_{0}^{j_{0}}+z_{1}^{j_{1}}+\cdots+z_{s-1}^{j_{s-1}}
$$

tem singularidade isolada na origem e seu número de Milnor é dado por

$$
\mu\left(h+z_{0}^{j_{0}}+z_{1}^{j_{1}}+\cdots+z_{s-1}^{j_{s-1}}\right)=\sum_{i=0}^{s}\left(\prod_{k=0}^{i-1}\left(j_{k}-1\right)\right) \lambda_{h, z}^{i}(0) .
$$




\subsection{Fórmula de Plücker e característica de Euler}

Uma outra aplicação das fórmulas de Lê-Iomdine é a fórmula de Plücker para polinômios homogêneos.

Proposição 4.7. (Fórmula de Plücker) Sejam h um polinômio homogêneo de grau d em $n+1$ variáveis e $s=\operatorname{dim}_{0} \Sigma(h)$. Suponha que $\lambda_{h, z}^{i}(0)$ existe para todo $i \leq s$. Então

$$
\sum_{i=0}^{s}(d-1)^{i} \lambda_{h, z}^{i}(0)=(d-1)^{n+1}
$$

Demonstração. Pela Observação 4.3, o raio polar máximo de $h$ é $d$. Consideremos $f:=$ $h+z_{0}^{d}+z_{1}^{d}+\cdots+z_{s-1}^{d}$. Pelo Corolário 4.6 temos

$$
\mu(f)=\sum_{i=0}^{s}\left(\prod_{k=0}^{i-1}(d-1)\right) \lambda_{h, z}^{i}(0)=\sum_{i=0}^{s}(d-1)^{i} \lambda_{h, z}^{i}(0)
$$

Agora, pelo Teorema 2.18, $\mu(f)=(d-1)^{n+1}$. Portanto, $\sum_{i=0}^{s}(d-1)^{i} \lambda_{h, z}^{i}(0)=(d-1)^{n+1}$.

A Proposição 4.7 é, em geral, usado para calcular a característica de Euler da fibra de Milnor de um polinômio homogêneo.

Definição 4.8. Seja $M$ uma variedade. A característica de Euler de $M, \chi(M)$, é definida por

$$
\chi(M)=\sum_{j}(-1)^{j} \text { posto } H_{j}(M)
$$

Em particular, quando $f:\left(U \subseteq \mathbb{C}^{n+1}, 0\right) \rightarrow \mathbb{C}$ é uma função analítica com ponto crítico isolado na origem e $M=F_{f, 0}$ temos que $\chi\left(F_{f, 0}\right)=1+(-1)^{n} \mu(f)$.

Do teorema 3.3 de [14] a característica de Euler reduzida da fibra de Milnor de $h$ em 0, denotada por $\tilde{\chi}\left(F_{h, 0}\right)$, é definida por

$$
\widetilde{\chi}\left(F_{h, 0}\right):=\sum_{i=0}^{s}(-1)^{n-i} \lambda_{h, z}^{i}(0)
$$


onde $s=\operatorname{dim}_{0} \Sigma(f)$.

No caso em que $h$ é um polinômio homogêneo de grau $d$ com conjunto crítico 1-dimensional, $\chi\left(F_{h, 0}\right):=1+(-1)^{n} \lambda_{h, z}^{0}(0)+(-1)^{n-1} \lambda_{h, z}^{1}(0)$ e pelo corolário 4.7 temos que $(d-1)^{n+1}=$ $\lambda_{h, z}^{0}(0)+(d-1) \lambda_{h, z}^{1}(0)$. Logo, se sabemos o grau e $\lambda_{h, z}^{1}(0)$ então sabemos $\lambda_{h, z}^{0}(0)$, e daí, a característica de Euler.

Ser capaz de calcular a característica de Euler da fibra de Milnor de um polinômio homogêneo implica, em muitos casos, calcular a característica de Euler da fibra de Milnor de um polinômio quase homogêneo.

Para ver isso, seja $f:\left(\mathbb{C}^{n+1}, 0\right) \rightarrow(\mathbb{C}, 0)$ um polinômio quase homogêneo. Então existem inteiros $r_{0}, \ldots, r_{n}$ tais que se $\pi: \mathbb{C}^{n+1} \rightarrow \mathbb{C}^{n+1}$ é dada por $\pi\left(z_{0}, \ldots, z_{n}\right)=\left(z_{0}^{r_{0}}, \ldots, z_{n}^{r_{n}}\right)$ então $h:=$ $f \circ \pi$ é polinômio homogêneo.

Além disso, a restrição de $\pi$ induz uma aplicação da fibra de Milnor $F_{h, 0}$ de $h$ na origem à fibra de Milnor $F_{f, 0}$ de $f$ na origem.

Denote tal aplicação por $\tilde{\pi}: F_{h, 0} \rightarrow F_{f, 0}$.

Agora considere a estratificação de $\mathbb{C}^{n+1}$ derivada das combinações dos hiperplanos dada por todos hiperplanos coordenados, isto é, seja $I$ o conjunto de índices $\{0, \ldots, n\}$ e para $J \subseteq I$ seja $w_{J}$ a intersecção dos hiperplanos dada por $w_{J}:=V\left(z_{j} \mid j \in J\right)$ e seja $S_{J}$ o estrato de Whitney $S_{J}:=w_{J}-\bigcup_{J \varsubsetneqq K} w_{K}$

A estratificação $\left\{S_{J}\right\}$ determina estratificações de Whitney $\left\{S_{J} \cap F_{h, 0}\right\}$ e $\left\{S_{J} \cap F_{f, 0}\right\}$ de $F_{h, 0}$ e $F_{f, 0}$, respectivamente e com essas estratificações $\tilde{\pi}$ torna-se uma aplicação estratificada.

Definição 4.9. Seja $p: E \rightarrow B$ uma aplicação contínua e sobrejetora. Dizemos que p é uma função de recobrimento se cada ponto $b \in B$ possui uma vizinhança $U_{b}$ eventualmente coberta por $p$, ou seja, existem conjuntos abertos $V_{\alpha}$ disjuntos, tais que $p: V_{\alpha} \rightarrow U_{b}$ é homeomorfismo.

Além disso, a restrição de $\widetilde{\pi}$ a aplicação de $\left\{S_{J} \cap F_{h, 0}\right\}$ a $\left\{S_{J} \cap F_{f, 0}\right\}$ é uma aplicação de recobrimento topológico com fibra igual a $\prod_{i \notin J} r_{i}$ pontos. (Para maiores detalhes sobre aplicações estratificadas e aplicações de recobrimento ver Gaffney [5]). 
Daí, $\chi\left(F_{h, 0}\right)=\sum_{J} \chi\left(S_{J} \cap F_{h, 0}\right)=\sum_{J}\left(\prod_{i \notin J} r_{i}\right) \chi\left(S_{J} \cap F_{f, 0}\right)$

e, com algumas operações,

$\sum_{J} c_{J}\left(\sum_{J \subseteq K} \chi\left(S_{K} \cap F_{f, 0}\right)\right)$, onde $c_{J}=(-1)^{|J|} \sum_{L \subseteq J}\left((-1)^{|L|} \prod_{i \notin L} r_{i}\right)$.

A vantagem desta última fórmula é que $\sum_{J \subseteq K} \chi\left(S_{K} \cap F_{f, 0}\right)=\chi\left(F_{f_{w_{J}}}, 0\right)$.

Por isso, $\chi\left(F_{h, 0}\right)=\sum_{J} c_{J} \chi\left(F_{f_{\left.\right|_{J}}, 0}\right)$.

$$
\begin{aligned}
\chi\left(F_{h, 0}\right) & =\chi\left(F_{f, 0}\right)\left(r_{0} \cdots r_{n}\right)+\sum_{J \neq \varnothing} c_{J} \chi\left(F_{f_{\left.\right|_{J}}, 0}\right) \\
\Rightarrow \chi\left(F_{f, 0}\right) & =\frac{\chi\left(F_{h, 0}\right)-\sum_{J \neq \varnothing} c_{J} \chi\left(F_{f_{\left.\right|_{J}}, 0}\right)}{r_{0} \cdots r_{n}} .
\end{aligned}
$$

Notemos que

1. Essa fórmula é indutivamente fácil, se $J \neq \varnothing$, quando $f_{\left.\right|_{w_{J}}}$ é um polinômio quase homogêneo em menos variáveis.

2. Note que, nesta fórmula, não precisamos considerar o termo quando $J=\{0, \ldots, n\}$ pois $w_{J}=\{$ origem $\}$ e daí $\chi\left(F_{f_{w_{J}}}, 0\right)=0$.

3. Isso é particularmente útil no caso onde $f$ é um polinômio quase homogêneo com conjunto crítico 1-dimensional e cada restrição a uma superfície plana, $f_{\left.\right|_{w_{J}}}$, também tem um conjunto crítico 1-dimensional (ou 0-dimensional).

Exemplo 4.10. Seja $h$ uma aplicação analítica nas variáveis $x$ e $y$ e suponha que $h=P \prod Q_{i}^{\alpha_{i}}$, onde $P$ e $\prod Q_{i}^{\alpha_{i}}$ são relativamente primos e $\alpha_{i} \geq 2$, isto é, $h=0$ é uma curva singular não reduzida. Desejamos calcular os números de Lê de $h$ na origem.

Sejam $z_{0}=a x+b y$, onde $a \neq 0$ e $z_{1}=y$. Então $x=\frac{z_{0}}{a}-\frac{b}{a} z_{1}$ e $y=z_{1}$.

$$
\begin{aligned}
\Lambda_{h, z}^{1}+\Gamma_{h, z}^{1} & =\left[V\left(\frac{\partial h}{\partial z_{1}}\right)\right]=\left[V\left(\frac{\partial h}{\partial x}\left(\frac{-b}{a}\right)+\frac{\partial h}{\partial y}\right)\right] \\
& =\sum\left[V\left(Q_{i}^{\alpha_{i}-1}\right)\right]+\left[V\left(\frac{\frac{\partial h}{\partial x}\left(\frac{-b}{a}\right)+\frac{\partial h}{\partial y}}{\prod Q_{i}^{\alpha_{i}-1}}\right)\right] .
\end{aligned}
$$


Assim, como $\left[V\left(\frac{\frac{\partial h}{\partial x}\left(\frac{-b}{a}\right)+\frac{\partial h}{\partial y}}{\prod Q_{i}^{\alpha_{i}-1}}\right)\right]$ não tem componente contida no conjunto crítico de $h$ (esse é o caso para uma escolha genérica de $(a, b)$ ), temos que

$$
\begin{gathered}
\lambda_{h, z}^{1,(0)}=\sum\left(\alpha_{i}-1\right)\left(V\left(Q_{i}\right) \cdot V(a x+b y)\right)_{0} \\
\lambda_{h, z}^{0}(0)=\left(V\left(\frac{\frac{\partial h}{\partial x}\left(\frac{-b}{a}\right)+\frac{\partial h}{\partial y}}{\prod Q_{i}^{\alpha_{i}-1}}\right) \cdot V\left(\frac{\partial h}{\partial x}\right)\right)_{0},
\end{gathered}
$$

onde usamos que $V\left(\frac{\partial h}{\partial z_{0}}\right)=V\left(\frac{\partial h}{\partial x}\right)$.

Exemplo 4.11. Consideremos o caso de uma singularidade de uma curva plana quase homogênea. Suponha que a fatoração irredutível de $f\left(z_{0}, z_{1}\right)=z_{0}^{a} z_{1}^{b} \Pi f_{i}^{m_{i}}$ onde pode ocorrer o caso $a$ ou $b$ igual a zero. Sejam $\pi\left(z_{0}, z_{1}\right)=\left(z_{0}^{r_{0}}, z_{1}^{r_{1}}\right), h$ o polinômio homogêneo $f \circ \pi$ e $h_{i}$ o polinômio homogêneo $f_{i} \circ \pi$. Seja $d$ o grau de $h$ e $d_{i}$ o grau de $h_{i}$. Assim,

$$
\begin{gathered}
\chi\left(F_{f, 0}\right)=\frac{\chi\left(F_{h, 0}\right)-c_{0} \chi\left(F_{f_{w_{0}}, 0}\right)-c_{1} \chi\left(F_{f_{w_{1}}, 0}\right)}{r_{0} r_{1}} \\
\Rightarrow \chi\left(F_{f, 0}\right)=\frac{\chi\left(F_{h, 0}\right)+r_{1}\left(r_{0}-1\right) \chi\left(F_{f_{V\left(z_{0}\right)}, 0}\right)+r_{0}\left(r_{1}-1\right) \chi\left(F_{f_{\mid V\left(z_{1}\right)}, 0}\right)}{r_{0} r_{1}} . \\
\text { Agora, } \chi\left(F_{f_{\mid V\left(z_{k}\right)}, 0}\right)=\left\{\begin{array}{ccc}
0 & \text { se } & f_{\left.\right|_{V\left(z_{k}\right)}} \equiv 0 ; \\
\text { multiplicidade de } f_{\left.\right|_{V\left(z_{k}\right)}} & , & \text { caso contrário. }
\end{array}\right.
\end{gathered}
$$

Além disso, como $h$ é homogênea, podemos calcular $\chi\left(F_{h, 0}\right)$ do Corolário 4.6 sabendo somente $\lambda_{h}^{1}(0)$, que pode ser calculado como no Exemplo 4.10 . 
Assim, $\quad r_{0} r_{1} \chi\left(F_{f, 0}\right)=\left\{\begin{array}{rcc}-d \sum d_{i} & \text { se } & a \neq 0, b \neq 0 ; \\ d\left(r_{0}-\sum d_{i}\right) & \text { se } & a=0, b \neq 0 ; \\ d\left(r_{1}-\sum d_{i}\right) & \text { se } & a \neq 0, b=0 ; \\ d\left(r_{0}+r_{1}-\sum d_{i}\right) & \text { se } & a=b=0 .\end{array}\right.$

Exemplo 4.12. Consideremos a singularidade "rabo de andorinha". Essa é dada como o conjunto de zeros de $f=256 z_{0}^{3}-27 z_{1}^{4}-128 z_{0}^{2} z_{2}^{2}+144 z_{0} z_{1}^{2} z_{2}+16 z_{0} z_{2}^{4}-4 z_{1}^{2} z_{2}^{3}$.

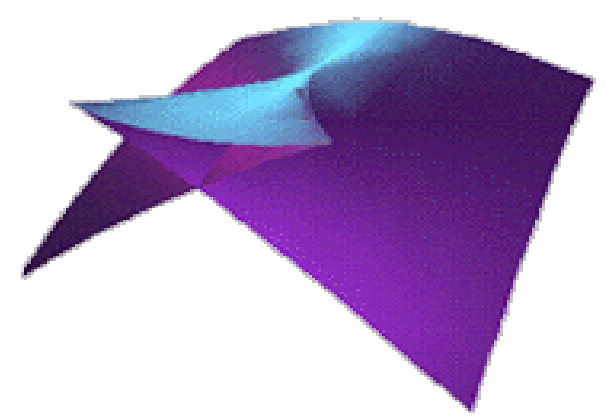

Figura 4.1: singularidade "rabo de andorinha"

Se $\pi\left(z_{0}, z_{1}, z_{2}\right)=\left(z_{0}^{4}, z_{1}^{3}, z_{2}^{2}\right)$ então $h=f \circ \pi=256 z_{0}^{12}-27 z_{1}^{12}-128 z_{0}^{8} z_{2}^{4}+144 z_{0}^{4} z_{1}^{6} z_{2}^{2}+16 z_{0}^{4} z_{2}^{8}-$ $4 z_{1}^{6} z_{2}^{6}$ é um polinômio homogêneo de grau 12 .

Neste caso temos que $I=\{0,1,2\}$, os possíveis $J$ são $\{0\},\{1\},\{2\},\{0,1\},\{0,2\}$ e $\{1,2\}$ e $r_{0}=4, r_{1}=3$ e $r_{2}=2$.

$$
\begin{aligned}
& \left\{\begin{array}{l}
f_{\left.\right|_{w_{\{0\}}}}=f_{\left.\right|_{V\left(z_{0}\right)}}=-27 z_{1}^{4}-4 z_{1}^{2} z_{2}^{3}=z_{1}^{2}\left(-27 z_{1}^{2}-4 z_{2}^{3}\right) \Rightarrow \chi\left(F_{f_{w_{\{0\}}}, 0}\right)=-8 \\
c_{\{0\}}=(-1)^{1}\left((-1)^{0} r_{0} r_{1} r_{2}+(-1)^{1} r_{1} r_{2}\right)=-18
\end{array}\right. \\
& \left\{\begin{array}{l}
f_{\left.\right|_{w_{\{1\}}}}=f_{\left.\right|_{V\left(z_{1}\right)}}=256 z_{0}^{3}-128 z_{0}^{2} z_{2}^{2}+16 z_{0} z_{2}^{4}=16 z_{0}\left(4 z_{0}-z_{2}^{2}\right)^{2} \Rightarrow \chi\left(F_{f_{\left.\right|_{\{1\}}}, 0}\right)=-3 \\
c_{\{1\}}=(-1)^{1}\left((-1)^{0} r_{0} r_{1} r_{2}+(-1)^{1} r_{0} r_{2}\right)=-16
\end{array}\right.
\end{aligned}
$$




$$
\begin{aligned}
& \left\{\begin{array}{l}
f_{\left.\right|_{w_{\{2\}}}}=f_{\left.\right|_{V\left(z_{2}\right)}}=256 z_{0}^{3}-27 z_{1}^{4} \Rightarrow \chi\left(F_{f_{\left.\right|_{\{2\}}}, 0}\right)=-5 \\
c_{\{2\}}=(-1)^{1}\left((-1)^{0} r_{0} r_{1} r_{2}+(-1)^{1} r_{0} r_{1}\right)=-12
\end{array}\right. \\
& f_{\left.\right|_{w_{\{0,1\}}}}=f_{\left.\right|_{V\left(z_{0}, z_{1}\right)}} \equiv 0 \Rightarrow \chi\left(F_{f_{\left.\right|_{\{1\}}}, 0}\right)=0 \\
& \left\{\begin{array}{l}
f_{\left.\right|_{w_{\{0,2\}}}}=f_{\left.\right|_{V\left(z_{0}, z_{2}\right)}}=-27 z_{1}^{4} \Rightarrow \chi\left(F_{f_{\left.\right|_{\{}\{0,2\}}, 0}\right)=4 \\
c_{\{0,2\}}=(-1)^{2}\left((-1)^{0} r_{0} r_{1} r_{2}+(-1)^{1} r_{1} r_{2}+(-1)^{1} r_{0} r_{1}+(-1)^{2} r_{1}\right)=9
\end{array}\right. \\
& \left\{\begin{array}{l}
f_{\left.\right|_{w_{\{1,2\}}}}=f_{\left.\right|_{V\left(z_{1}, z_{2}\right)}}=256 z_{0}^{3} \Rightarrow \chi\left(F_{f_{\left.\left.\right|_{\{1}, 2\right\}}, 0}\right)=3 \\
c_{\{1,2\}}=(-1)^{2}\left((-1)^{0} r_{0} r_{1} r_{2}+(-1)^{1} r_{0} r_{2}+(-1)^{1} r_{0} r_{1}+(-1)^{2} r_{0}\right)=8
\end{array}\right. \\
& \left(r_{0} r_{1} r_{2}\right) \chi\left(F_{f, 0}\right)=\chi\left(F_{h, 0}\right)-c_{\{0\}} \chi\left(F_{f_{\left.\right|_{\{0\}}}, 0}\right)-c_{\{1\}} \chi\left(F_{f_{\left.\right|_{\{1\}}}, 0}\right)-c_{\{2\}} \chi\left(F_{f_{\left.\right|_{\{}}, 0}, 0\right. \\
& -c_{\{0,1\}} \chi\left(F_{f_{\left.\right|_{\{0,1\}}}, 0}\right)-c_{\{0,2\}} \chi\left(F_{f_{\left.\right|_{\{0,2\}}}, 0}\right)-c_{\{1,2\}} \chi\left(F_{f_{\left.\right|_{\{1,2\}}}, 0}\right) \\
& \Rightarrow \quad 24 \chi\left(F_{f, 0}\right)=\chi\left(F_{h, 0}\right)+18(-8)+16(-3)+12(-5)+0-9(4)-8(3) \\
& \Rightarrow \quad 24 \chi\left(F_{f, 0}\right) \quad=\chi\left(F_{h, 0}\right)-312 \text {. }
\end{aligned}
$$

Computacionalmente é obtido que $\operatorname{dim}_{0} \Sigma(h)=1$ e $\lambda_{h, z}^{1}(0)=83$.

Então, pelo Corolário 4.7, $\lambda_{h, z}^{0}(0)+11 \lambda_{h, z}^{1}(0)=(11)^{3} \Rightarrow \lambda_{h, z}^{0}(0)=418$.

$\chi\left(F_{h, 0}\right)=\lambda_{h, z}^{0}(0)-\lambda_{h, z}^{1}(0)+1=418-83+1=336$.

Portanto, $\chi\left(F_{f, 0}\right)=\frac{336-312}{24}=1$.

\subsection{Uso do Singular no cálculo dos números de Lê de um polinômio}

Nesta secção descrevemos como usar o programa computacional Singular [6] para calcular as multiplicidades de ideais em um anel de polinômios a partir de um conjunto de geradores, e daí calcular os números de Lê de um polinômio. Como aplicação mostramos como calcular 
a característica de Euler da fibra de Milnor no caso de um conjunto crítico 1 dimensional, se a função $h$, é um polinômio quase homogêneo ou não.

Seja $h$ um polinômio com conjunto singular 1 dimensional. Procedemos como segue para calcular os números de Lê, $\lambda^{0}$ e $\lambda^{1}$, na origem com respeito a um conjunto genérico de coordenadas.

Como já observamos, $\lambda^{1}$ é nada mais que a multiplicidade do jacobiano de $h$ na origem. Assim, o programa pode calculá-lo. Isso se dá da seguinte maneira:

$>$ LIB "sing.lib";

$>\operatorname{ring} r=0,\left(z_{1}, \ldots, z_{n}\right), \mathrm{ds} ; \quad$ (onde $\left(z_{1}, \ldots, z_{n}\right)$ são as variáveis de $h$ )

$>$ poly $h=$ definição de $h$;

$>$ ideal $\mathrm{i}=\mathrm{jacob}(\mathrm{h})$;

$>\mathrm{i}$; (para visualizar o jacobiano de $h$ )

$>$ ideal $\mathrm{j}=\operatorname{std}(\mathrm{i}) ; \quad$ (para obter a base standard(i) $)$

$>\mathrm{j}$

$>$ mult $(\mathrm{j})$;

Agora, consideramos um hiperplano que é genérico suficiente para que a multiplicidade do conjunto singular seja o número de intersecções (na origem) deste hiperplano com o conjunto singular.

Portanto, para obter $\lambda^{1}$, consideramos o hiperplano, $V(L)$, para alguma forma linear, $L$. Pelo Teorema 2.18 temos que para todo $k$ suficientemente grande, $h+L^{k}$ tem singularidade isolada na origem e o número de Milnor $\mu\left(h+L^{k}\right)$ é igual a $\lambda^{0}+(k-1) \lambda^{1}$.

Para calcular o número de Milnor procedemos da seguinte maneira:

$>$ LIB "sing.lib";

$>\operatorname{ring} r=0,\left(z_{1}, \ldots, z_{n}\right)$, ds; $\quad$ (onde $\left(z_{1}, \ldots, z_{n}\right)$ são as variáveis de $h+L^{k}$ )

$>$ poly $h+L^{k}=$ definição de $h+L^{k}$;

$>$ milnor $\left(h+L^{k}\right)$;

Temos um método efetivo para encontrar $k$ suficientemente grande tal que a fórmula de LêIomdine ocorra e, assim, podemos achar $\lambda^{0}$. 
Para encontrar este valor de $k$ apropriado, o primeiro passo é obter $h+L^{k}$ com singularidade isolada, e isto pode ser checado pelo cálculo computacional do número de Milnor acima.

A demonstração da fórmula de Lê-Iomdine no Teorema 4.5 mostra que a fórmula ocorre se

$$
\mu_{k}-(k-1) \lambda^{1} \leq k-2
$$

Por isso, para achar $\lambda^{0}$, iniciamos com um $k$ relativamente pequeno e checamos se ocorre

$$
\mu_{k} \leq k-2+(k-1) \lambda^{1}
$$

Se a desigualdade é falsa, troquemos por um $k$ maior até que a desigualdade ocorra e então

$$
\lambda^{0}=\mu_{k}-(k-1) \lambda^{1}
$$

Temos também um método alternativo para calcular não somente os números de Lê mas também o raio polar máximo de $h$.

Novamente, da demonstração do Teorema 4.5, se $\mu_{i}$ é como acima, então $\mu_{k+1}-\mu_{k}=\lambda^{1}$ se, e somente se, $k \geq$ raio polar máximo, obtendo desta forma um método simples para o cálculo do raio polar.

Daí, para achar o raio polar máximo, calcula-se $\mu_{k}$ para sucessivos valores de $k$ - procurando um tal que a diferença dê $\lambda^{1}$.

Uma vez que isso ocorre, $k \geq$ raio polar máximo e, como antes, concluímos que $\lambda^{0}=$ $\mu_{k}-(k-1) \lambda^{1}$

Embora esse método recorra ao cálculo de no mínimo 2 números de Milnor, $\mu_{k}$, ele ainda é um caminho mais eficiente para calcular $\lambda^{0}$ - fornecendo assim uma forma de calcular o valor $j \geq 2+\lambda^{0}$ obtido no Teorema 4.5.

Exemplo 4.13. Considere novamente o "rabo de andorinha" do Exemplo 4.12 definido por $h=256 x^{3}-27 y^{4}-128 x^{2} z^{2}+144 x y^{2} z+16 x z^{4}-4 y^{2} z^{3}$.

Pelo programa computacional Singular temos

$>$ LIB "sing.lib";

$>\operatorname{ring} r=0,(x, y, z), \mathrm{ds}$; 
$>$ poly $h=256 x^{3}-27 y^{4}-128 x^{2} z^{2}+144 x y^{2} z+16 x z^{4}-4 y^{2} z^{3}$;

$>$ ideal $\mathrm{i}=\mathrm{jacob}(\mathrm{h})$;

$>\mathrm{i}$

$i[1]=768 x^{2}+144 y^{2} z-256 x z^{2}+16 z^{4}$

$i[2]=-108 y^{3}+288 x y z-8 y z^{3}$

$i[3]=144 x y^{2}-256 x^{2} z-12 y^{2} z^{2}+64 x z^{3}$

$>$ ideal $\mathrm{j}=\operatorname{std}(\mathrm{i})$;

$>\mathrm{j}$

$j[1]=48 x^{2}+9 y^{2} z-16 x z^{2}+z^{4}$

$j[2]=36 x y^{2}-64 x^{2} z-3 y^{2} z^{2}+16 x z^{3}$

$j[3]=27 y^{3}-72 x y z+2 y z^{3}$

$>$ mult $(\mathrm{j})$;

5

Alternativamente, sabemos que as singularidades do "rabo de andorinha" consistem de uma curva suave de pontos duplos com multiplicidade 1 mais uma curva de cúspide de multiplicidade 2, daí, a multiplicidade do jacobiano na origem $=1+2.2=5$.

Sendo $L=z$, pelo programa computacional Singular temos

$>$ LIB "sing.lib";

$>\operatorname{ring} r=0,(x, y, z), \mathrm{ds}$;

$>$ poly $h+L^{2}=256 x^{3}-27 y^{4}-128 x^{2} z^{2}+144 x y^{2} z+16 x z^{4}-4 y^{2} z^{3}+z^{2}$;

$>$ milnor $\left(h+L^{2}\right)$;

6

$>$ poly $h+L^{3}=256 x^{3}-27 y^{4}-128 x^{2} z^{2}+144 x y^{2} z+16 x z^{4}-4 y^{2} z^{3}+z^{3}$;

$>$ milnor $\left(h+L^{3}\right)$;

12

$>$ poly $h+L^{4}=256 x^{3}-27 y^{4}-128 x^{2} z^{2}+144 x y^{2} z+16 x z^{4}-4 y^{2} z^{3}+z^{4}$;

$>$ milnor $\left(h+L^{4}\right)$;

18

$>$ poly $h+L^{5}=256 x^{3}-27 y^{4}-128 x^{2} z^{2}+144 x y^{2} z+16 x z^{4}-4 y^{2} z^{3}+z^{5}$;

$>$ milnor $\left(h+L^{5}\right)$; 
24

$>$ poly $h+L^{6}=256 x^{3}-27 y^{4}-128 x^{2} z^{2}+144 x y^{2} z+16 x z^{4}-4 y^{2} z^{3}+z^{6}$;

$>$ milnor $\left(h+L^{6}\right)$;

30

$>$ poly $h+L^{7}=256 x^{3}-27 y^{4}-128 x^{2} z^{2}+144 x y^{2} z+16 x z^{4}-4 y^{2} z^{3}+z^{7}$;

$>$ milnor $\left(h+L^{7}\right)$;

35

Agora, usando a notação acima, achamos

\begin{tabular}{|l|l|l|l|l|l|l|}
\hline$k$ & 2 & 3 & 4 & 5 & 6 & 7 \\
\hline$\mu_{k}$ & 6 & 12 & 18 & 24 & 30 & 35 \\
\hline
\end{tabular}

Da tabela, vemos que o raio polar máximo é no máximo $6, \lambda^{1}$ é, de fato, igual a 5 e $\lambda^{0}=$ $30-(6-1) 5=5$. Daí, a característica da fibra de Milnor de $h$ é igual a $\lambda^{0}-\lambda^{1}+1=1$, que está de acordo com nosso cálculo anterior.

\subsection{Fórmulas de Lê-Iomdine generalizadas}

Nesta secção, mostramos uma versão uniforme das fórmulas de Lê-Iomdine para famílias a 1-parâmetro de germes de hipersuperfícies singulares na origem.

Consideramos $\stackrel{\circ}{\mathbb{D}}$ um disco aberto ao redor da origem em $\mathbb{C}, U$ uma vizinhança aberta da origem em $\mathbb{C}^{n+1}$ e $f:(\stackrel{\circ}{\mathbb{D}} \times U, \stackrel{\circ}{\mathbb{D}} \times 0) \rightarrow(\mathbb{C}, 0)$ uma função analítica. Naturalmente, escrevemos $f_{t}$ para a função definida por $f_{t}(z):=f(t, z)$.

Primeiro, necessitamos do Lema 4.15. Para demonstrar este lema, precisamos da seguinte proposição, demonstrada em [15].

Proposição 4.14. Dada uma função analítica $f:(X, 0) \rightarrow(\mathbb{C}, 0)$ e uma estratificação $\mathscr{B}=\left\{S_{\alpha}\right\}$ de $X$, existe uma vizinhança da origem na qual $\Sigma_{\mathscr{B}}(f) \subseteq f^{-1}(0)$, onde $\Sigma_{\mathscr{B}}(f)=\bigcup_{\alpha} \Sigma\left(f_{\left.\right|_{S_{\alpha}}}\right)$.

Lema 4.15. Para todo i e para todo $p=\left(t_{0}, z_{0}, \ldots, z_{n}\right)$ próximo da origem tal que $t_{0} \neq 0$,

$$
\Gamma_{f_{t_{0}}, z}^{i}=\Gamma_{f,(t, z)}^{i+1} \cap V\left(t-t_{0}\right)=\Gamma_{f,(t, z)}^{i+1} \cdot V\left(t-t_{0}\right)
$$


como ciclos em p, sem se importar quão genéricas as coordenadas $\left(t, z_{0}, \ldots, z_{n}\right)$ podem ser.

Demonstração. Fixe uma boa estratificação, $\mathscr{B}=\left\{S_{\alpha}\right\}$, para $f$ na origem. Considere a aplicação $g\left(t, z_{0}, \ldots, z_{n}\right)=t$. Pela Proposição $4.14 \bigcup_{\alpha} \Sigma\left(g_{\left.\right|_{\alpha}}\right) \subseteq g^{-1}(0)$. Daí numa vizinhança da origem, a aplicação $g$ restrita a cada estrato de $\mathscr{B}$ pode ter somente 0 como valor crítico.

Portanto, para todo $t_{0} \neq 0, V\left(t-t_{0}\right)$ é um pedaço pré-polar de $f$ em $p$. Em particular, $\Sigma(f) \cap V\left(t-t_{0}\right)=\Sigma\left(f_{\left.\right|_{V\left(t-t_{0}\right)}}\right)=\Sigma\left(f_{t_{0}}\right)$.

Assim,

$$
\begin{aligned}
\Gamma_{f_{t_{0}, z}}^{i} & =V\left(t-t_{0}, \frac{\partial f}{\partial z_{i}}, \ldots, \frac{\partial f}{\partial z_{n}}\right) / \Sigma\left(f_{t_{0}}\right)=V\left(t-t_{0}, \frac{\partial f}{\partial z_{i}}, \ldots, \frac{\partial f}{\partial z_{n}}\right) / \Sigma(f) \cap V\left(t-t_{0}\right) \\
& =V\left(t-t_{0}, \frac{\partial f}{\partial z_{i}}, \ldots, \frac{\partial f}{\partial z_{n}}\right) / \Sigma(f)=\left(V\left(t-t_{0}\right) \cap V\left(\frac{\partial f}{\partial z_{i}}, \ldots, \frac{\partial f}{\partial z_{n}}\right)\right) / \Sigma(f) \\
& =\left(V\left(t-t_{0}\right) \cap V\left(\frac{\partial f}{\partial z_{i}}, \ldots, \frac{\partial f}{\partial z_{n}}\right) / \Sigma(f)\right) / \Sigma(f)=\left(\Gamma_{f,(t, z)}^{i+1} \cap V\left(t-t_{0}\right)\right) / \Sigma(f) .
\end{aligned}
$$

Mas, afirmamos que isso é igual a $\Gamma_{f,(t, z)}^{i+1} \cap V\left(t-t_{0}\right)$ a menos de subvariedade mergulhada para todo $t_{0} \neq 0$ pequeno.

Por outro lado, $\Gamma_{f,(t, z)}^{i+1} \cap V\left(t-t_{0}\right)$ teria uma componente contida em $\Sigma(f)$ para um número infinito de pequenos $t_{0}$, que implicaria que $\Gamma_{f,(t, z)}^{i+1}$ tem uma componente contida em $\Sigma(f)$ - uma contradição da definição de $\Gamma_{t,(t, z)}^{i+1}$.

Daí, $\Gamma_{f_{t_{0}}, z}^{i}=\Gamma_{f,(t, z)}^{i+1} \cap V\left(t-t_{0}\right)$ como subvariedade mergulhada e a conclusão segue.

Como no Teorema 4.5, quando usamos as coordenadas $z=\left(z_{0}, \ldots, z_{n}\right)$ para $f_{t}$, usamos as coordenadas $\widetilde{z}=\left(z_{1}, \ldots, z_{n}, z_{0}\right)$ para $f_{t}+z_{0}{ }^{j}$.

Teorema 4.16. (Fórmulas de Lê-Iomdine uniformes) Seja $s:=\operatorname{dim}_{0} \Sigma\left(f_{0}\right)$ e suponha $s \geq 1$. Suponha que $\lambda_{f_{t}, z}^{i}(0)$ está definido para todo $i \leq s$ e para todo t pequeno. Então, existe $r>0 e$ $j_{0}$ tais que, para todo $j \geq j_{0}$ e para todo $t \in \stackrel{\circ}{\mathbb{D}}_{r}$,

i) $\Sigma\left(f_{t}+z_{0}^{j}\right)=\Sigma\left(f_{t}\right) \cap V\left(z_{0}\right)$ como germes de conjuntos em 0 ; 
ii) $\operatorname{dim}_{0} \Sigma\left(f_{0}+z_{0}^{j}\right)=s-1$,

iii) $\lambda_{f_{t}+z_{0}^{j}, \tilde{z}}(0)$ está definido para todo $i \leq s-1$

iv) $\lambda_{f_{t}+z_{0}^{j}, z^{z}}^{0}(0)=\lambda_{f_{t}, z}^{0}(0)+(j-1) \lambda_{f_{t}, z}^{1}(0)$;

v) $\lambda_{f_{t}+z_{0}^{j}, z^{i}}(0)=(j-1) \lambda_{f_{t}, z}^{i+1}(0)$, para $1 \leq i \leq s-1$.

Demonstração. Pelo Teorema 4.5, se, em particular, $j \geq 2+\lambda_{f_{t}, z}^{0}(0)$ então as igualdades acima são satisfeitas. Então devemos mostrar que $\left\{\lambda_{f_{0}, z}^{0}(0)\right\}_{t_{0}}$ é limitada para $t_{0}$ pequeno. $\mathrm{O}$ que na verdade mostramos é que, para $t_{0} \neq 0, \lambda_{f_{t_{0}, z}}^{0}(0)$ independe de $t_{0}$.

Para $t_{0} \neq 0$ pequeno, podemos aplicar o Lema 4.15 para concluir

$$
\begin{aligned}
\Lambda_{f_{0}, z}^{0} & =\Gamma_{f_{0}, z}^{1} \cdot V\left(\frac{\partial f_{t_{0}}}{\partial z_{0}}\right)=\Gamma_{f_{t_{0}}, z}^{1} \cdot V\left(\frac{\partial f}{\partial z_{0}}\right) \\
& =\Gamma_{f,(t, z)}^{2} \cdot V\left(t-t_{0}\right) \cdot V\left(\frac{\partial f}{\partial z_{0}}\right)=V\left(t-t_{0}\right) \cdot\left(\Gamma_{f,(t, z)}^{2} \cdot V\left(\frac{\partial f}{\partial z_{0}}\right)\right) \\
& =V\left(t-t_{0}\right) \cdot\left(\Gamma_{f,(t, z)}^{1}+\Lambda_{f,(t, z)}^{1}\right)=V\left(t-t_{0}\right) \cdot \Lambda_{f,(t, z)}^{1} .
\end{aligned}
$$

Como, $\Lambda_{f,(t, z)}^{0}=\sum n_{v}[v]=\sum_{v \neq 0} n_{v}[v]+n_{0}[0]$ e $\Lambda_{f,(t, z)}^{0}$ tem dimensão 0, então $\Gamma_{f,(t, z)}^{1}$ coincide com $n_{0}[0]$, numa vizinhança de $\left(t_{0}, 0\right), \lambda_{f_{t_{0}, z}}^{0}(0)=\left(\eta_{r}[r] \cdot V\left(t-t_{0}\right)\right)_{\left(t_{0}, 0\right)}=\eta_{r}$ para todo $t_{0} \neq 0$ pequeno e com isso a conclusão segue.

Definição 4.17. Seja $\left\{f_{t}\right\}$ uma família de aplicações e $\left\{m\left(f_{t}\right)\right\}$ uma família de números inteiros e positivos associados. A família $\left\{m\left(f_{t}\right)\right\}$ satisfaz a propriedade da semicontinuidade superior (ou $\left\{m\left(f_{t}\right)\right\}$ é semicontínua superiormente) se $m\left(f_{t}\right) \leq m\left(f_{0}\right), \forall t \neq 0$.

No exemplo a seguir, mostramos que - diferente dos números de Milnor - os números de Lê em uma família não precisam ser semicontínuos superiormente.

Exemplo 4.18. Seja $f_{t}(x, y, z)=z^{2}-y^{3}-t x y^{2}$. As coordenadas $(x, y, z)$ são pré-polares na origem para $f_{t}$ para todo $t$. Fixamos esse conjunto de coordenadas.

Para $t_{0} \neq 0$, voltamos na situação do Exemplo 3.21, com $a=3$ e $b=2$; portanto $\lambda_{f_{t_{0}}}^{0}(0)=2$ e $\lambda_{f_{t_{0}}}^{1}(0)=1$. 
Por outro lado, a hipersuperfície definida por $f_{0}$ é uma singularidade produto de cruzamentos; daí, $\lambda_{f_{0}}^{0}(0)=0$ e $\lambda_{f_{0}}^{1}(0)=2$.

O próximo corolário mostra que essa situação não é completamente sem controle pois se temos uma família $f_{t}$, a $(s+1)$-upla de números de Lê $\left(\lambda_{f_{t}, z}^{s}(0), \lambda_{f_{t}, z}^{s-1}(0), \cdots, \lambda_{f_{t}, z}^{0}(0)\right)$ é lexicográfica semicontínua superiormente na variável $t$.

Corolário 4.19. Usando a notação do Teorema 4.16, a $(s+1)$-upla de números de Lê

$$
\left(\lambda_{f_{t}, z}^{s}(0), \lambda_{f_{t}, z}^{s-1}(0), \ldots, \lambda_{f_{t}, z}^{0}(0)\right)
$$

é lexicográfica semicontínua superiormente na variável $t$, isto é, para todo t pequeno, ou

$$
\begin{gathered}
\lambda_{f_{t}, z}^{s}(0)<\lambda_{f_{0}, z}^{s}(0) \\
\text { ou } \lambda_{f_{t}, z}^{s}(0)=\lambda_{f_{0}, z}^{s}(0) \text { e } \lambda_{f_{t}, z}^{s-1}(0)<\lambda_{f_{0}, z}^{s-1}(0) \\
\vdots \\
\text { ou } \lambda_{f_{t}, z}^{s}(0)=\lambda_{f_{0}, z}^{s}(0), \lambda_{f_{t}, z}^{s-1}(0)=\lambda_{f_{0}, z}^{s-1}(0), \ldots, \lambda_{f_{t}, z}^{1}(0)=\lambda_{f_{0}, z}^{1}(0) \\
e \lambda_{f_{t}, z}^{0}(0) \leq \lambda_{f_{0}, z}^{0}(0) .
\end{gathered}
$$

Demonstração. Aplicando o Teorema 4.16 indutivamente, como no Corolário 4.6, nós achamos que se $0 \ll j_{0} \ll j_{1} \ll \ldots \ll j_{s-1}$, então, para todo $t$ pequeno, $f_{t}+z_{0}^{j_{0}}+\ldots+z_{s-1}^{j_{s-1}}$, tem uma singularidade isolada na origem, e esse número de Milnor é dado por

$$
\begin{aligned}
\mu\left(f_{t}+z_{0}^{j_{0}}+\ldots+z_{s-1}^{j_{s-1}}\right) & =\lambda_{f_{t}, z}^{0}(0)+\left(j_{0}-1\right) \lambda_{f_{t}, z}^{1}(0)+\left(j_{0}-1\right)\left(j_{1}-1\right) \lambda_{f_{t}, z}^{2}(0)+\ldots \\
& +\left(j_{0}-1\right)\left(j_{1}-1\right) \ldots\left(j_{s-1}-1\right) \lambda_{f_{t}, z}^{s}(0) .
\end{aligned}
$$

Como o número de Milnor é semicontínuo superiormente, a conclusão segue. 


\section{Referências Bibliográficas}

[1] Atiyah, M. F. and Macdonald, I. G., Introduction to commutative algebra, Addison-Wesley Publishing Company, Inc., Massachusetts (1969).

[2] Carneiro, M. J. D. and Soares, M. G., Introdução à topologia de singularidades complexas, Instituto de Matemática Pura e Aplicada (IMPA), $15^{\circ}$ Colóquio Brasileiro de Matemática, Rio de Janeiro, (1985).

[3] Eisenbud, D. and Harris, J., Schemes: the language of modern algebraic geometry, Wadsworth \& Brooks/Cole Advanced Books \& Software, Pacific Grove, CA, (1992).

[4] Fulton, W., Intersection theory, Springer-Verlag, (1984).

[5] Gaffney, T., Polar multiplicities and equisingularity of map germs, Topology, vol. 32, no. 1 (1993), p. 185-223.

[6] Greuel, G.-M., A singular introduction to commutative algebra, Springer-Verlag, (2002). http: www.singular.uni-kl.de

[7] Gibson, C. G., Singular points of smooth mappings, Pitman Publishing - London (1979).

[8] Hamm, H. and Lê, D. T., Un Théorème de Zariski du type de Lefschetz, Ann. Sci. L'Ecole Norm. Sup. 6 (1973), p. 317-366.

[9] Kato, M. and Matsumoto, Y., On the connectivity of the Milnor fibre of a holomorphic function at a critical point, Proc. of 1973 Tokyo manifolds conf. (1973), p. 131-136.

[10] Lê, D. T. and Ramanujam, C.P., The invariance of Milnor's numbers implies the invariance of the topological type, Amer. Journ. Math. 98 (1976), p. 67-78. 
[11] Lê, D. T. and Saito, K., La constance du nombre de Milnor donne des bonnes stratifications, C.R. Acad. Sci. 277 (1973), p. 793-795.

[12] Lê, D. T., Calcul du nombre de cycles évanouissants d'une hypersurface complexe, Ann. Inst. Fourier, Grenoble 23 (1973), p. 261-270.

[13] Massey, D., The Lê-Ramanujam problem for hypersurfaces with one-dimensional singular sets, Math. Annalen 282 (1988), p. 33-49.

[14] Massey, D., Lê cycles an hypersurface singularities, Springer Lecture Notes in Mathematics 1615, (1995).

[15] Massey, D., Numerical invariants of perverse sheaves, Duke Math. J. 73 (1994), p. 307369.

[16] Milnor, J., Singular Points of Complex Hypersurfaces, Annals of Math.Studies 61, Princeton (1968).

[17] Milnor, J. and Orlik, P., Isolated singularities defined by weighted homogeneous polynomials, Topology 9 (1970), p. 385-393.

[18] Oka, M., On the homotopy type of hypersurfaces defined by weighted homogeneous polynomials, Topology 12 (1973), p. 19-32.

[19] Sakamoto, K., The Seifert matrices of Milnor fibering defined by holomorphic functions, J. Math. soc. Japan 26 (4) (1974), p. 714-721.

[20] Sebastiani, M. and Thom, R., Un résultat sur la monodromie, Invent. Math. 13 (1971), p. 90-96.

[21] Siu, Y. T. and Trautmann, G., Gap-sheaves and extension of coherent analytic subsheaves, Springer Lecture Notes in Mathematics 172, (1971). 